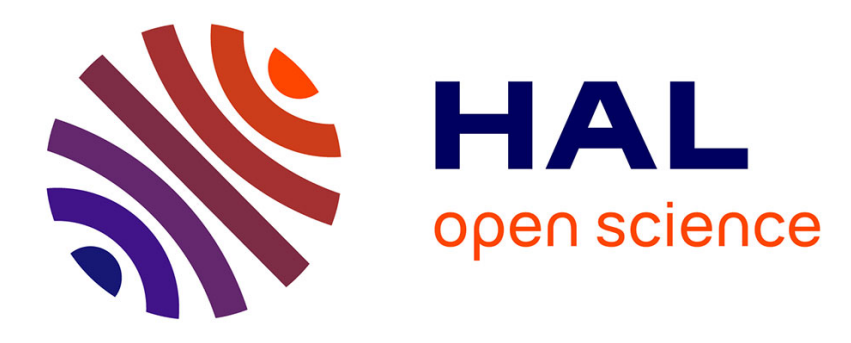

\title{
Diodes électroluminescentes organiques : quatre technologies différentes
}

Joëlle Rault-Berthelot, Cyril Poriel

\section{To cite this version:}

Joëlle Rault-Berthelot, Cyril Poriel. Diodes électroluminescentes organiques: quatre technologies différentes. Les Techniques de l'Ingenieur, 2021, pp.E6507. 10.51257/a-v1-e6507 . hal-03388539

HAL Id: hal-03388539

https://hal-univ-rennes1.archives-ouvertes.fr/hal-03388539

Submitted on 26 Oct 2021

HAL is a multi-disciplinary open access archive for the deposit and dissemination of scientific research documents, whether they are published or not. The documents may come from teaching and research institutions in France or abroad, or from public or private research centers.
L'archive ouverte pluridisciplinaire HAL, est destinée au dépôt et à la diffusion de documents scientifiques de niveau recherche, publiés ou non, émanant des établissements d'enseignement et de recherche français ou étrangers, des laboratoires publics ou privés. 


\title{
Diodes électroluminescentes organiques : quatre technologies différentes \\ Organic light-emittig diodes : four different technologies
} par Joëlle RAULT-BERTHELOT

\author{
Directrice de recherche au CNRS \\ Université de Rennes, CNRS, ISCR-UMR CNRS 6226, F-35000 Rennes, France \\ Fonction :
}

et Cyril PORIEL

Directeur de recherche au CNRS

Université de Rennes, CNRS, ISCR-UMR CNRS 6226, F-35000 Rennes, France

\begin{abstract}
Résumé
Les diodes organiques électroluminescentes (OLEDs) sont des dispositifs électroniques capables d'émettre de la lumière sous l'effet d'un champ électrique. L'architecture de base d'une OLED consiste en un film mince émetteur de lumière (EML) déposé entre deux électrodes. Aujourd'hui, il existe 4 générations d'OLEDs différenciées par le processus photo-physique conduisant à l'émission de la lumière : I'OLED «fluorescente», l'OLED «phosphorescente», l'OLED «TADF» et I'OLED «hyperfluorescente». Cet article présente les 4 générations d'OLEDs pour lesquelles le mécanisme d'émission de lumière sera discuté à la lumière d'exemples sélectionnés dans la littérature.
\end{abstract}

\begin{abstract}
An organic light-emitting diode is an electronic device able to emit light in response to an electric current. The basic structure of an OLED consists in a thin film of organic emitting material (EML) deposited between two electrodes. Today, there are 4 generations of OLEDs depending on the photo-physical process involved in the light emission: the "fluorescent OLED", the "phosphorescent OLED", the "TADF OLED" and the the "hyperfluorescent OLED". This article presents the four types of OLEDs for which, the mechanism of light emission will be discussed at the light of selected examples judiciously chosen in literature.
\end{abstract}

\section{Mots-clés ( 3-4) :}

Electronique organique, OLED, fluorescence, phosphorescence, fluorescence retardée activée thermiquement, hyperfluorescence

\section{Keywords ( 3-4) :}

Organic electronic, OLED, fluorescence, phosphorescence, thermally activated delayed fluorescence, hyperfluorescence 


\section{Table des matières}

1 Contexte

2 Les OLEDs fluorescentes __ 5

2.1 Principe de fonctionnement d'une OLED fluorescente

2.2 Exemples d'OLEDs fluorescentes _ 5

3 Les OLEDs phosphorescentes__ 8

3.1 Principe de fonctionnement d'une OLED phosphorescente

3.2 Emission bleue des OLEDs phosphorescentes ___

3.3 Emission verte des OLEDs phosphorescentes ___ 11

3.4 Emission jaune/orange des OLEDs phosphorescentes ___ 13

3.5 Emission rouge des OLEDs phosphorescentes

3.6 Emission blanche des OLEDS phosphorescentes __ 16

4 Les OLEDs à fluorescence retardée activée thermiquement___ 18

4.1 Principe de fonctionnement d'une OLED TADF _ 18

4.2 Emission bleue des OLEDs TADF

4.3 Emission verte des OLEDs TADF

4.4 Emission jaune/orange des OLEDs TADF _ 22

4.5 Emission rouge des OLEDs TADF _ 23

4.6 Emission blanche des OLEDs TADF

5 Les OLEDs hyperfluorescentes__ 26

5.1 Principe de fonctionnement d'une OLED hyperfluorescente

5.2 Exemples d'OLEDs hyperfluorescentes _ 27

6. Conclusion générale _ 29

Glossaire —31

Sigles__ 31

\section{Contexte}

Depuis la découverte de l'électroluminescence organique dans les années 50,[1] puis les travaux pionniers sur l'émission de lumière à partir de couche épaisses de cristaux de molécules aromatiques (anthracène, pyrène, tétracène) des années 60,[2] il a fallu attendre 1987, la démonstration par Tang et Van Slyke (société Eastman-Kodak) de la première diode organique électroluminescente ou OLED (pour Organic Light-Emitting Diode).[3] Depuis lors, les OLEDs ont été le sujet d'intenses recherches de la part des chimistes, des physiciens et des électroniciens.[4]

Si l'on se réfère à l'article paru sur le site web C|NET en novembre 2020 qui en fait l'inventaire,[5] le marché compte désormais de nombreux téléviseurs OLED. La technologie OLED permet des niveaux de contraste quasiment infinis, des couleurs d'une grande vivacité, des angles de vision extrêmement larges, tandis que l'absence de rétro-éclairage permet de nouveaux designs plus fins, et même des téléviseurs à écran enroulable. Enfin, la société Xiaomi a dévoilé en août 2020 un téléviseur OLED entièrement transparent, le Mi TV LUX, réservé pour le moment au marché chinois.[6] Contrairement 
aux téléviseurs traditionnels, le Mi TV LUX n'est pas doté d'un panneau arrière et il est possible de voir à travers son écran lorsque celui-ci est éteint. Le constructeur a réuni tous les composants dans un socle circulaire qui fait également office de haut-parleur. On retrouve aussi les OLEDs dans des systèmes d'affichage ou d'éclairage.[7-10] Les premiers téléphones pliables ont été commercialisés en 2019 par Samsung[11] et constituent une véritable révolution dans le domaine de l'électronique souple. Samsung annonce aujourd'hui avoir encore réduit l'angle de courbure de ses écrans pliables à $1.4 \mathrm{~mm} .[12]$

Si les premières OLEDs,[3] étaient basées sur des molécules ou des polymères fluorescents[13-16] avec des rendements limités à $25 \%$ du fait de la perte d'énergie par désactivations non radiatives, elles ont peu à peu cédé la place aux OLEDs à émetteur phosphorescent, nommées PhOLEDs[17-19] (pour Phosphorescent Organic Light-Emitting Diodes), découvertes en 1998,[20] permettant théoriquement de quadrupler les performances maximales des dispositifs. En 2009,[21] un nouveau mécanisme d'émission a été mis en évidence : la fluorescence retardée activée thermiquement TADF[22-24] (pour Thermally Activated Delayed Fluorescence). Cette troisième génération de diode TADF-OLED permet de s'affranchir des émetteurs phosphorescents organométalliques souvent à base de Platine ou d'Iridium tout en gardant des rendements d'émission très élevés liés à une conversion totale des excitons singulet et triplet formés. Plus récemment, une quatrième génération d'OLED, les OLEDs hyperfluorescentes [25] a vu le jour, ces nouveaux dispositifs combinent l'intérêt du TADF et celui de la fluorescence et sont annoncés comme hautement efficaces, de faible coût et d'émission de couleur de qualité supérieure.

Les performances d'un dispositif OLED en terme de couleur d'émission et d'efficacité de la conversion énergétique dépendent notamment de la nature des matériaux qui constituent la couche émissive (molécule ou macromolécule), de la structure de la diode (monocouche, multicouche) et du mode de fabrication de la diode (évaporation sous vide, dépôt des couches en solution,...). Toutefois, les performances d'une OLED sont aussi grandement dépendantes du principe de fonctionnement de la diode et particulièrement de la nature des transferts énergétiques impliqués dans le dispositif. Cet article décrit, pour chaque génération d'OLED, son principe de fonctionnement, ses limites et quelques performances caractéristiques rapportées à ce jour dans la littérature afin d'éclairer le lecteur sur l'évolution de cette technologie.

Une OLED « monocouche ", dispositif d'émission de lumière le plus simple, se présente sous la forme d'un film semi-conducteur organique (SCO) émetteur de lumière pris en sandwich entre deux électrodes (une anode transparente et une cathode métallique) (figure 1a).[3, 26]

L'électroluminescence (EL) est provoquée par l'injection dans la couche organique émettrice (EML pour Emitting Layer) de trous venant de l'anode et d'électrons venant de la cathode, du transport de ces charges dans le matériau autorisant leur recombinaison en paires électron-trou («excitons») puis de la relaxation de ces excitons avec émission de lumière (figure $1 \mathrm{~b}$ ).

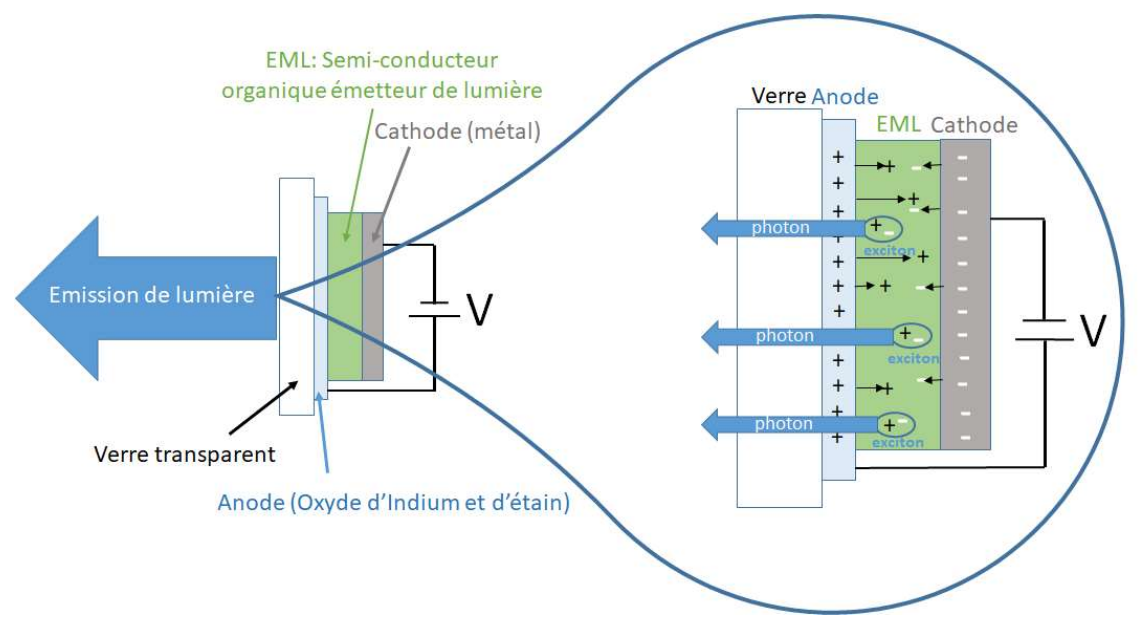

Figure 1. Structure et principe général de l'électroluminescence d'une OLED monocouche 
La lumière émise par l'OLED, directement liée à la nature de la couche organique et plus précisément de l'écart entre l'orbitale moléculaire la plus haute occupée (HOMO pour Highest Occupied Molecular Orbital) et l'orbitale moléculaire la plus basse vacante (LUMO pour Lowest Unoccupied Molecular Orbital) de l'émetteur fluorescent utilisé, est déterminée selon un système de référence colorimétrique de la Commission Internationale de l'Eclairage (CIE) au moyen d'un diagramme de chromaticité (voir figure 15).[27]

Afin d'améliorer l'injection, le transport et la recombinaison des charges dans la couche émissive, des dispositifs dits " multicouches » intégrant des couches additionnelles de matériaux organiques à propriétés dédiées (aidant à l'injection, au transport et/ou au blocage des charges) sont utilisés. Ainsi il existe deux grands types d'architectures d'OLEDs, quel que soit le mécanisme d'émission : les OLEDs « monocouches » uniquement constituées des électrodes et de la couche émissive et les OLEDs « multicouches » possédant de part et d'autre de la couche émissive un nombre variable de couches additionnelles. Les dispositifs " multicouches " sont généralement plus performants mais les dernières années, d'énormes progrès ont été faits dans le domaine des OLEDs « monocouches " qui, de par leur simplicité, sont très intéressantes pour diminuer le coût énergétique et de production.

Les performances d'une OLED peuvent être caractérisées par la mesure de sa signature couranttension-luminance (courbe I-V-L), obtenue par l'application d'une tension croissante aux bornes du dispositif et du relevé point par point du courant traversant la diode et de la lumière produite. De ces enregistrements, on obtient la tension de seuil en $V_{\text {olt }}\left(\mathrm{V}_{\text {on }}\right.$ : tension minimale qu'il faut appliquer à la diode pour qu'elle commence à éclairer), la luminance en $\mathrm{cd} / \mathrm{m}^{2}$, le rendement de courant (CE pour Current Efficiency) en cd/A, l'efficacité énergétiques (PE pour Power Efficiency) en Im/W, le rendement quantique externe EQE (EQE pour External Quantum Efficiency) en \% qui est le rapport entre le nombre d'électrons injectés et le nombre de photons émis, la longueur d'onde maximale d'émission obtenue par le spectre d'électroluminescence (spectre d'EL) et les coordonnées chromatiques.

Ces éléments ont été déjà discutés par différents auteurs dans des articles publiés dans Techniques de l'Ingénieur de Destruel et Jolinat en 2004[28], Fery et Le Roy en 2005[29], Le Barny en 2006[30, 31] et Jolinat en 2011[32] et 2016[33], Sebahi, Cornil et Viville en 2011[34], Forget et Chenais en 2014[35], ou par nous-mêmes en 2013[36], 2014[37] et 2015[38] pour les OLEDs fluorescentes.

Toutefois, les performances d'une OLED sont aussi grandement dépendantes des processus photophysiques impliqués dans l'émission de la lumière. C'est même aujourd'hui le paramètre le plus important car c'est lui qui limite théoriquement les performances de l'OLED. Si dans sa genèse, le terme OLED faisait référence aux OLEDs fluorescentes[13-15, 39, 40] depuis quelques années les OLEDs phosphorescentes,[17-19, 41, 42] TADF[22, 24, 43] et hyperfluorescentes[44, 45] les ont supplantées. C'est à l'évolution de cette technologie et à ses origines que s'intéresse le présent article.

\section{À retenir}

- Une OLED est un dispositif électronique émettant de la lumière sous l'effet d'un champ électrique.

- L'électroluminescence est provoquée par l'injection de charges dans une couche organique, leur recombinaison en excitons et l'émission de lumière par désexcitation radiative des excitons.

- La lumière émise par la diode est liée à la nature de la couche organique émissive

- Les performances d'une OLED dépendent du processus photophysique entrainant l'émission de lumière (fluorescence, phosphorescence,...). 


\section{Les OLEDs fluorescentes}

Ce sont les OLEDs fluorescentes qui sont à l'origine du développement de cette nouvelle technologie d'affichage et d'éclairage.

\subsection{Principe de fonctionnement d'une OLED fluorescente}

Les OLEDs fluorescentes sont basées sur des SCO fluorescents (molécules ou macromolécules). Lors de la mise sous tension du dispositif, des électrons et des trous provenant des électrodes (Figure 1b) sont injectés dans le SCO, ils se combinent pour former des paires électron-trou nommés excitons dans un rapport déterminé par la statistique de spin de $25 \%$ d'excitons singulets et $75 \%$ d'excitons triplets (Figure 2). [20,46] Les molécules organiques n'étant pas dans leur majorité émetteur via le niveau $T_{1}$ à température ambiante, les excitons triplets sont perdus par désexcitation non radiative (retour de $T_{1}$ vers $S_{0}$ sans émission de lumière) et seuls les excitons singulets se désactivent en émettant de la lumière (retour de $S_{1}$ vers $S_{0}$ ).

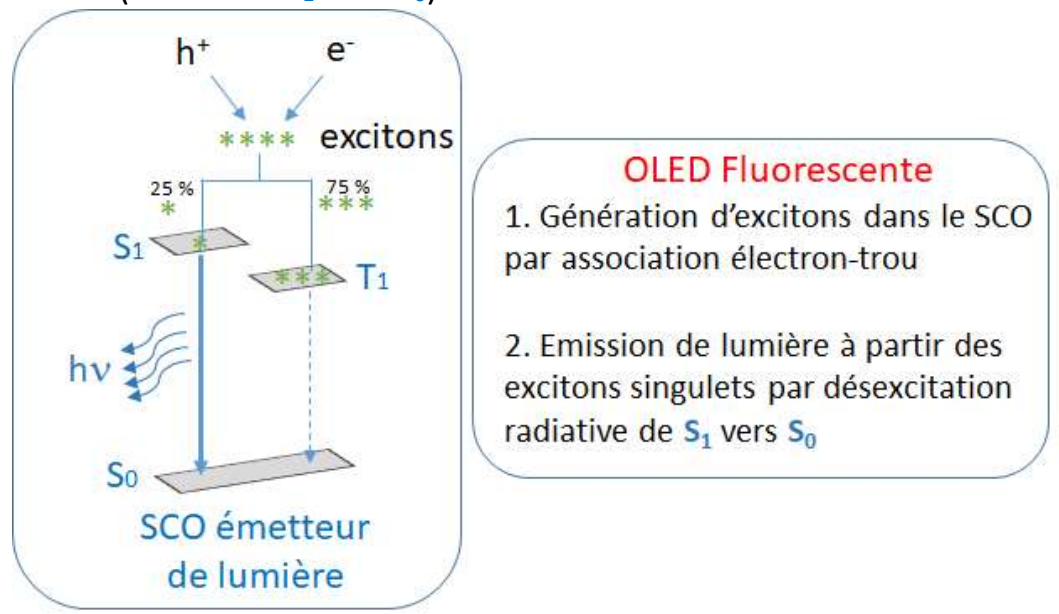

Figure 2. Principe de fonctionnement d'une OLED fluorescente

Le rendement d'efficacité quantique interne maximum IQE (IQE pour Internal Quantum Efficiency) est donc limité à $25 \%$ du fait de la perte d'énergie par désactivation non radiative des excitons triplets, entrainant des EQE rarement supérieurs à 5-7.5\% selon l'efficacité du découplage de la lumière dans le dispositif ( $\eta_{\text {out }} \sim 0.2-0.3$ ).[47]

Pour qu'un SCO puisse être utilisé comme émetteur dans la couche active d'une OLED fluorescente, il faut qu'il réponde à plusieurs critères :

(i) un caractère bipolaire pour pouvoir être oxydé et réduit facilement aux deux électrodes,

(ii) des niveaux HOMO/LUMO adaptés au niveau de Fermi des électrodes pour faciliter l'injection des charges dans le dispositif,

(iii) des mobilités d'électrons et de trous équilibrées pour optimiser la recombinaison des paires électron-trous et la formation des excitons dans la couche active,

(iv) de bonnes stabilités thermiques (haute température de décomposition et haute température de transition vitreuse) et morphologiques pour ne pas se décomposer ou évoluer lorsque la diode est en fonctionnement,

(v) un rendement de fluorescence à l'état solide le plus élevé possible.

Enfin, dans le cas idéal, la couleur de la lumière émise par l'OLED fluorescente sera celle émise par le SCO à l'état solide. II existe aujourd'hui un nombre important d'OLEDs fluorescentes émettant dans tout le spectre visible (bleu à rouge), quelques exemples caractéristiques sont présentés ci-dessous.

\subsection{Exemples d'OLEDs fluorescentes}

Les performances reportées dans le tableau 1 sont celles de diodes fluorescentes d'architecture mono- ou multicouche. Il est évident que pour pouvoir atteindre des dispositifs commercialisables, on recherchera des dispositifs d'architecture simple et le plus efficace possible. Dans cette technologie, 
le dispositif « multicouche " (constitué d'assemblage de couches organiques assurant l'injection, le transport, le blocage et la recombinaison des charges) est de loin le plus étudié mais le dispositif " monocouche" (constitué uniquement des électrodes et de la couche émissive), tend à combler son retard en terme de performance et apparait beaucoup plus simple et moins énergivore.[26] Les structures chimiques des émetteurs utilisés dans la couche active de ces diodes sont représentées figure 3 .

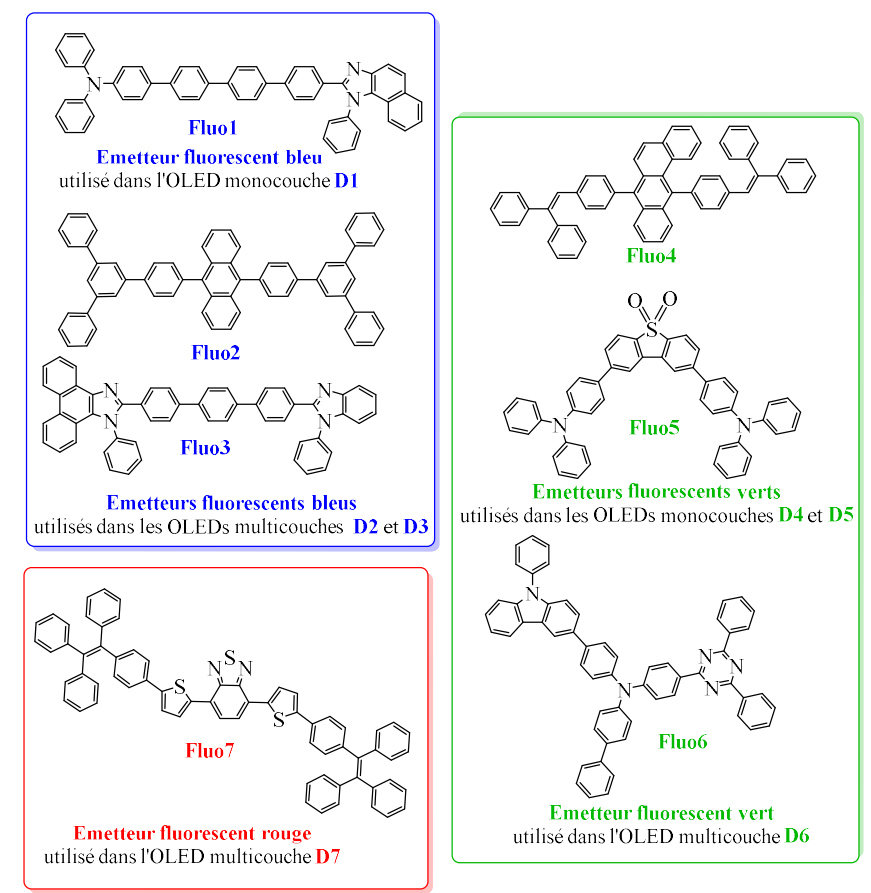

Figure 3. Exemples de SCOs fluorescents utilisés en OLED fluorescentes

Le dispositif $D 1$ possède une couche émissive composée du fluorophore Fluo1 construit sur un design Donneur-système $\pi$-Accepteur ( $D-\pi-A)$ composé d'un terphényl en système $\pi$ central, $d^{\prime}$ une triphénylamine comme groupe donneur d'électrons et d'un dérivé d'imidazole comme groupe accepteur d'électrons. [48] Dans ce dispositif monocouche, l'équilibre du transport des charges et le bon alignement des niveaux HOMO/LUMO (-5.18/-2.35 eV) de Fluo1 aux niveaux de Fermi des électrodes conduisent à une émission de la diode dès $2.6 \mathrm{~V}$ et un EQE de $5.12 \%$ indiquant l'excellence de l'injection et du transport des charges, de leurs combinaisons en exciton et du processus de désexcitation de ces excitons. On notera que l'anode de D1 est une couche d'ITO recouverte d'une couche de 1,4,5,8,9,11-hexatriphenylene-hexacarbonitrile (HAT-CN) pour aider à l'injection des trous (HIL pour Hole Injecting Layer) et la cathode est en aluminium (Al) recouvert d'une fine couche de fluorure de lithium (LiF) pour aider à l'injection des électrons (EIL pour Electron Injecting Layer).

\begin{tabular}{|c|c|c|c|c|c|c|c|c|}
\hline & Architecture du dispositif & $\begin{array}{l}V_{\text {on }} \\
(V)\end{array}$ & $\begin{array}{l}\mathrm{EQE}_{\max } \\
(\%)\end{array}$ & $\begin{array}{l}\mathrm{CE}_{\max } \\
(\mathrm{cd} / \mathrm{A})\end{array}$ & $\begin{array}{c}P E_{\max } \\
(\mathrm{Im} / \\
\mathrm{W})\end{array}$ & $\begin{array}{l}\lambda_{\max } \\
(\mathrm{nm})\end{array}$ & $\begin{array}{c}\mathrm{CIE} \\
(\mathrm{x}, \mathrm{y})\end{array}$ & Réf. \\
\hline \multicolumn{9}{|c|}{ Dispositif monocouche Bleu } \\
\hline D1 & ITO/HAT-CN(5nm)/Fluo1(80nm)/LiF(1nm)/Al & 2.6 & 5.12 & 4.95 & 4.96 & 450 & $0.15,0.11$ & [48] \\
\hline \multicolumn{9}{|c|}{ Dispositifs multicouches Bleu } \\
\hline D2 & $\begin{array}{l}\text { ITO/2-TNATA(60nm)/NPB(15nm)/Fluo2(30nm) } \\
\text { /Alq3(30nm)/LiF }(1 \mathrm{~nm}) / \mathrm{Al}(200 \mathrm{~nm})\end{array}$ & 6.75 & 7.18 & 3.64 & 1.87 & 444 & $0.16,0.09$ & {$[49,50]$} \\
\hline D3 & $\begin{array}{l}\text { (ITO)/HAT-CN(5nm)/NPB }(70 \mathrm{~nm}) / \mathrm{TCTA}(5 \mathrm{~nm}) / \\
\text { Fluo3 }(25 \mathrm{~nm}) / \mathrm{TPBI}(40 \mathrm{~nm}) / \mathrm{LiF}(1 \mathrm{~nm}) / \mathrm{Al}(150 \mathrm{~nm})\end{array}$ & 2.6 & 7.11 & 6.78 & 7.1 & 452 & $0.15,0.13$ & [51] \\
\hline \multicolumn{9}{|c|}{ Dispositifs monocouche Vert } \\
\hline D4 & $\begin{array}{l}\text { ITO/Fluo4(90nm)/Li } 3 \mathrm{~N}(1 \mathrm{~nm}) / \mathrm{Mg}: \mathrm{Ag}(150 \mathrm{~nm}) / \\
\mathrm{Ag}(50 \mathrm{~nm})\end{array}$ & 3.1 & 3.2 & 11.4 & & $\begin{array}{l}528 \\
560\end{array}$ & - & [52] \\
\hline D5 & ITO/Fluo5(80nm)/LiF(1nm)/Al(150nm) & 2.2 & 3.1 & 7.7 & 7.2 & 496 & $0.16,0.44$ & [53] \\
\hline \multicolumn{9}{|c|}{ Dispositif multicouche Vert } \\
\hline D6 & $\begin{array}{l}\text { ITO/HAT-CN(20nm)/TAPC(30nm)/Fluo6/ } \\
\text { BPhen/LiF/AI }\end{array}$ & 3.1 & 4.4 & - & 11.8 & 514 & $0.26,0.56$ & [54] \\
\hline \multicolumn{9}{|c|}{ Dispositif multicouche Rouge } \\
\hline D7 & $\begin{array}{l}\text { ITO/NPB(60nm)/Fluo7(20nm)/TPBI(40nm)/LiF } \\
(1 \mathrm{~nm}) / \mathrm{Al}(100 \mathrm{~nm})\end{array}$ & 4.2 & 3.7 & 2.4 & & 650 & $0.67,0.32$ & [55] \\
\hline
\end{tabular}

Tableau 1. Exemples de performances de dispositifs OLEDs fluorescentes 
Dans les dispositifs D2 et D3, utilisant respectivement l'émetteur fluorescent Fluo2 [49, 50] et Fluo3,[51] deux et trois couches supplémentaires ont été ajoutées de part et d'autre de l'EML. Dans D2, côté anode, 2-TNATA $\left(4,4^{\prime}, 4^{\prime \prime}\right.$-tris( $N$-(2-naphtyl)- $N$-phenylamino)triphenylamine) est l'HIL et NPB ( $N, N^{\prime}$-bis(naphtalen-1-yl)- $N, N^{\prime}$-bis(phenyl)benzidine) aide au transport des trous (HTL pour Hole Transporting Layer ), côté cathode, Alq ${ }_{3}$ est une couche aidant au transport des électrons (ETL pour Electron Transporting Layer).

Dans D3, NPB est l'HTL, le tris(4-carbazoyl-9-ylphényl)amine (TCTA) est une couche bloqueuse d'électrons (EBL pour Electron Blocking Layer) qui aide aussi au transport des trous, enfin, une couche de 2,2',2"-(1,3,5-Benzinetriyl)-tris(1-phényl-1-H-benzimidazole) (TPBI) joue le rôle de transporteur d'électrons (ETL pour Electron Transporting Layer) et de bloqueur de trous (HBL pour Hole Blocking Layer).

D2 et D3 ont des EQE $E_{\max }$ supérieurs à 7\% montrant l'effet positif de l'addition de couches intermédiaires sur la performance des dispositifs. Pour D2, l'émetteur Fluo2 est un composé purement hydrocarboné PHC (PHC pour Pure HydroCarbon, un PHC ne comporte pas d'hétéroatome et est uniquement constitué d'atomes de carbone et d'hydrogène, pour des exemples récents de $\mathrm{PHC}$ en EO voir [56-60]) rendant plus difficile l'oxydation de la molécule comparée à celle de Fluo1 (HOMO: 5.18/-5.54 eV pour Fluo1/Fluo2). De ce fait, le $V_{\text {on }}$ de D2 (6.75 V) est beaucoup plus élevée que celui de D1 (2.6 eV).

Dans D3, l'émetteur Fluo3 a, comme Fluo1, une architecture D- $\pi$-A. Les groupes donneur et accepteur d'électrons sont tous les deux des dérivés d'imidazole, le niveau HOMO de Fluo3 $(-5.46 \mathrm{eV})$ est plus profond que celui de Fluo1 $(-5.18 \mathrm{eV})$ alors que les niveaux LUMO sont similaires (-2.34 / -2.35 eV pour Fluo3 / Fluo1). D'autre part, du fait de l'ajout de couches intermédiaires entre Fluo3 et l'anode ITO/HAT-CN, l'injection des trous est facilitée et le $V_{\text {on }}$ de D3 est identique à celui de D1 (2.6 V).

D1 et D3 ont un spectre d'EL centré à $450 \mathrm{~nm}$ alors que celui de D2 est décalé vers les basses longueurs d'onde entrainant une diode présentant des coordonnées chromatiques ( $\mathrm{CIE}(\mathrm{x}, \mathrm{y}): 0.16$, 0.09) possédant une valeur de y inférieur à 0.1 signant pour D2 une émission bleue plus intense que pour D1 $(0.15,0.11)$ et D3 $(0.15,0.13)$.

Les OLEDs émissives de lumière bleue, couleur plus difficile à obtenir que les lumières vertes ou rouges, ont été beaucoup plus étudiées et décrites dans la littérature que ces dernières.[26, 61-63] Les OLEDs vertes et rouges les plus efficaces ont paradoxalement des performances inférieures à celles des meilleures diodes bleues. Cela peut être interprété par le fait que les OLEDs rouge et verte ont plus vite atteint des performances intéressantes, nécessitant moins de travaux dans ces domaines. L'OLED D4 est un dispositif monocouche à base du PHC Fluo4. Ce fluorophore émet dans le bleu $\left(\lambda_{\max }: 443 \mathrm{~nm}\right)$ en solution diluée.[52] L'augmentation de la concentration de la solution entraîne l'apparition d'une bande d'émission dans le vert $\left(\lambda_{\max }: 514 \mathrm{~nm}\right)$ attribuée par les auteurs à une émission excimérique provenant de l'émission d'excimères intermoléculaires formés par interaction à l'état excité entre deux unités monomères. Les deux bandes d'émission (bleue et verte) sont présentes dans le spectre de fluorescence de Fluo4 à l'état solide. Le spectre d'EL de l'OLED D4 ne présente que l'émission dans le vert indiquant que l'émission de lumière provient uniquement des excimères (pour des travaux sur les OLEDs à base d'excimères vous pouvez consulter la référence [36]). Bien que Fluo 4 ne possède pas de caractère ambipolaire, la tension de seuil de D4 est relativement faible $(3.1 \mathrm{eV})$ et l'EQE $E_{\max }$ atteint $3.2 \%$.

Dans I'OLED monocouche D5, [53] le fluorophore Fluo5 présente une architecture Donneur-Système $\pi$-Accepteur-Système $\pi$-Donneur (D- $\pi-A-\pi-D)$, des niveaux HOMO/LUMO plus bas en énergie (-5.35/ $-2.61 \mathrm{eV})$ que ceux de Fluo4 $(-5.74 /-2.67 \mathrm{eV})$. D'autre part, la diode émet à très bas potentiel dès 2.2 $\mathrm{eV}$ et son EQE atteint $3.1 \%$.

La diode multicouche verte D6 [54] a comme émetteur, Fluo6, un dérivé d'architecture DonneurDonneur-Accepteur (D-D-A) dans lequel les unités triphénylamine et carbazole aident à l'injection et au transport des trous à l'anode tandis que l'unité triazine aide à l'injection et au transport des électrons. On notera dans cette diode multicouche, la présence d'une couche de di-[4-(N,N-di-p-tolylamino)-phényl]cyclohexane (TAPC) entre l'anode et l'EML et d'une couche de 4,7-diphényl-1,10- 
phénanthroline (BPhen) entre l'EML et la cathode. Ces deux couches jouent respectivement le rôle d'HTL (TAPC) et d'EIL, ETL et HBL (BPhen). Les niveaux HOMO/LUMO de Fluo6 (-5.35/-2.61 eV) montrent un SCO facile à oxyder et à réduire. De ce fait, I'OLED D6 s'allume dès $3.1 \mathrm{~V}$ et atteint un $E E_{\max }$ de $4.4 \%$ pour une émission verte de coordonnées $\operatorname{CIE}(0.26,0.56)$.

La diode $\mathrm{D} 7$ basée sur le fluorophore Fluo7 émet une lumière rouge dès $4.2 \mathrm{~V}$ avec un $\mathrm{EQE}_{\max }$ de 3.7 $\%$.[55] Fluo7 présente, à l'état solide, un spectre de fluorescence centré à $646 \mathrm{~nm}$ similaire au spectre d'EL de l'OLED D7. On notera dans cette diode multicouche la présence entre l'anode et I'EML de NPB (HIL et HTL) et entre l'EML et la cathode de TPBI (ETL).

Conclusion : Les OLEDs fluorescentes dont l'EQE $E_{\max }$ est limité théoriquement à $5-7.5 \%$ selon l'efficacité du découplage de la lumière $\left(\eta_{\text {out }} \sim 0.2-0.3\right)[47,48]$ sont aujourd'hui moins étudiées et les quelques performances présentées ci-dessus pour les émissions rouges, vertes ou bleues restent modestes. La recherche sur les fluorescents émetteurs de lumière bleue reste malgré tout active, la lumière bleue constituant toujours un réel challenge en électronique organique. Les OLEDs fluorescentes présentent cependant l'avantage d'utiliser des molécules simples et la technologie est bien en place. Les seconde et troisième générations d'OLED vont permettre d'atteindre des dispositifs beaucoup plus performants.

\section{À retenir}

- Dans une OLED fluorescente :

- la couche organique active émet par fluorescence.

- I'IQE est au maximum de $25 \%$ ce qui entraîne un EQE de l'ordre de 5 à $7.5 \%$ selon l'efficacité du découplage de la lumière.

- Pour améliorer ses performances, on ajoute de part et d'autre de la couche émissive des couches de composés aidant à l'injection, au transport et au blocage des charges afin de maximiser leur recombinaison dans le cœur de la couche active.

\section{Les OLEDs phosphorescentes}

En 1998, l'équipe de Forrest (Princeton University USA) annonce pouvoir récupérer $100 \%$ des excitons formés dans le SCO en les transférant à un dopant phosphorescent présent en faible quantité dans ce dernier[20]. Les diodes phosphorescentes (PhOLED), dont la couche émissive est un mélange $\mathrm{SCO} /$ dopant ont alors pris peu à peu la place des OLEDs fluorescentes conduisant à une augmentation considérable des performances des dispositifs.

\subsection{Principe de fonctionnement d'une OLED phosphorescente}

Dans une PhOLED, la couche active est un SCO (servant de matrice hôte) dopé en faible quantité (en général autour de 5 à $10 \%$ en masse) par un émetteur phosphorescent (complexe d'iridium, de platine ou d'autres métaux lourds (osmium etc ...).

Lors de la mise sous tension du dispositif, comme dans le cas des OLEDs fluorescentes présentées précédemment, des électrons et des trous provenant des électrodes sont injectés dans la matrice hôte, ils se combinent pour former des paires électron-trou dans un rapport de 25/75 d'excitons singulets/excitons triplets dû à la statistique de spin (Figure 4). Dans l'EML, les excitons triplets générés dans la matrice hôte sont transférés vers le dopant phosphorescent (transfert de $T^{H_{1}} \rightarrow T^{G}$ ). De la même manière, les excitons singulets formés dans la matrice organique sont transférés vers le dopant (transfert de $S_{1} \rightarrow S^{G}{ }_{1}$ ) puis convertis en excitons triplets par croisement intersystème $\left(S_{1}^{G} \rightarrow\right.$ $T^{G}{ }_{1}$ ). Au bilan, $100 \%$ des excitons formés dans la matrice hôte se retrouvent à l'état d'excitons triplets dans le dopant et reviennent à l'état fondamental en émettant de la lumière par phosphorescence $\left(T_{1}^{G} \rightarrow S^{G}{ }_{0}\right)$. On peut noter que des excitons peuvent aussi être générés directement dans le dopant. La lumière émise est donc celle du phosphorescent dispersé dans la matrice SCO. Une des caractéristiques des OLEDs phosphorescentes par rapport aux OLEDs fluorescentes concerne la durée de vie de l'émission de lumière : de l'ordre de la microseconde pour la phosphorescence et 


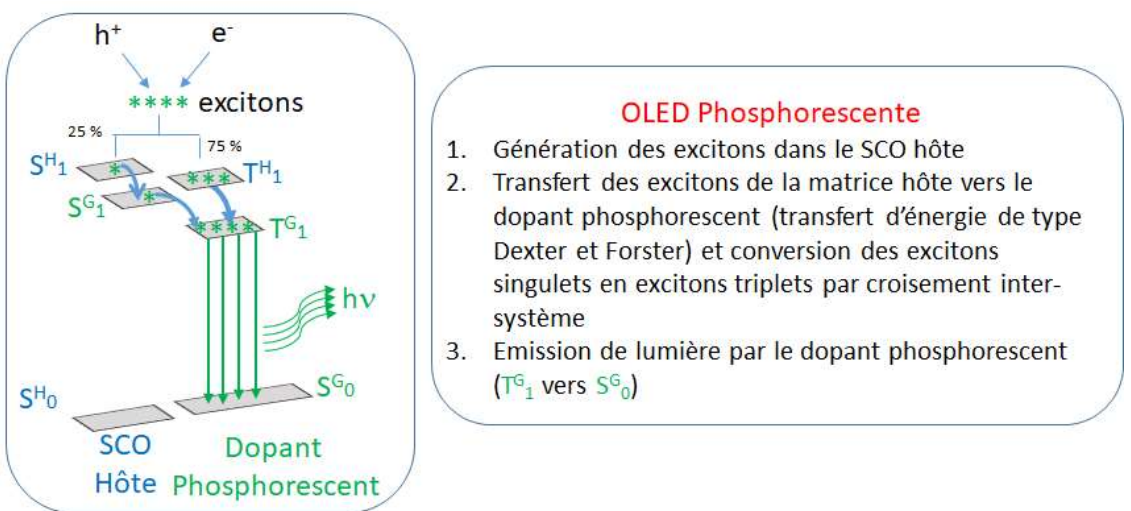

Figure 4. Principe de fonctionnement d'une OLED phosphorescente

Les propriétés requises pour qu'un SCO puisse être utilisé comme matrice hôte dans une PhOLED, sont similaires à celles décrites pour le SCO d'une OLED fluorescente pour les items (i)-(iv) (décrits pages 4/5). Toutefois, l'émission de la PhOLED provenant, non plus du SCO (qui est l'émetteur pour I'OLED et la matrice hôte pour la PhOLED), mais de son dopant, il convient de choisir le SCO en fonction des caractéristiques énergétiques de ce dernier. Ainsi, il faudra ajuster au mieux les niveaux HOMO/LUMO du SCO à ceux du dopant et il sera capital que les niveaux d'énergie $\mathrm{S}_{1}{ }_{1}$ et $\mathrm{T}^{\mathrm{H}}{ }_{1}$ soient respectivement supérieurs à ceux du dopant $S^{G}{ }_{1}$ et $T^{G}{ }_{1}$ afin que le transfert d'énergie matrice $\rightarrow$ dopant soit total. Dans ce cas uniquement, l'IQE pourra atteindre $100 \%$ (avec pour corollaire des EQE pouvant atteindre $20-30 \%$ selon l'efficacité du découplage de la lumière dans le dispositif ( $\eta_{\text {out }} \sim 0.2$ 0.3).[47]).

Grâce à ce concept, des progrès fantastiques ont pu être réalisés en termes de performances de dispositifs. Des PhOLEDs très performantes pour les principales couleurs sont présentées ci-dessous en suivant l'ordre d'évolution de leurs longueurs d'onde : bleu (450-500 nm), cyan (500-520 nm), vert (520-565 nm), jaune-orange (565-625nm) puis rouge $(625-740 \mathrm{~nm})$. Quelques PhOLEDs à dopants multiples émettrices de lumière blanche seront aussi présentées.

\subsection{Emission bleue des OLEDs phosphorescentes}

Avec une structure de dispositif monocouche très ressemblante (mêmes électrodes, mêmes HIL (poly(3,4-éthylènedioxythiophène):poly(styrène sulfonate) de sodium (PEDOT:PSS)) et EIL (LiF), mêmes dopant et taux de dopage, même épaisseur de couche active) et des matrices hôtes, $\mathbf{H 1}$ [64] et $\mathbf{H 2}$ [65], assez proches en termes de structures (Figure 5) et de propriétés électroniques, les PhOLEDs D8 et D9 ont des performances similaires (Tableau 2). Les niveaux d'énergie d'état triplet $\left(E_{T}\right)$ des matrices hôtes $\mathbf{H} \mathbf{1}$ et $\mathbf{H} \mathbf{2}$ sont mesurés à partir des spectres d'émission à $77 \mathrm{~K}$ qui permettent d'observer la contribution de phosphorescence (spectre d'émission montrant la contribution de phosphorescence de $\mathbf{H} \mathbf{2}$ encadré en Figure 5). La tension de seuil de D9 (2.5 V), un peu plus faible que celle de D8 $(3 \mathrm{~V})$, peut être expliquée par une injection et une mobilité des électrons $\left(\mu_{\mathrm{e}}\right)$ plus élevées dans H2 (LUMO:-2.46 eV / $\mu_{\mathrm{e}}: 210^{-4} \mathrm{~cm}^{2} / \mathrm{V} . \mathrm{s}$ ) que dans H1 (LUMO:-2.40 eV / $\mu_{\mathrm{e}}: 2$ 10-6 $\left.\mathrm{cm}^{2} / \mathrm{V} . \mathrm{s}\right)$. Les $\mathrm{EQE}_{\max }$ élevés, supérieurs à $17.5 \%$, signent dans les deux cas l'efficacité des processus conduisant à l'émission unique du FIrpic. Le spectre d'EL de la diode D9 est aussi présenté dans l'encadré de la figure 5 mettant en évidence l'émission unique du Flrpic, sans aucune émission de la matrice, et le total transfert d'énergie entre la matrice hôte $\mathbf{H 2}$ et le dopant FIrpic dans D9.

Les dispositifs D10-D15 sont des dispositifs multicouches à 3 (D10-D14) ou 4 (D15) couches intermédiaires additionnelles dont les EQE $E_{\max }$ dépassent tous $24.7 \%$.[56, 66, 67, 68] 

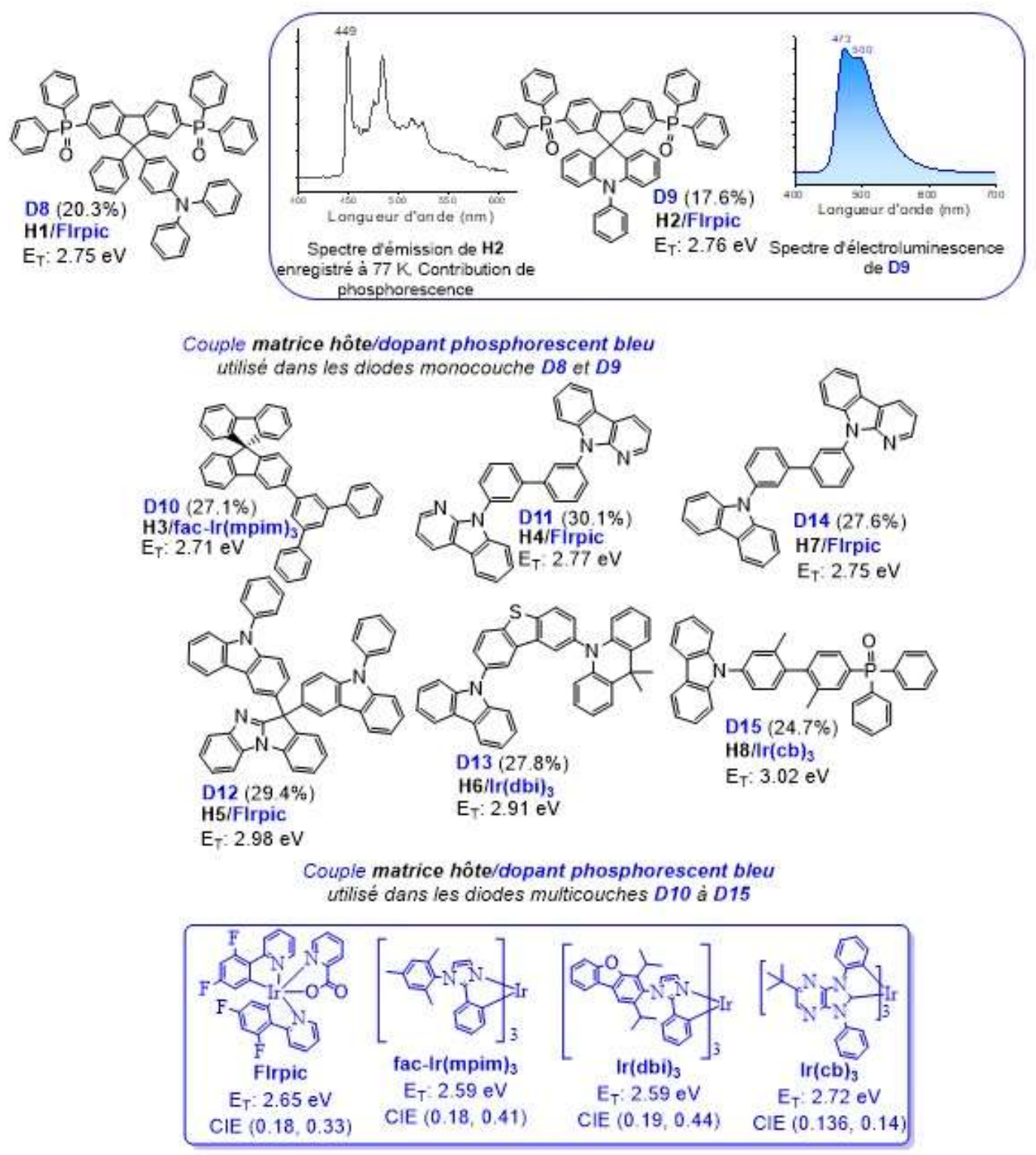

Figure 5. Exemples de couple matrice hôte/dopant phosphorescent bleu

La diode D11 ayant comme couche émissive le couple H4:FIrpic $10 \%$ et trois couches additionnelles aidant à l'injection, au transport et au blocage des porteurs de charge (TAPC et 1,3-Bis(Ncarbazolyl)benzene (mCP) entre l'anode et l'EML et diphényl[4-(triphénylsilyl)phényl]phosphine oxide (TSPO1) entre l'EML et la cathode), dépasse même les $30 \%$ d'EQE. [68] Ces performances sont très élevées.

D10 [56] utilise un dopant phosphorescent à base de phénylimidazole, fac- $\operatorname{Ir}(\text { iprpmi })_{3}$, atteint $27.1 \%$ d'EQE. Sa matrice hôte $\mathbf{H 3}$ est un PHC d'ET de $2.82 \mathrm{eV}$ bien adapté à celle du fac-Ir(iprpmi) $)_{3}: 2.62 \mathrm{eV}$. Le spectre d'EL de cette diode est centré à $472 \mathrm{~nm}$ conduisant à un $\mathrm{CIE}$ de $(0.18,0.38)$ signant une émission de lumière plutôt vert-bleu que réellement bleue. Dans $D 10$, trois couches additionnelles (TAPC et TCTA entre l'anode et l'EML et 1,3,5-Tri[(3-pyridyl)-phen-3-yl]benzène (TmPyPB) entre I'EML et la cathode) aident à l'injection, au transport et au blocage des porteurs de charge. En 2020, I'hôte H3 est l'hôte universel (utilisable pour des PhOLEDs RGB) le plus performant rapporté dans la littérature.

On notera qu'avec une architecture de dispositif similaire mais deux matrices hôtes différentes H7[68] et H4[68], I'EQE de la diode D14 (27.6\%) est plus faible que celui de D11 (30.1\%). Le remplacement d'une unité carboline par une unité carbazole de $\mathbf{H 4}$ à $\mathbf{H 7}$ s'accompagne d'une faible diminution de $\mathrm{E}_{\mathrm{T}}(2.77 / 2.75 \mathrm{eV}$ pour $\mathbf{H 4 / H 7 )}$ ) et d'un élargissement du gap entre HOMO et LUMO (3.46/3.51 eV pour $\mathbf{H 4 / H 7 ) [ 6 8 ] ~ p r i n c i p a l e m e n t ~ d u ~ f a i t ~ d e ~ l ' a u g m e n t a t i o n ~ d u ~ n i v e a u ~ d e ~ l a ~ L U M O ~ ( - ~}$ 2.79/-2.58 eV pour $\mathbf{H 4 / H 7 ) ~ r e n d a n t ~ l ' i n j e c t i o n ~ d e s ~ e ́ l e c t r o n s ~ p l u s ~ d i f f i c i l e s ~ d a n s ~} \mathbf{H 7}$ que dans $\mathbf{H 4}$. 


\begin{tabular}{|c|c|c|c|c|c|c|c|c|}
\hline & Architecture du dispositif & $\begin{array}{l}V_{\text {on }} \\
\text { (V) }\end{array}$ & $\begin{array}{c}\mathrm{EQE}_{\max } \\
(\%)\end{array}$ & $\begin{array}{l}C E_{\max } \\
(\mathrm{cd} / \mathrm{A})\end{array}$ & $\begin{array}{c}P E_{\max } \\
(\mathrm{Im} / \mathrm{W})\end{array}$ & $\begin{array}{c}\lambda_{\max } \\
(\mathrm{nm})\end{array}$ & $\begin{array}{c}\mathrm{CIE} \\
(\mathrm{x}, \mathrm{y})\end{array}$ & Réf. \\
\hline \multicolumn{9}{|c|}{ Dispositifs monocouches bleus } \\
\hline D8 & $\begin{array}{l}\text { ITO/PEDOT:PSS(35nm)/H1:FIrpic 8\%(100nm)/ } \\
\text { LiF(1nm)/Al(100nm) }\end{array}$ & 3.0 & 20.3 & 42.2 & 26.5 & - & $\begin{array}{l}0.15 \\
0.35\end{array}$ & [64] \\
\hline D9 & $\begin{array}{l}\text { ITO/PEDOT:PSS(40nm)/H2:FIrpic } 8 \%(100 \mathrm{~nm}) / \mathrm{LiF} \\
(1.2 \mathrm{~nm}) / \mathrm{Al}(100 \mathrm{~nm})\end{array}$ & 2.5 & 17.6 & 37.8 & 37.1 & 473,500 (sh) & $\begin{array}{c}0.15 \\
0.37\end{array}$ & [65] \\
\hline \multicolumn{9}{|c|}{ Dispositifs multicouches bleus } \\
\hline D10 & $\begin{array}{l}\text { ITO/HAT-CN(10nm)/TAPC(35nm)/TCTA(8nm)/ H3:fac- } \\
\text { Ir(iprpmi })_{3} \text { 15\%(20nm)/TmPyPB(40nm)/ Liq(2nm)/Al (120 } \\
\text { nm) }\end{array}$ & 2.9 & 27.1 & 60.5 & 63.5 & 472 & $\begin{array}{l}0.18 \\
0.38\end{array}$ & [56] \\
\hline D11 & $\begin{array}{l}\text { ITO/PEDOT:PSS(60nm)/TAPC(20nm)/mCP(10nm)/H4:FIrpic } \\
\text { 10\%(25nm)/TSPO1 }(35 \mathrm{~nm}) / \mathrm{LiF}(1 \mathrm{~nm}) / \mathrm{Al}(200 \mathrm{~nm})\end{array}$ & - & 30.1 & 53.6 & 50.6 & 473 & $\begin{array}{c}0.16 \\
0.33\end{array}$ & [68] \\
\hline D12 & $\begin{array}{l}\text { ITO/HAT-CN(5nm)/TAPC(50nm)/TCTA(5nm)/H5: Flrpic } 10 \\
\%(20 \mathrm{~nm}) / \mathrm{TmPyPB}(50 \mathrm{~nm}) / \mathrm{LiF}(1 \mathrm{~nm}) / \mathrm{Al}(100 \mathrm{~nm})\end{array}$ & 3.1 & 29.4 & 59.1 & 53 & - & $\begin{array}{c}0.16 \\
0.33\end{array}$ & [66] \\
\hline D13 & $\begin{array}{l}\text { ITO(120nm)/PEDOT:PSS(60nm)/TAPC(30nm)/ H6:Ir(dbi })_{3} \\
\text { 10\%(25nm)/TSPO1(5nm)/TPBI(30nm)/ } \\
\text { LiF(1nm)/Al(200nm) }\end{array}$ & - & 27.8 & - & 43 & 471 & - & [67] \\
\hline D14 & $\begin{array}{l}\text { ITO/PEDOT:PSS }(60 \mathrm{~nm}) / \mathrm{TAPC}(20 \mathrm{~nm}) / \mathrm{mCP} \\
(10 \mathrm{~nm}) / \mathrm{H} 7: \text { FIrpic } 10 \%(25 \mathrm{~nm}) / \mathrm{TSPO} 1(35 \mathrm{~nm}) / \mathrm{LiF} \\
(1 \mathrm{~nm}) / \mathrm{Al}(200 \mathrm{~nm})\end{array}$ & - & 27.6 & 48 & - & 473 & $\begin{array}{l}0.16 \\
0.33\end{array}$ & [68] \\
\hline D15 & $\begin{array}{l}\text { ITO/PEDOT:PSS }(60 \mathrm{~nm}) / \mathrm{TAPC}(20 \mathrm{~nm}) / \mathrm{mCP}(10 \\
\mathrm{nm}) / \mathrm{H} 8: \mathrm{Ir}(\mathrm{cb})_{3} 5 \%(25 \mathrm{~nm}) / \mathrm{TSPO} 1(5 \mathrm{~nm}) / \mathrm{TPBI}(20 \\
\mathrm{nm}) / \mathrm{LiF} / \mathrm{Al}\end{array}$ & 3.4 & 24.7 & 28.5 & 25.6 & 462 & $\begin{array}{l}0.13 \\
0.13\end{array}$ & [69] \\
\hline
\end{tabular}

Tableau 2. Exemples de performances de dispositifs PhOLEDs à émission bleue

La diode D12[66] à dopant FIrpic atteint des performances également très élevées (EQE : 29.4 \%). La matrice hôte $\mathbf{H} 5$ présente un $\mathrm{E}_{\mathrm{T}}$ de $2.98 \mathrm{eV}$, plus élevé que celui des deux matrices précédentes et un niveau HOMO plus élevé rendant l'injection des trous plus facile dans la matrice.

Finalement, D13[67] utilise un émetteur phosphorescent bleu de type phénylimidazole, $\operatorname{Ir}(\mathrm{dbi})_{3}$, de $\mathrm{E}_{\mathrm{T}}: 2.59 \mathrm{eV}$ adapté à la matrice $\mathrm{H6}\left(\mathrm{E}_{\mathrm{T}}: 2.91 \mathrm{eV}\right)$. Le spectre d'EL de D13 est centré à $471 \mathrm{~nm}$ indiquant que l'émission bleue provient uniquement du dopant $\operatorname{lr}(\mathrm{dbi})_{3}$.

La diode D15[69] utilise un dopant phosphorescent à ligand carbénique $: \operatorname{Ir}(\mathrm{cb})_{3}$ et la matrice hôte H8. $\operatorname{Ir}(\mathrm{cb})_{3}$ possède un $E_{\mathrm{T}}$ de $2.72 \mathrm{eV}$ plus élevé que ceux des dopants bleus précédemment décrits. La matrice $\mathbf{H 8}$ possède un $E_{\mathrm{T}}$ de $3.02 \mathrm{eV}$ adapté à ce nouveau dopant. Avec un $\mathrm{V}_{\text {on }}$ de $3.4 \mathrm{~V}, \mathrm{D} 15$ atteint un $E Q E_{\max }$ de $24.7 \%$ et émet un bleu profond de coordonnées chromatiques $(0.13,0.13)$. D15 est la diode la plus efficace émettrice d'un bleu profond.

Ces quelques exemples montrent l'efficacité des transferts d'énergie des matrices hôtes vers les dopants phosphorescents émetteurs de lumière bleue.

\subsection{Emission verte des OLEDs phosphorescentes}

Quelques PhOLEDs monocouches émettrices de lumière verte ont été rapportées dans la littérature, elles atteignent des EQE supérieurs à $13 \%$ (Tableau 3) mais sont moins efficaces que les diodes monocouches bleues rapporté ci-dessus (D8-D9, EQE >17,6\%). Ceci est attribué à des recherches plus poussées dans le domaine des OLEDs émettrices de lumière bleue. On notera que l' $\mathrm{E}_{\mathrm{T}}$ des deux dopants phosphorescents verts utilisés dans les dispositifs D16-D23 [56, 65, $70,71,72,73]$ est de l'ordre de $2.4 \mathrm{eV}$ soit $0.2 \mathrm{eV}$ moins élevé que l' $\mathrm{E}_{\mathrm{T}}$ des dopants bleus précédents (Figure 6). La contrainte sur l'adéquation des niveaux $\mathrm{E}_{\mathrm{T}}$ matrice hôte/dopant est donc moins forte.

Avec la même structure de diode, PEDOT:PSS comme HIL et LiF comme EIL, les performances de D16-

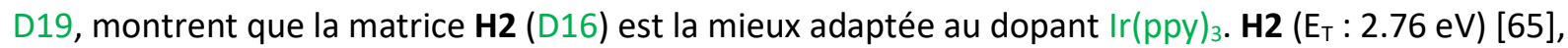
$\mathbf{H 9}\left(\mathrm{E}_{\mathrm{T}}: 2.74 \mathrm{eV}\right)[70]$ et $\mathbf{H 1 0}\left(\mathrm{E}_{\mathrm{T}}: 2.78 \mathrm{eV}\right)$ [71] ont un $\mathrm{E}_{\mathrm{T}}$ supérieur à celui de $\mathbf{H 1 1}\left(\mathrm{E}_{\mathrm{T}}: 2.64 \mathrm{eV}\right)$ [71] assurant un bon transfert d'énergie $\mathrm{T}_{1}^{\mathrm{H}} \rightarrow \mathrm{T}^{\mathrm{G}}{ }_{1}$. La meilleure performance de $\mathrm{D} 16$ par rapport à $\mathrm{D} 18$ en terme d'EQE et de $\mathrm{V}_{\text {on }}$ provient de la différence des niveaux HOMO/LUMO de ces deux SCOs, en effet, l'injection des charges est plus facile à la fois à l'anode et à la cathode dans H2 (HOMO/LUMO de 5.40/-2.46 eV) que dans H10 (HOMO/LUMO : -6.0/-2.10 eV). En comparant $\mathbf{H 1 0}$ et $\mathbf{H 1 1}$, la présence des deux unités carbazole sur le fluorène de $\mathbf{H 1 1}$ remonte le niveau de sa HOMO sans variation de sa LUMO (HOMO/LUMO de H11: -5.51/-2.10 eV) qui est principalement localisée sur l'unité fluorène/oxyde de phosphine. La position $\mathrm{C} 4$ du fluorène est une position très intéressante car elle permet 
de rompre partiellement la conjugaison entre le fluorène et son substituant ce qui permet d'assurer un contrôle fin des niveaux d'énergie des orbitales frontières. $[59,74-76]$ Les performances de D18 et D19 sont identiques (EQE : 13.3/13.2 \%) avec toutefois un $\mathrm{V}_{\text {on }}$ plus faible (2.4 V vs 3.3 V) pour D19 que pour D18 signant l'injection plus facile des charges dans H11 que dans H10.

$\mathbf{H} 2$ possède, comparé à $\mathbf{H}$, un $\mathrm{E}_{\mathrm{T}}$ similaire (2.74 vs $2.76 \mathrm{eV}$ ) mais une $\mathrm{HOMO}$ plus profonde (-5.71 vs $-5.40 \mathrm{eV}$ ) rendant l'injection des trous plus difficiles dans D17[70] que dans D16[65]. L'injection plus difficile entraine une augmentation du $V_{\text {on }}(3.4$ vs $2.3 \mathrm{eV}$ ) et une baisse d'EQE (14.6 vs $15.6 \%)$. Ces quelques exemples de diodes monocouches phosphorescentes vertes mettent en évidence l'importance des niveaux d'HOMO, LUMO et $\mathrm{E}_{\mathrm{T}}$ des matrices hôtes sur les performances des dispositifs.

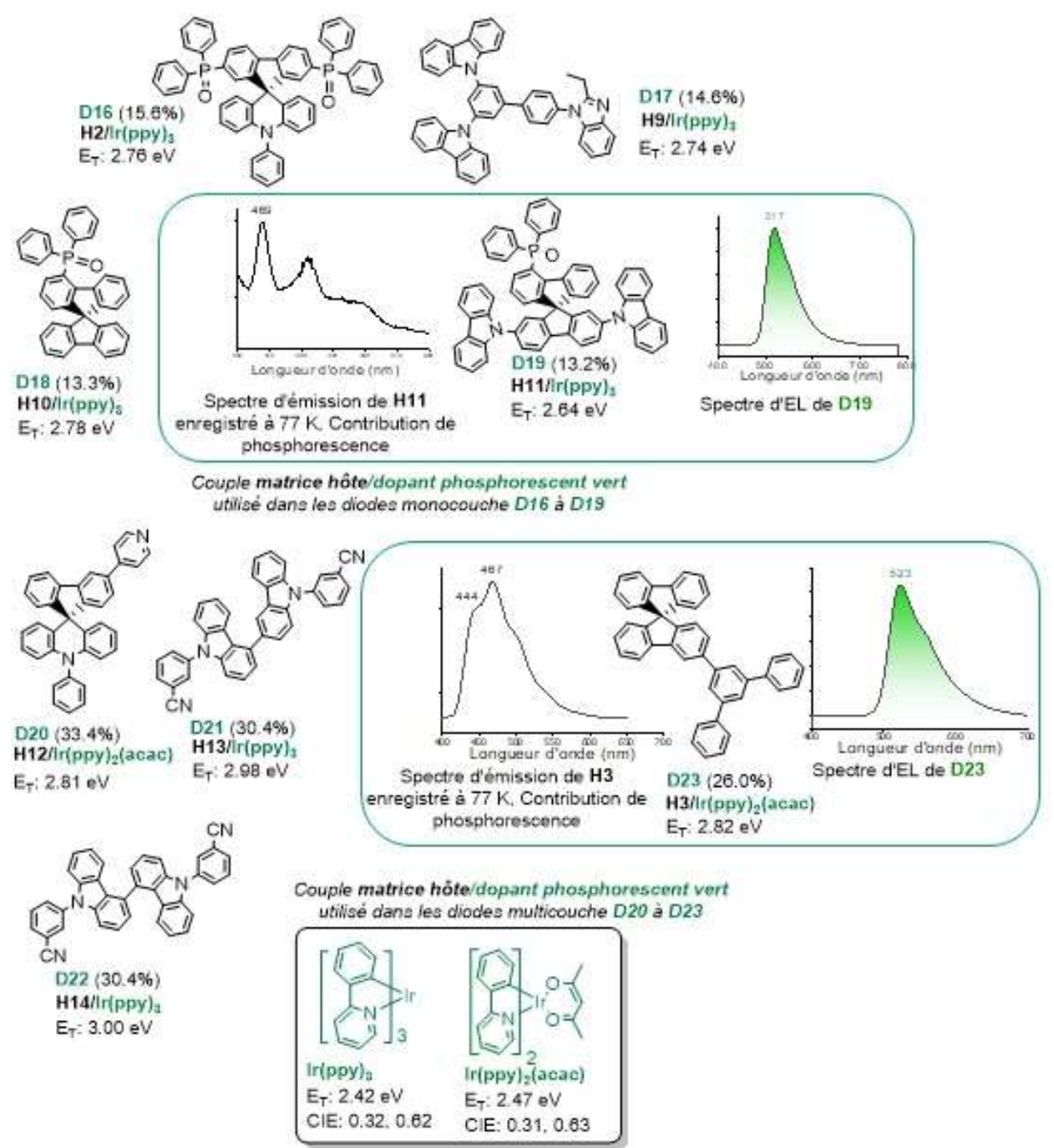

Figure 6. Exemples de couple matrice hôte/dopant phosphorescent vert

D20-D23 sont des diodes multicouches très performantes utilisant comme matrice hôte H12 [72] ou deux matrices hôtes isomères de position ( $\mathbf{H 1 3}$ et $\mathbf{H 1 4}$ ) [73] ou à nouveau la matrice $\mathrm{PHC} \mathbf{H 3}$ [56] (utilisée précédemment dans le dispositif bleu D10). Alors que l' $E_{\mathrm{T}}$ de $\mathbf{H 1 2}$ (2.81 eV) est plus bas que celui des deux isomères (2.98/3.00 eV pour H13/H14), c'est D20, utilisant $\mathbf{H 1 2}$ comme matrice hôte qui présente les meilleures performances en terme de $V_{\text {on }}$ et d'EQE. Cela montre qu'un haut $E_{T}$ de la matrice est loin d'être suffisant pour atteindre une haute performance. Ceci peut être expliqué (i) par une meilleure injection des trous dans H12 (HOMO : $-5.69 \mathrm{eV}$ ) que dans les deux isomère dérivés du carbazole (HOMO: -5.90/-6.11 eV pour H13/H14), (ii) par un meilleur confinement des charges dans l'EML dû aux différentes couches intermédiaires dans D20 que dans D21-D22 et enfin (iii) par les propriétés intrinsèques (HOMO/LUMO, rendement quantique d'émission, temps de vie,...) du dopant vert : Ir(ppy $)_{3}$ pour D21-22 et Ir(ppy $)_{2}($ acac) pour D20. Ces travaux montrent les réglages fins qu'il faut effectuer pour pouvoir atteindre de hautes performances. 


\begin{tabular}{|c|c|c|c|c|c|c|c|}
\hline & Architecture du dispositif & $\begin{array}{l}V_{\text {on }} \\
\text { (V) }\end{array}$ & $\begin{array}{c}E_{\text {EQ }} \\
(\%)\end{array}$ & $\begin{array}{l}C E_{\max } \\
(\mathrm{cd} / \mathrm{A}) \\
\end{array}$ & $\begin{array}{l}P E_{\max } \\
(\mathrm{Im} / \mathrm{W})\end{array}$ & $\begin{array}{c}\mathrm{CIE} \\
(\mathrm{x}, \mathrm{y})\end{array}$ & Réf. \\
\hline \multicolumn{8}{|c|}{ Dispositifs monocouches vert } \\
\hline D16 & $\begin{array}{l}\text { ITO/PEDOT:PSS(40nm)/H2:Ir(ppy) }{ }_{3} \text { 10\%(100nm)/LiF }(1.2 \mathrm{~nm}) / \mathrm{Al} \\
(100 \mathrm{~nm})\end{array}$ & 2.3 & 15.6 & 52.9 & 52 & $\begin{array}{l}0.31 \\
0.63\end{array}$ & [65] \\
\hline D17 & ITO/PEDOT:PSS(40nm)/H9:Ir(ppy) ${ }_{3}$ 10\%(90nm)/LiF(0.5nm)/ Mg : Ag & 3.4 & 14.6 & 52.3 & 35.4 & $\begin{array}{l}0.30 \\
0.64\end{array}$ & [70] \\
\hline D18 & $\begin{array}{l}\text { ITO/PEDOT:PSS(40nm)/H10:Ir(ppy) }{ }_{3} \text { 10\%(100nm)/LiF(1.2nm)/Al } \\
(100 \mathrm{~nm})\end{array}$ & 3.3 & 13.3 & 50.2 & 29.8 & - & [71] \\
\hline D19 & $\begin{array}{l}\text { ITO/PEDOT:PSS(40nm)/H11:Ir(ppy) })_{3} \text { 10\%(100nm)/LiF(1.2nm)/Al } \\
(100 \mathrm{~nm})\end{array}$ & 2.4 & 13.2 & 45.8 & 49.6 & $\begin{array}{c}0.30 \\
0.64\end{array}$ & [71] \\
\hline \multicolumn{8}{|c|}{ Dispositif multicouche vert } \\
\hline D20 & $\begin{array}{l}\text { ITO/HAT-CN(10nm)/TAPC(40nm)/TCTA(10nm)/H12: Ir (ppy)2(acac) } \\
(20 \mathrm{~nm}) / \operatorname{TmPyPB}(50 \mathrm{~nm}) / \mathrm{Liq}(2 \mathrm{~nm}) / \mathrm{Al}(120 \mathrm{~nm})\end{array}$ & 2.1 & 33.4 & 118.8 & 148.8 & - & [72] \\
\hline D21 & $\begin{array}{l}\text { ITO }(120 \mathrm{~nm}) / \mathrm{PEDOT}: \mathrm{PSS}(60 \mathrm{~nm}) / \mathrm{TAPC}(20 \mathrm{~nm}) / \mathrm{mCP}(10 \mathrm{~nm}) / \mathrm{H} 13: \operatorname{Ir}(\mathrm{PPy})_{3} \\
5 \%(25 \mathrm{~nm}) / \mathrm{TSPO} 1(35 \mathrm{~nm}) / \mathrm{LiF}(1 \mathrm{~nm}) / \mathrm{Al}(200 \mathrm{~nm})\end{array}$ & 4.0 & 30.4 & 93.6 & 50 & - & [73] \\
\hline $\mathrm{D} 22$ & $\begin{array}{l}\text { ITO(120nm)/PEDOT:PSS }(60 \mathrm{~nm}) / \mathrm{TAPC}(20 \mathrm{~nm}) / \mathrm{mCP}(10 \mathrm{~nm}) / \mathrm{H} 14: \operatorname{Ir}(\mathrm{PPy})_{3} \\
5 \%(25 \mathrm{~nm}) / \mathrm{TSPO} 1(35 \mathrm{~nm}) / \mathrm{LiF}(1 \mathrm{~nm}) / \mathrm{Al}(200 \mathrm{~nm})\end{array}$ & 4.0 & 30.4 & 93.2 & 46.2 & - & [73] \\
\hline D23 & $\begin{array}{l}\text { ITO/HAT-CN(10nm)/TAPC(35nm)/TCTA(8nm)/ H3:Ir(ppy) } 2 \text { (acac) } \\
\text { 15\%(20nm)/TmPyPB }(40 \mathrm{~nm}) / \mathrm{Liq}(2 \mathrm{~nm}) / \mathrm{Al}(120 \mathrm{~nm})\end{array}$ & 2.9 & 26.0 & 96.0 & 101.2 & $\begin{array}{l}0.32 \\
0.63\end{array}$ & [56] \\
\hline
\end{tabular}

Tableau 3. Exemples de performances de dispositifs PhOLEDs à émission verte

On notera enfin que les deux isomères de position $\mathbf{H 1 3}$ et $\mathbf{H 1 4}$, du fait de la rupture de conjugaison assurée par la substitution du carbazole en $\mathrm{C} 4$, possèdent des propriétés électroniques similaires. De ce fait, les diodes D21 et D22 ont des performances comparables.

Comparée à la diode D20 utilisant comme EML la matrice ambipolaire d'architecture de type Donneur-Spiro-Accepteur (D-spiro-A), H12:Ir(ppy) 2 (acac), la diode D23 utilisant le couple

H3:Ir(ppy $)_{2}(\mathrm{acac})$ présente des performances très élevées pour une matrice PHC. Le design D-spiro-A a beaucoup été développé par notre équipe car il s'avère très performant pour contrôler les propriétés électroniques et tout particulièrement les niveaux HOMO/LUMO des SCOs.[77-80] Malgré un écart HOMO/LUMO plus élevé dans la matrice H3 (3.95 eV) que dans la matrice $\mathbf{H 1 2}$ (3.51 eV), le $V_{\text {on }}$ du dispositif D23 [56] reste faible (2.9 V) et son EQE atteint $26 \%$ montrant la potentialité d'utiliser des matrices hôtes PHC dans les PhOLEDs.

Ces quelques exemples montrent que les diodes phosphorescentes multicouches vertes utilisant les couches additionnelles à base de TAPC, TCTA, MCP, TmPyPB ou TSPO1, présentent des performances comparables à celles des meilleures diodes phosphorescentes multicouches bleues (D10-D11) avec des EQE pouvant dépasser $30 \%$.

\subsection{Emission jaune/orange des OLEDs phosphorescentes}

Des diodes émettrices de lumière jaune/orange sont aussi décrites dans la littérature avec des performances approchant $8 \%$ en diode monocouche et dépassant $26 \%$ en diode multicouche (Tableau 4). Les dopants phosphorescents jaune/orange ont des $\mathrm{E}_{\mathrm{T}}$ de l'ordre de $2.2 \mathrm{eV}$ (Figure 7). Avec un $E_{T}$ de 2,3 eV, $\mathbf{H 1 5}$ paraît être une matrice hôte adaptée au dopant bis(2-(9,9-diéthyl-fluorèn2-yl)-1-phényl-1H-benzo[d ]imidazolato)(acétylacétonate)iridium(III) ((fbi) $2 \mathrm{Ir}(\mathrm{acac}), \mathrm{E}_{\mathrm{T}}: 2.18 \mathrm{eV}$ ). La PhOLED D24 émet dès $3.1 \mathrm{eV}$ et atteint un EQE de $7.8 \%$.[81] Ces performances restent malgré tout modestes.

D25 a comme matrice hôte $\mathbf{H 1 6}$ qui possède un atome de carbone $\mathrm{sp}^{3}$ pour relier l'unité benzimidazoindole (BII) accepteur d'électrons aux deux unités triphenylamine (TPA) donneuses d'électrons.[66] Cette architecture moléculaire de type " $D-\sigma-A$ » confère à $H 16$ un $E_{T}$ de $2.95 \mathrm{eV}$ adapté au dopant iridium(III) bis(4-phenylthieno[3,2-c]pyridinato- $\left.N, C 2^{\prime}\right)$ acétylacétonate) (PO-01, $\mathrm{E}_{\mathrm{T}}: 2.2 \mathrm{eV}$ ). D25 émet de la lumière orange dès $2.6 \mathrm{~V}$ et atteint un $\mathrm{EQE}_{\max }$ proche de $30 \%$. Il est intéressant de noter que $\mathbf{H 1 6} \mathrm{a}$ également été utilisé comme matrice hôte pour des émetteurs phosphorescents bleus (dopant FIrpic), verts $\left(\operatorname{Ir}(\mathrm{ppy})_{2}(\mathrm{acac})\right)$ et rouges $\left(\operatorname{Ir}(\mathrm{piq})_{2}(\mathrm{acac})\right)$ avec des EQE atteignant respectivement 21.2, 26.4 et $22.1 \%$ [66]. La matrice $\mathbf{H 1 6}$ peut donc être considérée comme une " matrice hôte universelle ", c'est-à-dire adaptée à toutes les couleurs. On notera que $\mathrm{H} 16$ a une structure proche de celle de H5 (Figure 5), avec deux unités triphénylamines au lieu de deux unités carbazole. La matrice $\mathbf{H 5}$ a été utilisée avec le dopant FIrpic (D12), mais aussi avec des dopants verts $\left(\operatorname{Ir}(\mathrm{ppy})_{2}(\mathrm{acac})\right)$, jaune/orange 
(PO-01/Ir(2-phq $)_{3}$ ) ou rouges (Ir(piq) $\left.)_{2}(\mathrm{acac})\right)[66]$ dans des dispositifs efficaces, $\mathbf{H} \mathbf{5}$ est donc aussi une " matrice hôte universelle ». Il est important de mentionner que si théoriquement une matrice à $\mathrm{E}_{\mathrm{T}}>$ $2.75 \mathrm{eV}$ peut être utilisée pour tous les dopants RGB, en pratique si le $E_{T}$ est trop haut, les dopants rouges peuvent s'avérer peu efficaces. D'autre part, l'ajustement des HOMO/LUMO est aussi capital afin d'obtenir de très hautes performances rendant cette problématique beaucoup plus compliquée qu'elle n'y parait.

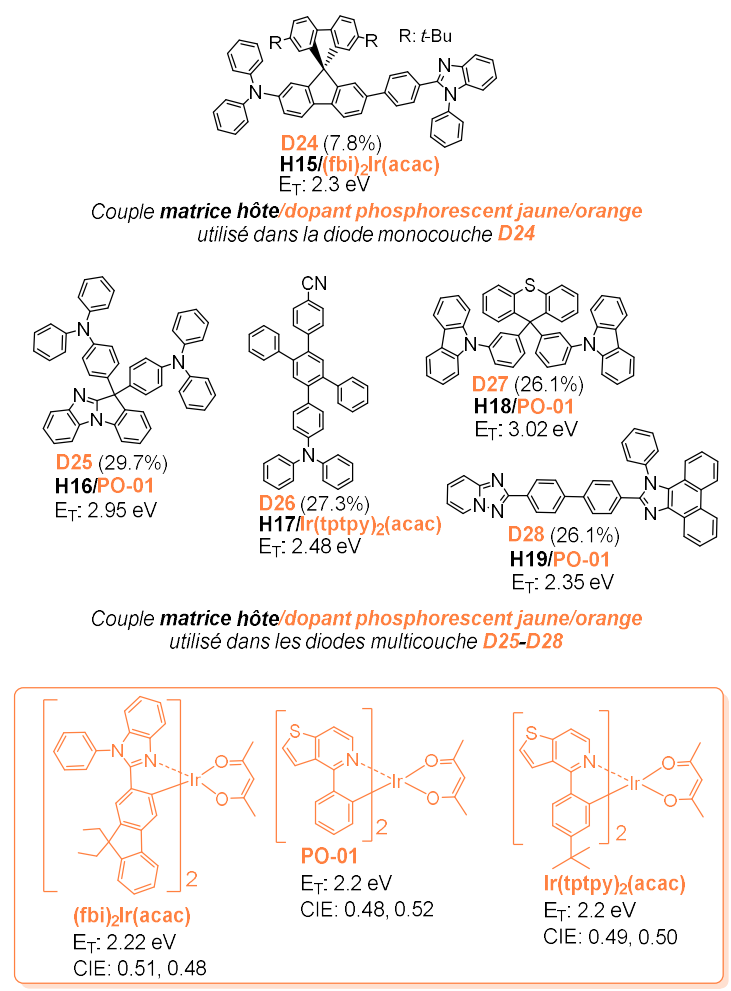

Figure 7. Exemples de couple matrice hôte/dopant phosphorescent jaune/orange

H17 est un SCO bipolaire dont les deux unités $D$ et $A$ sont séparées par un lien oligophénylène.[82] Ce SCO est un émetteur fluorescent bleu profond $(0.15,0.08)$ lorsqu'il est utilisé seul dans l'EML.[82] Son $E_{\mathrm{T}}$ de $2.48 \mathrm{eV}$ est adapté à l'émetteur phosphorescent orange acétylacétonato-bis(4-(4-tertbutylphényl)-thiéno[3,2-c]pyridinato-C2,N)iridium (Ir(tptpy) 2 (acac), $\mathrm{E}_{\mathrm{T}}: 2.20 \mathrm{eV}$ ) et permet des transfert d'énergie vers ce dernier. Les performances de $D 26\left(V_{\text {on }}: 2.6 \mathrm{~V}\right.$ et EQE $\left.E_{\max }: 27.3 \%\right)$ sont excellentes. Les auteurs rapportent pour cette diode une luminance maximum dépassant 110000 $\mathrm{cd} / \mathrm{m}^{2}$ (environ 10 fois plus élevée que celle des diodes précédemment décrites) et une grande stabilité de l'émission à $1000 \mathrm{~cd} / \mathrm{m}^{2}$ qui font de $D 26$ une diode phosphorescente orange particulièrement intéressante. Dans cette diode, le 1,3-bis[3,5-di(pyridin-3-yl)-phényl]benzène (BmPyPB) est utilisé comme ETL.

\begin{tabular}{|c|c|c|c|c|c|c|c|c|}
\hline & Architecture du dispositif & $\begin{array}{l}V_{\text {on }} \\
\text { (V) }\end{array}$ & $\begin{array}{c}E_{\text {EQ }} \\
(\%)\end{array}$ & $\begin{array}{l}\mathrm{CE}_{\max } \\
(\mathrm{cd} / \mathrm{A}) \\
\end{array}$ & $\begin{array}{l}P E_{\max } \\
(\mathrm{Im} / \mathrm{W})\end{array}$ & $\begin{array}{l}\lambda_{\max } \\
(\mathrm{nm})\end{array}$ & $\begin{array}{c}\text { CIE } \\
(x, y)\end{array}$ & Réf. \\
\hline \multicolumn{9}{|c|}{ Dispositif monocouche jaune/orange } \\
\hline D24 & ITO/ H15 : (fbi) 2 lr(acac) $5 \% / L i F(1 n m) / A l(150 n m)$ & 3.1 & 7.8 & 22 & 7.5 & 572 & $0.53,0.47$ & [81] \\
\hline \multicolumn{9}{|c|}{ Dispositif multicouche jaune/orange } \\
\hline D25 & $\begin{array}{l}\text { ITO/HAT-CN }(5 \mathrm{~cm}) / \text { TAPC }(50 \mathrm{~nm}) / \text { TCTA }(5 \mathrm{~nm}) / \text { H16: } \\
\text { PO-01 } 10 \%(20 \mathrm{~nm}) / \mathrm{TmPyPB}(50 \mathrm{~nm}) / \mathrm{LiF}(1 \mathrm{~nm}) / \mathrm{Al} \\
(100 \mathrm{~nm})\end{array}$ & 2.6 & 29.7 & 80.3 & 79.9 & $\begin{array}{l}572 \\
600(s h)\end{array}$ & $0.52,0.48$ & [66] \\
\hline D26 & $\begin{array}{l}\text { ITO/HAT-CN }(5 \mathrm{~nm}) / \mathrm{TAPC}(50 \mathrm{~nm}) / \mathrm{TCTA}(5 \mathrm{~nm}) / \mathrm{H} 17: \\
\operatorname{lr}(\mathrm{tptpy})_{2}(\mathrm{acac}) 3 \%(20 \mathrm{~nm}) / \mathrm{BmPyPB}(40 \mathrm{~nm}) / \mathrm{LiF} \\
(1 \mathrm{~nm}) / \mathrm{Al}\end{array}$ & 2.6 & 27.3 & 86.7 & 104.7 & 560 & $0.49,0.51$ & [82] \\
\hline D27 & $\begin{array}{l}\text { ITO/TAPC(40nm)/ H18 : PO-01 6\% (10nm) } \\
/ \text { TmPyPB(40nm) / LiF (1nm) /Al(100nm) }\end{array}$ & 3.0 & 26.1 & 80.9 & 79.4 & & $0.49,0.51$ & [83] \\
\hline D28 & $\begin{array}{l}\text { ITO/TAPC }(30 \mathrm{~nm}) / \mathrm{TCTA}(5 \mathrm{~nm}) / \mathrm{mCP}(5 \mathrm{~nm}) / \mathrm{H} 19: \\
\text { PO-01 3\% }(20 \mathrm{~nm}) / \mathrm{TPBI}(50 \mathrm{~nm}) / \mathrm{LiF}(1 \mathrm{~nm}) / \mathrm{Al} \\
(100 \mathrm{~nm})\end{array}$ & 2.6 & 26.1 & 80.6 & 82.4 & 568 & $0.51,0.48$ & [84] \\
\hline
\end{tabular}

Tableau 4. Exemples de performances de dispositifs PhOLEDs à émission jaune/orange

Bien que $\mathbf{H 1 8}$ [83] possède un $E_{T}$ beaucoup plus élevé que celui de $\mathbf{H 1 9}$ [84], 3.02 vs 2.35 eV, D27 et 
D28 présentent des performances similaires ( $\left.E Q E_{\max }: 26.1 \%\right)$ mettant en évidence comme précédemment que ce paramètre n'est ici plus prépondérant. Les niveaux HOMO/LUMO des deux matrices (-5.64 eV/-2.13 eV pour H18 et $-5.37 \mathrm{eV} /-2.36 \mathrm{eV}$ pour $\mathbf{H 1 9}$ ) rendent compte d'une injection plus facile des charges dans $\mathbf{H 1 9}$ que dans $\mathbf{H 1 8}$. Les deux dispositifs utilisent le TAPC comme HTL et EBL et le TmPyPB (D27) ou le TPBI (D28) comme ETL. D28 utilise en plus une couche TCTA comme HTL et une couche de mCP comme EBL. Ces deux couches supplémentaires facilitent l'injection et le transport des charges et leur recombinaison dans la couche active entrainant une diminution du $\mathrm{V}_{\text {on }}$ de $0.4 \mathrm{eV}$ (3.0 V pour D27 et 2.6 V pour D28).

\subsection{Emission rouge des OLEDs phosphorescentes}

Les trois dopants phosphorescents rouges utilisés dans les diodes D29-D35 [56, 70, 82, 85, 86] ont un $E_{T}$ de l'ordre de $2.0 \mathrm{eV}$ et peuvent donc doper des matrices possédant des $E_{T}$ encore moins élevés que les matrices utilisées pour le bleu, le vert et le jaune/orange (Figure 8). Avec une structure de dispositif monocouche similaire et un même dopant phosphorescent rouge bis(2-

méthyldibenzo[f,h]quinoxaline)(acétylacétonate) iridium(III), Ir(MDQ) 2 (acac), D29 et D30 [70] montrent des performances similaires avec un EQE proche de 15\% (Tableau 5). Malgré un niveau $E_{T}$ plus élevé et un écart HOMO/LUMO moins large pour H21 que pour H20, les performances des PhOLEDs sont les mêmes mettant en exergue la difficulté de concevoir des matériaux hôtes pour PhOLEDs.

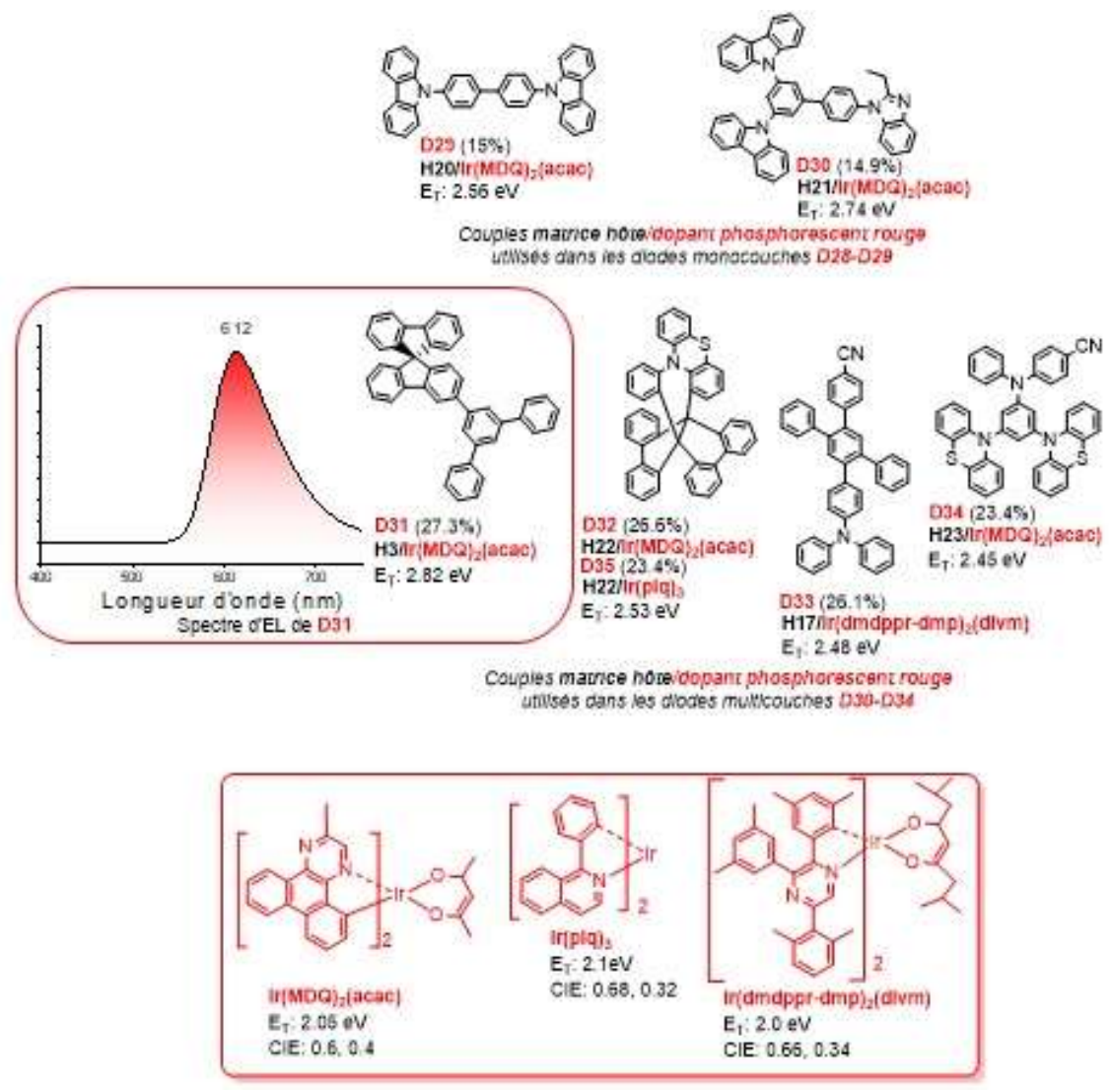

Figure 8. Exemples de couple matrice hôte/dopant phosphorescent rouge

Les diodes multicouches D31[56], D32[85] et D34[86] à base du même dopant rouge $\operatorname{Ir}(M D Q)_{2}$ (acac), présentent des performances assez similaires variant plus du fait de la différence d'architecture des dispositifs que par les propriétés électroniques des matrices $(\mathbf{H 3}, \mathbf{H 2 2}$ et $\mathbf{H 2 3}$ ) qui possèdent toutes les trois un $\mathrm{E}_{\mathrm{T}}$ supérieur à $2.5 \mathrm{eV}$.

D33[82] utilise un autre dopant rouge, Ir(dmdppr-dmp $)_{2}\left(\right.$ divm) et le $E_{T}$ de $\mathbf{H 1 7}$, matrice déjà utilisée pour un dopant jaune/orange dans D26, est du même ordre de grandeur que celui des autres matrices $(2.48 \mathrm{eV})$. La performance de D33 similaire à celle des autres présente le $V_{\text {on }}$ le plus faible de la série (2.6 V). Ce $V_{\text {on }}$ est similaire à celui de la diode jaune-orange D26 mais la luminance maximale 
atteinte par D33 n'est que de $40532 \mathrm{~cd} / \mathrm{m}^{2}$ (deux fois plus faible que celle atteinte par D26).

\begin{tabular}{|c|c|c|c|c|c|c|c|c|}
\hline & Architecture du dispositif & $\begin{array}{l}V_{\text {on }} \\
\text { (V) }\end{array}$ & $\begin{array}{c}\mathrm{EQE}_{\max } \\
(\%)\end{array}$ & $\begin{array}{l}C E_{\max } \\
\text { (cd/A) }\end{array}$ & $\begin{array}{c}P_{\max } \\
(\mathrm{Im} / \mathrm{W})\end{array}$ & $\begin{array}{l}\lambda_{\max } \\
(\mathrm{nm})\end{array}$ & $\begin{array}{c}\mathrm{CIE} \\
(\mathrm{x}, \mathrm{y})\end{array}$ & Réf. \\
\hline \multicolumn{9}{|c|}{ Dispositif monocouche Rouge } \\
\hline D29 & $\begin{array}{l}\text { ITO/PEDOT:PSS(40nm)/H20:Ir(MDQ) }{ }_{2} \text { acac } 5 \%(90 \mathrm{~nm}) / \\
\mathrm{LiF}(0.5 \mathrm{~nm}) / \mathrm{Mg}: \mathrm{Ag}\end{array}$ & 3.0 & 15 & 23.7 & 21.3 & & $0.61,0.39$ & [70] \\
\hline D30 & $\begin{array}{l}\text { ITO/PEDOT:PSS(40nm)/H21:Ir(MDQ) } 2 \text { acac } 5 \%(90 \mathrm{~nm}) / \\
\mathrm{LiF}(0.5 \mathrm{~nm}) / \mathrm{Mg}: \mathrm{Ag}\end{array}$ & 3.2 & 14.9 & 23.5 & 21.1 & & $0.61,0.39$ & [70] \\
\hline \multicolumn{9}{|c|}{ Dispositif multicouche Rouge } \\
\hline D31 & $\begin{array}{l}\text { ITO/HAT-CN }(10 \mathrm{~nm}) / \mathrm{TAPC}(45 \mathrm{~nm}) / \mathrm{TCTA}(8 \mathrm{~nm}) / \mathrm{H3}: \\
\operatorname{Ir}(\mathrm{MDQ})_{2 \mathrm{acac}} 12 \%(20 \mathrm{~nm}) / \mathrm{Tm} \mathrm{PyPB}(55 \mathrm{~nm}) / \mathrm{Liq}(2 \mathrm{~nm}) / \\
\mathrm{Al}(120 \mathrm{~nm})\end{array}$ & 3.0 & 27.3 & 40 & 40 & 612 & $0.63,0.37$ & [56] \\
\hline D32 & $\begin{array}{l}\text { ITO/HAT-CN }(10 \mathrm{~nm}) / \mathrm{TAPC}(45 \mathrm{~nm}) / \mathrm{TCTA}(10 \mathrm{~nm}) / \mathrm{H} 22: \\
\operatorname{Ir}(\mathrm{MDQ})_{2}(\mathrm{acac}) 6 \%(20 \mathrm{~nm}) / \mathrm{TmPyPB}(45 \mathrm{~nm}) / \mathrm{Liq}(2 \mathrm{~nm}) / \\
\mathrm{Al}(120 \mathrm{~nm})\end{array}$ & 4.0 & 26.6 & 47.3 & 40.2 & & $0.62,0.38$ & [85] \\
\hline D33 & 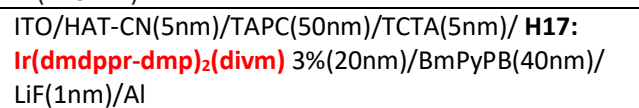 & 2.6 & 26.1 & 30.5 & 36.8 & 614 & $0.66,0.34$ & [82] \\
\hline D34 & $\begin{array}{l}\text { ITO/HAT-CN }(10 \mathrm{~nm}) / \mathrm{TAPC}(50 \mathrm{~nm}) / \mathrm{H} 23: \operatorname{Ir}(\mathrm{MDQ})_{2} \text { (acac) } \\
2.5 \%(20 \mathrm{~nm}) / \mathrm{TPBI}(55 \mathrm{~nm}) / \mathrm{Liq}(2 \mathrm{~nm}) / \mathrm{Al}(120 \mathrm{~nm})\end{array}$ & 3.04 & 23.4 & 42.5 & 44.3 & & $0.60,0.40$ & [86] \\
\hline D35 & $\begin{array}{l}\text { ITO/HAT-CN }(10 \mathrm{~nm}) / \mathrm{TAPC}(45 \mathrm{~nm}) / \mathrm{TCTA}(10 \mathrm{~nm}) / \mathrm{H22}: \\
\operatorname{Ir}(\text { piq) })_{3} 6 \%(20 \mathrm{~nm}) / \mathrm{TmPyPB}(45 \mathrm{~nm}) / \mathrm{Liq}(2 \mathrm{~nm}) / \mathrm{Al} \\
(120 \mathrm{~nm})\end{array}$ & 4.7 & 23.4 & 20 & 15.8 & & $0.67,0.33$ & [85] \\
\hline
\end{tabular}

Tableau 5. Exemples de performances de dispositifs PhOLEDs à émission rouge

D32 [85] et D35[85], ont une structure de dispositif similaire et une matrice hôte identique, H22, mais un dopant rouge différent : $\operatorname{Ir}(\mathrm{MDQ})_{2}(\mathrm{acac})$ dans $\mathrm{D} 32$ et $\operatorname{Ir}(\mathbf{p i q})_{3}$ dans $\mathrm{D} 35$. Les deux diodes ne présentent pas les mêmes performances : on observe une augmentation de $V_{\text {on }}$ et diminution d'EQE, lorsque l'on passe de D32 à D35. Ces deux exemples montrent que la nature du dopant joue aussi un rôle prépondérant sur les performances du fait (i) de ses propriétés intrinsèques (HOMO/LUMO, rendement quantique d'émission, temps de vie,...), (ii) d'interactions matrice hôte/dopant différentes et (iii) de mobilité voire d'injection de charge différents en fonction des couples matrice hôte/dopant.

\subsection{Emission blanche des OLEDS phosphorescentes}

Les dispositifs PhOLEDs émetteurs de lumière blanche peuvent être classés en deux familles en fonction de l'origine de l'émission. La première dans laquelle deux dopants phosphorescents de couleur complémentaire (souvent bleu et orange) sont incorporés dans une même matrice hôte, on parlera alors de PhOLEDs blanches à dopant mixte (W-D36[66], W-D37[72], W-D39[87], W-D40[83]) (Figure 9, Tableau 6). Dans ces diodes, l'émission provient des deux dopants phosphorescents. La seconde dans laquelle la matrice hôte dopée par un émetteur phosphorescent est elle-même fluorescente, on parlera d'OLEDs phosphorescente hybride (W-D38[84]), c'est-à-dire dont l'émission provient à la fois de la matrice fluorescente et du dopant phosphorescent.

Les matrices des diodes de la première catégorie, sont en général des " matrices universelles" adaptées à la fois à un dopant phosphorescent bleu de haut $E_{T}$ et à un dopant jaune de $E_{T}$ plus faible. Dans WD-D36 [66], H5 est dopé à $10 \%$ en masse par FIrpic et à $0.6 \%$ en masse par PO-01, les deux dopants étant dispersés uniformément dans la matrice. Dans un dispositif multicouche similaire à celui de la diode émettrice de lumière bleue $D 12$, excepté la présence supplémentaire de $0.6 \%$ de dopant jaune/orange PO-01 dans H5, la diode W-D36 émet dès $3.1 \mathrm{~V}$ avec un EQE $E_{\max }$ de $29 \%$ une lumière blanche de coordonnées chromatiques $(0.39,0.46)$. Comparées aux performances de $\mathrm{D} 12$, la présence du dopant $\mathrm{PO}-01$ en faible quantité n'affecte pas l'injection et le transport des charges dans la matrice et ne modifie pas les propriétés de la diode mais uniquement la couleur de la lumière émise. 


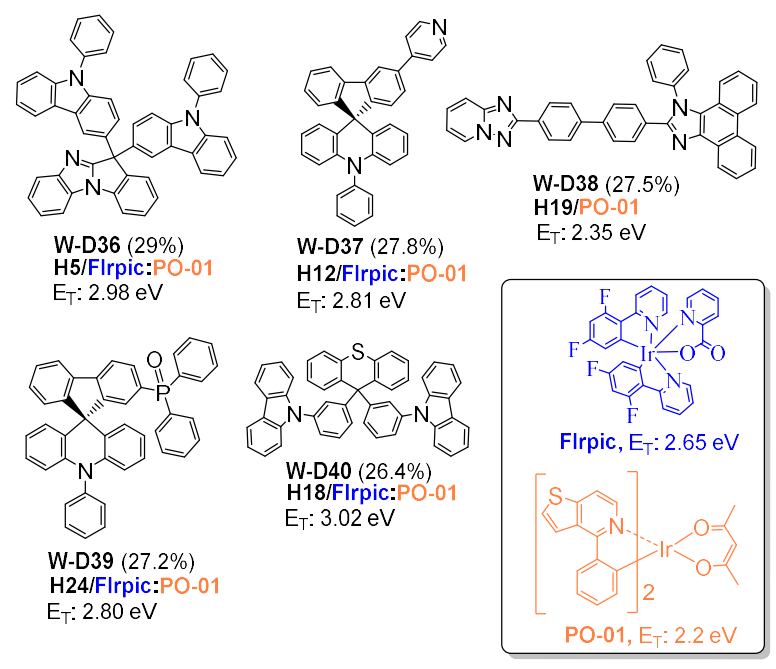

Couple matrice hote/dopants pour diode blanche multicouche

Figure 9. Exemples de couple matrice hôte/dopant phosphorescent pour des PhOLEDs blanches

\begin{tabular}{|c|c|c|c|c|c|c|c|}
\hline & Architecture du dispositif & $\begin{array}{l}V_{\text {on }} \\
(V)\end{array}$ & $\begin{array}{l}E E_{\max } \\
(\%)\end{array}$ & $\begin{array}{l}C E_{\max } \\
(\mathrm{cd} / \mathrm{A})\end{array}$ & $\begin{array}{l}P E_{\max } \\
(\operatorname{Im} / \mathrm{W})\end{array}$ & $\begin{array}{l}\mathrm{CIE} \\
(\mathrm{x}, \mathrm{y})\end{array}$ & Réf. \\
\hline W-D36 & $\begin{array}{l}\text { ITO/HAT-CN(5 cm)/TAPC(50 nm)/TCTA(5 nm)/H5 } \\
: \text { FIrpic:PO-01(10\%,0.6\%,20nm)/TmPyPB(50nm)/LiF } \\
(1 \mathrm{~nm}) / \mathrm{Al}(100 \mathrm{~nm})\end{array}$ & 3.1 & 29 & 81.6 & 71.1 & $0.39,0.46$ & [66] \\
\hline W-D37 & $\begin{array}{l}\text { ITO/HAT-CN }(10 \mathrm{~nm}) / \mathrm{TAPC}(40 \mathrm{~nm}) / \mathrm{TCTA}(10 \mathrm{~nm}) / \mathrm{H} 12: \\
\text { Flrpic } 15 \%: \text { PO-01 1\%(20nm)/TmPyPB }(50 \mathrm{~nm}) / \mathrm{Liq}(2 \\
\mathrm{nm}) / \mathrm{Al}(120 \mathrm{~nm})\end{array}$ & - & 27.8 & 75 & 64.2 & $0.40,0.46$ & [72] \\
\hline W-D38 & $\begin{array}{l}\text { ITO/TAPC }(30 \mathrm{~nm}) / \mathrm{TCTA}(5 \mathrm{~nm}) / \mathrm{mCP}(5 \mathrm{~nm}) / \mathrm{H} 19: \mathrm{PO}-01 \\
0.4 \%(20 \mathrm{~nm}) / \mathrm{TPYMB}(50 \mathrm{~nm}) / \mathrm{LiF}(1 \mathrm{~nm}) / \mathrm{Al}(100 \mathrm{~nm})\end{array}$ & 2.6 & 27.5 & 84.0 & 94.8 & $0.45,0.46$ & [84] \\
\hline W-D39 & $\begin{array}{l}\text { ITO/HAT-CN(10nm)/TAPC(45 nm)/H24:FIrpic:PO-01 } \\
(15 \%, 1 \%, 20 \mathrm{~nm}) / \mathrm{TmPyPB}(40 \mathrm{~nm}) / \mathrm{Liq}(2 \mathrm{~nm}) / \mathrm{Al}\end{array}$ & 4.3 & 27.2 & 53.2 & 44.4 & & [87] \\
\hline W-D40 & $\begin{array}{l}\text { ITO/TAPC(40nm)/H18:FIrpic:PO-01(100:23:0.7,10nm) } \\
\text { TmPyPB (40nm)/LiF(1nm)/Al(100nm) }\end{array}$ & 2.7 & 26.4 & 74.3 & 82.7 & $0.32,0.46$ & [83] \\
\hline
\end{tabular}

Tableau 6. Exemples de performances de dispositifs PhOLEDs à émission blanche

$H 12$ [72] avec un $E_{T}$ de $2.81 \mathrm{eV}$ a été présenté ci-dessus comme matrice hôte pour le dopant vert $\operatorname{Ir}(\mathrm{ppy})_{3}$ dans $\mathrm{D} 20$ avec un EQE $\mathrm{E}_{\max }$ de plus de 33 \%.[72] Les performances avec le dopant bleu FIrpic sont un peu plus faibles ( $21.4 \%$ ) mais elles montrent que $\mathbf{H 1 2}$ peut être utilisé comme matrice hôte pour le FIrpic et Ir $(\mathrm{ppy})_{3}$, mais peut aussi être co-dopé par Flrpic et PO-01. La diode W-D37 atteint un EQE de $27.8 \%$ et émet une lumière blanche de coordonnées chromatiques $(0.40,0.46)$.

Les matrices $\mathbf{H 2 4}$ [87] ( $\left.E_{\mathrm{T}}: 2.8 \mathrm{eV}\right)$ et $\mathbf{H 1 8}$ [83] $\left(\mathrm{E}_{\mathrm{T}}: 3.02 \mathrm{eV}\right)$ sont aussi des matrices universelles et lorsqu'elles sont co-dopées par Flrpic et $\mathrm{PO}-01$, elles émettent une lumière blanche avec des performances remarquable (EQE $\left.E_{\max }>26.4 \%\right)$ pour W-D39 et W-D40.

La diode W-D38 utilise comme matrice hôte $\mathbf{H 1 9}$ [84] ( $\mathrm{E}_{\mathrm{T}}$ : $\left.2.35 \mathrm{eV}\right)$ et comme dopant PO-01. H19 n'est pas une matrice adaptée au dopant bleu FIrpic du fait de son $E_{T}$ trop bas en énergie. Utilisée dans D28 comme matrice hôte pour PO-01 avec un taux de dopage de $3 \%$ en masse, elle émet une lumière jaune-orange $(0.51,0.48)$. Dans W-D38, le taux de dopage de $\mathrm{PO}-01$ dans $\mathbf{H} 19$ est diminué à $0.4 \%$. L'émission observée est une émission blanche, $\mathrm{CIE}(0.45,0.46)$, composée de l'émission jauneorange par phosphorescence du dopant $\mathrm{PO}-01$ et de l'émission bleue par fluorescence de la matrice hôte H19. Cette OLED phosphorescente hybride a un EQEmax dépassant $27 \%$ et un $V_{\text {on }}$ similaire à celui de la diode D28 (2.6 V) montrant que l'injection des charges se fait de façon similaire dans les deux dispositifs et que leur combinaison est moins efficace lorsque le taux de dopage de PO-01 augmente dans la matrice hôte. On notera dans la diode multicouche W-D38, la présence entre l'EML et la cathode d'une couche de tris(2,4,6-triméthyl-3-(pyridin-3-yl)phényl)borane (TPYMB) jouant le rôle d'EIL, ETL et HBL.

\section{Conclusion}

Les PhOLEDs ont connu en 20 ans un développement fantastique et atteignent aujourd'hui des performances remarquables. Ceci est dû à la synergie entre chimie et physique au travers du design des matrices et celui des dispositifs.[4] Les règles de design des matrices sont aujourd'hui connues et 
les performances des PhOLEDs continuent d'augmenter dans toutes les couleurs.[56] Malgré ces avancées spectaculaires, les caractéristiques des diodes phosphorescentes ne remplissent pas parfaitement et pour toutes les couleurs, le cahier des charges. En effet, les diodes phosphorescentes utilisent des dopants à base d'iridium (voire de platine...) qui sont des composés onéreux et de ressources épuisables. Un autre obstacle est lié à l'émission de lumière bleue en particulier qui (i) nécessite la synthèse de matrices hôtes à haut état triplet, avec pour corollaire un SCO à état singulet encore plus élevé ce qui n'est pas simple à mettre en forme et (ii) n'est pas suffisamment stable. Les PhOLEDs représentent cependant la technologie la plus mature en 2020.

Face à ces limitations et suite aux premiers travaux du groupe d'Adachi en 2009,[21] les chercheurs ont commencé à développer un nouveau type d'OLEDs basé sur la fluorescence retardée activée thermiquement, que nous allons décrire maintenant et qui n'utilise pas de complexes métalliques.

\section{À retenir}

- Dans une PhOLED :

- l'émission provient d'un dopant phosphorescent présent en faible quantité dans une matrice organique hôte dans laquelle sont injectés les électrons et les trous.

- la couleur de la lumière émise est celle de la lumière émise par le dopant phosphorescent.

- L'IQE peut atteindre $100 \%$ si tous les excitons (singulet et triplet) générés dans la matrice organique hôte sont transférés au dopant phosphorescent, $\mathrm{E}_{\mathrm{T}}$ matrice hôte > ET dopant.

- L'EQE peut dépasser $30 \%$ selon l'efficacité du découplage de la lumière dans la diode

\section{Les OLEDs à fluorescence retardée activée thermiquement}

En 2009, le groupe d'Adachi[21] (Kyushu, Japon) a mis en évidence un nouveau mécanisme d'émission, basé sur la fluorescence retardée activée thermiquement, permettant de récupérer l'ensemble des excitons (singulets et triplets) formés dans la couche émissive de l'OLED. Les OLEDs TADF (pour Thermally Activated Delayed Fluorescence) permettent de s'affranchir des émetteurs phosphorescents à base de platine ou d'iridium tout en atteignant des rendements d'émission élevés.

\subsection{Principe de fonctionnement d'une OLED TADF}

Qu'est-ce que la fluorescence retardée activée thermiquement ? Dans un émetteur TADF, les niveaux $S_{1}$ et $T_{1}$ sont fortement couplés autorisant un croisement intersystème entre eux. Ainsi, la molécule est construite de façon à ce que la différence d'énergie entre ces deux niveaux $\left(\Delta \mathrm{E}_{\mathrm{ST}}=\mathrm{E}_{\mathrm{S}_{1}}-\mathrm{E}_{T 1}\right)$ soit la plus faible possible (de préférence $<0.1 \mathrm{eV}$ )[88]. En effet, ce faible écart énergétique permet un croisement intersystème inverse (RISC pour Reverse InterSystem Crossing) autorisant une conversion des excitons triplets formés dans la molécule TADF (75 \%) en excitons singulets dans un processus activé thermiquement.

Le principe de fonctionnement d'une OLED TADF est donc le suivant (Figure 10). Comme pour les OLEDs fluorescentes et phosphorescentes, lors de la mise sous tension de l'OLED TADF, des électrons et des trous provenant des électrodes sont injectés dans le SCO, ils se combinent pour former les excitons dans un rapport de 25/75 d'excitons singulets/triplets.

Rapidement, l'émission de fluorescence à partir des excitons singulets du SCO est observée, elle est nommée PF (pour « prompt fluorescence »). Dans le même temps, les excitons triplets du SCO TADF sont transformés en excitons singulets grâce au RISC. Ces excitons singulets émettent alors de la fluorescence retardée DF (pour Delayed Fluorescence). Au bilan, 100 \% des excitons formés dans le 
SCO TADF se retrouvent à l'état d'excitons singulets et reviennent à l'état fondamental en émettant de la lumière (retour de $S_{1}$ vers $S_{0}$ ) soit par fluorescence rapide PF (généralement de l'ordre de la nanoseconde) soit par fluorescence retardée DF (généralement de l'ordre de la microseconde). La couleur de la lumière émise est donc celle du SCO.

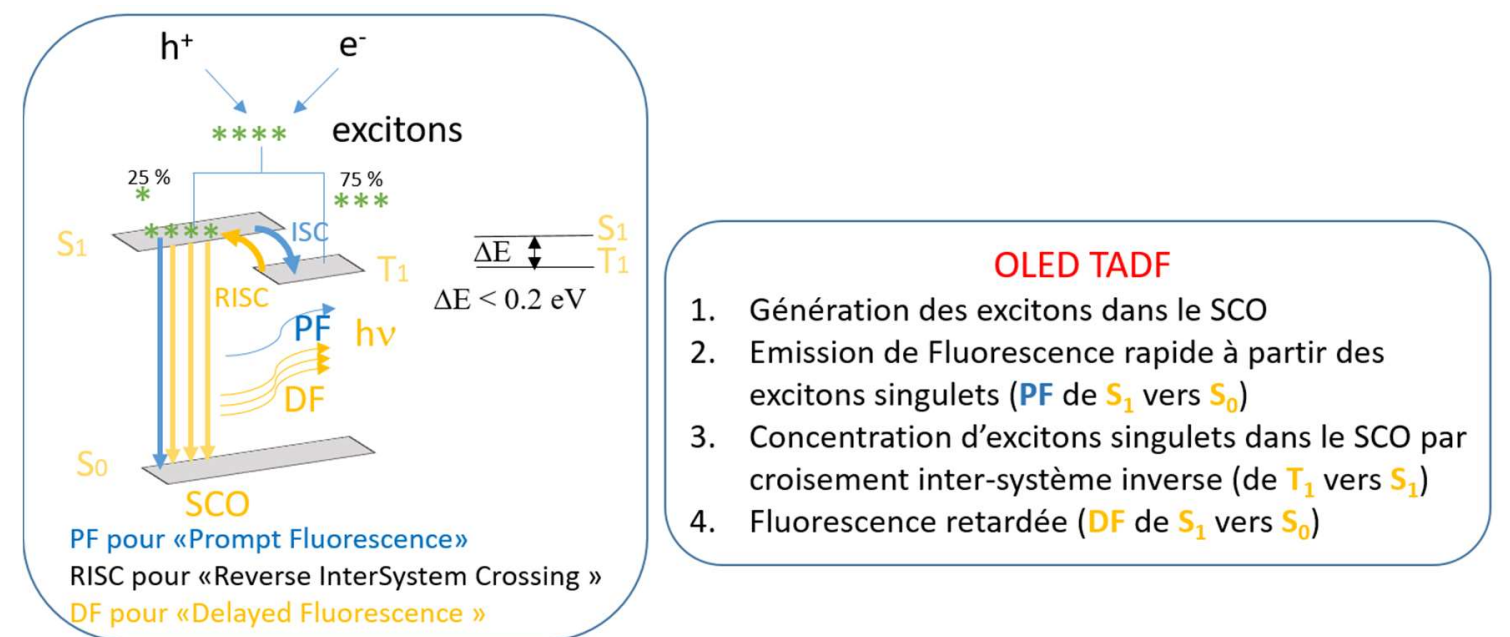

Figure 10. Principe de fonctionnement d'une OLED TADF

Les propriétés requises pour qu'un SCO puisse être utilisé comme couche active d'une OLED TADF sont similaires à celles décrites pour le SCO d'une OLED fluorescente pour les items (i)-(iv) (voir page $4 / 5$ ) avec cette fois une contrainte supplémentaire concernant l'écart entre les niveaux d'énergie $S_{1}$ et $T_{1}\left(\Delta \mathrm{E}_{S T}\right)$ dans le SCO TADF. Il faut que $\Delta \mathrm{E}_{S \mathrm{ST}}$ soit le plus faible possible (de préférence $<0.1 \mathrm{eV}$ ) [88]. Ceci peut être notamment obtenu par une séparation spatiale de la HOMO et de la LUMO du SCO qui minimise les interactions entre les deux orbitales.

La séparation spatiale des orbitales HOMO et LUMO dans le SCO peut être obtenue par exemple lorsqu'un accepteur d'électrons (A) et un donneur d'électrons (D) sont reliés via les positions méta d'une unité phényle (découplage électronique) ou lorsqu'un angle de torsion important entre $A$ et $D$ vient empêcher le recouvrement des orbitales. L'utilisation d'atome «isolant» comme l'atome de phosphore ou l'utilisation de géométrie moléculaire particulière comme la géométrie "spiro» sont aussi très fréquentes.[23, 24, 89, 90]

Déterminer expérimentalement $\Delta \mathrm{E}_{\mathrm{ST}}$ est donc crucial dans ces technologies. Les valeurs que l'on trouve dans la littérature doivent être regardées avec précaution. En effet, le plus souvent, $\Delta \mathrm{E}_{S \mathrm{ST}}$ est déterminé par la différence d'énergie (en eV) entre la longueur d'onde du seuil d'émission de fluo-

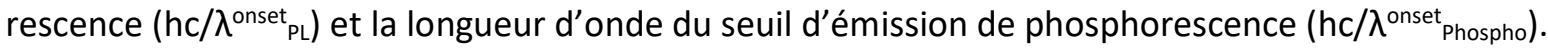
Dans le cas de la fluorescence, la mesure peut être faite à température ambiante ou à basse température (77K ou même un peu plus bas), ce qui peut entraîner des différences notables. D'autre part, les maxima d'émission de fluorescence et de phosphorescence sont aussi souvent utilisés à la place des seuils. Ces différences expérimentales lors des mesures entrainent des différences importantes dans la détermination du $\Delta \mathrm{E}_{\mathrm{ST}}$ qui rend la comparaison des données présentées dans la littérature très difficiles. Un regard critique doit toujours être porté sur toutes ces valeurs. $\Delta \mathrm{E}_{S T}$ peut aussi être obtenu à partir des calculs théoriques de $S_{1}$ et $T_{1}$.

\subsection{Emission bleue des OLEDs TADF}

Le tableau 7 rassemble les performances de diodes dont l'EML est composée (i) du SCO TADF pur appelées " OLED TADF non dopées » (D41) ou (ii) du SCO TADF utilisé comme dopant d'une matrice hôte appelées « OLED TADF dopées » (D42-D44) dont les différents composants sont présentés Figure 11.

Dans une " OLED-TADF non dopée ", le SCO TADF utilise les excitons triplets dans le processus conduisant à l'émission et du fait de la plus longue durée de vie de ces excitons triplets, ces derniers sont facilement perturbés par différents facteurs extérieurs comme la désactivation par effet de concentration, l'annihilation triplet-triplet, la migration des excitons,...[91] ce qui diminue l'efficacité du dispositif.[88] La dispersion du SCO TADF dans une matrice hôte diminue les interactions entre les 
excitons triplets et améliore l'efficacité des " OLEDs TADF dopées » par rapport à celle des « OLEDs TADF non dopées $"$.

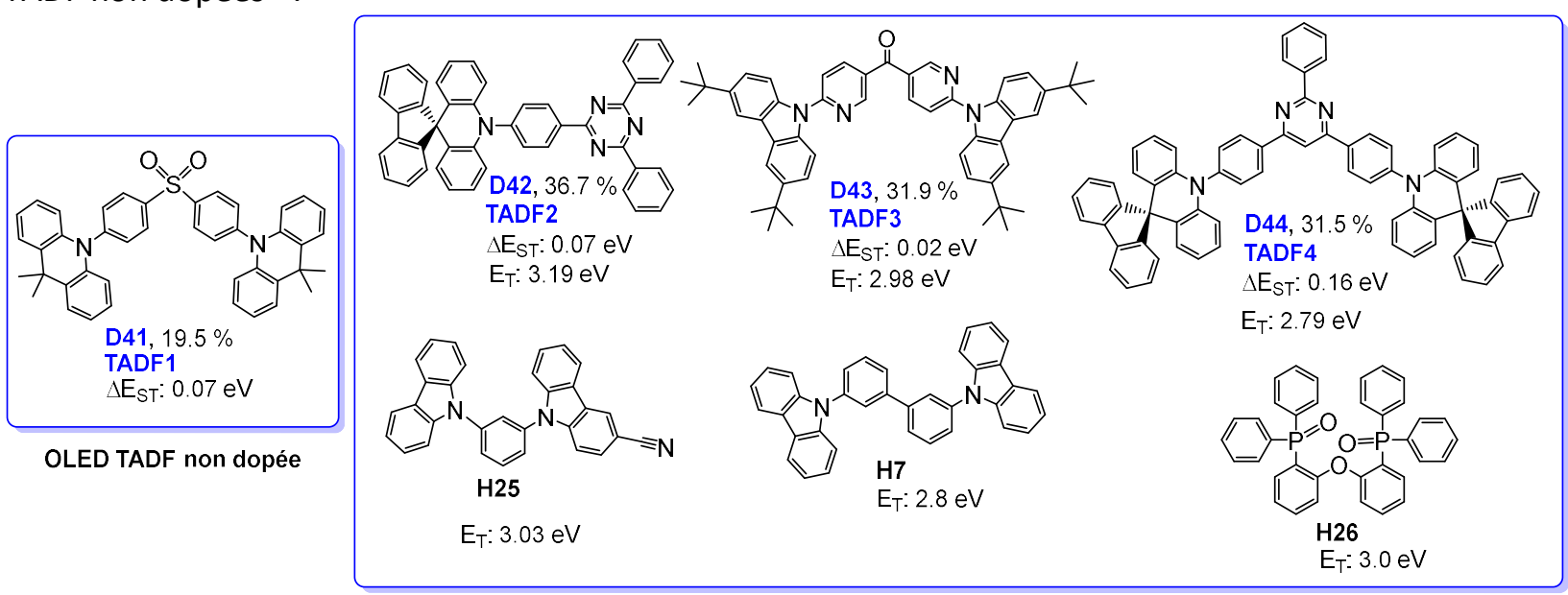

Couples matrice hôte/dopant TADF bleu utilisés dans les OLEDs TADF dopées D42-D44

Figure 11. Exemples de SCOs à propriété TADF pour une émission bleue

Dans D41, la couche émissive est constituée de TADF1 qui possède une architecture D-A-D avec I'unité accepteur d'électrons diphénylsulfone et les unités donneuses d'électrons 9,9-diméthyl-9,10dihydroacridine.[92] Le dispositif atteint un $\mathrm{EQE}_{\max }$ supérieur à $19 \%$, la lumière émise est bleue $\left(\lambda_{\max }: 480 \mathrm{~nm}, \mathrm{ClE}: 0.16,0.29\right)$. Les performances de D41 sont très largement supérieures à celles des OLEDs fluorescentes bleues les plus efficaces (D2 et D3, EQE proche de 7\%) montrant clairement l'efficacité de la fluorescence retardée. Ces performances restent cependant inférieures à celles des meilleures PhOLEDs bleues (D10-D15, EQE compris entre 33.2 et $24.7 \%$ ).

\begin{tabular}{|c|c|c|c|c|c|c|c|c|}
\hline & Architecture du dispositif & $\begin{array}{l}V_{\text {on }} \\
\text { (V) }\end{array}$ & $\begin{array}{c}\text { EQE }_{\max } \\
(\%)\end{array}$ & $\begin{array}{l}\mathrm{CE}_{\max } \\
(\mathrm{cd} / \mathrm{A})\end{array}$ & $\begin{array}{c}\mathrm{PE}_{\max } \\
(\mathrm{Im} / \mathrm{W})\end{array}$ & $\begin{array}{l}\lambda_{\max } \\
(\mathrm{nm})\end{array}$ & $\begin{array}{c}\text { CIE } \\
(x, y)\end{array}$ & Réf. \\
\hline \multicolumn{9}{|c|}{ Dispositif TADF-OLED multicouche bleu } \\
\hline D41 & $\begin{array}{l}\text { ITO/MoO }(1 \mathrm{~nm}) / \mathrm{mCP}(40 \mathrm{~nm}) / \mathrm{TADF} 1(30 \mathrm{~nm}) / \mathrm{H} 26(50 \mathrm{~nm}) / \\
\mathrm{LiF}(1 \mathrm{~nm}) / \mathrm{Al}\end{array}$ & 4.3 & 19.5 & - & - & 480 & $0.16,0.29$ & [92] \\
\hline D42 & $\begin{array}{l}\text { ITO/MoO }(1 \mathrm{~nm}) / \mathrm{TAPC}(50 \mathrm{~nm}) / \mathrm{mCP}(10 \mathrm{~nm}) / \mathrm{H} 25: \mathrm{TADF}(12 \\
\%)(20 \mathrm{~nm}) / \mathrm{TPYMB}(50 \mathrm{~nm}) / \mathrm{LiF}(0.5 \mathrm{~nm}) / \mathrm{Al}(150 \mathrm{~nm})\end{array}$ & 2.8 & 36.7 & 94.0 & 98.7 & 490 & $0.18,0.43$ & [93] \\
\hline D43 & $\begin{array}{l}\text { ITO/NPB(30nm)/TAPC }(20 \mathrm{~nm}) / \mathrm{H7}(10 \mathrm{~nm}) / \mathrm{H7}: \mathrm{TADF} 3(7 \%) \\
(30 \mathrm{~nm}) / \mathrm{H} 26(5 \mathrm{~nm}) / \mathrm{TmPyPB}(60 \mathrm{~nm}) / \mathrm{LiF}(0.8 \mathrm{~nm}) / \mathrm{Al}(100 \mathrm{~nm})\end{array}$ & 3.7 & 31.9 & 37.6 & 37.3 & 464 & $0.14,0.18$ & [94] \\
\hline D44 & $\begin{array}{l}\text { ITO/HAT-CN }(5 \mathrm{~nm}) / \text { TAPC }(30 \mathrm{~nm}) / \mathrm{mCP}(10 \mathrm{~nm}) / \mathrm{H} 26: \mathrm{TADF} 430 \% \\
(20 \mathrm{~nm}) / \mathrm{H} 26(5 \mathrm{~nm}) / \mathrm{TPBI}(40 \mathrm{~nm}) / \mathrm{LiF}(0.9 \mathrm{~nm}) / \mathrm{Al}(100 \mathrm{~nm})\end{array}$ & 3.6 & 31.5 & 68.8 & 56.9 & - & $0.18,0.32$ & [95] \\
\hline
\end{tabular}

Tableau 7. Exemples de performances de dispositifs OLED-TADF à émission bleue

Dans D42 [93], le SCO d'architecture D- $\pi-A$, TADF2, est utilisé comme dopant dans une matrice de 9(3-(9H-carbazol-9-yl)phenyl)-9H-carbazole-3-carbonitrile (H25) qui est une matrice bipolaire à $\mathrm{E}_{\mathrm{T}} 3.03 \mathrm{eV}$ et gap HOMO/LUMO $3.56 \mathrm{eV}$ (HOMO : -5.8 eV, LUMO : -2.24 eV).[96] TADF2 est formé d'une unité donneur de type spirofluorène-acridine et d'une unité accepteur dérivée de triazine reliées par un phényle. La structure RX de ce SCO montre un angle de $90^{\circ}$ entre les deux unités $D$ et $A$ entrainant une séparation spatiale des orbitales moléculaires (la HOMO étant localisée sur l'unité donneur et la LUMO sur l'unité accepteur). La valeur de $\triangle \mathrm{E}_{S T}$ de TADF2 a été déterminée à $0.07 \mathrm{eV}$ à partir des spectres d'émission de fluorescence enregistré à $300 \mathrm{~K}$ et de phosphorescence enregistré à $77 \mathrm{~K}$ d'un film de $\mathbf{H 2 5}$ dopé à $12 \%$ en masse par TADF2. D42 émet dès $2.8 \mathrm{~V}$ une lumière bleu-vert de coordonnées $\mathrm{CIE}(0.18,0.43)$ avec un EQE $\mathrm{E}_{\max }$ atteignant $36.7 \%$. Cette diode à émetteur TADF est plus efficace que la meilleure PhOLED bleue (D10) avec une augmentation de l'EQE max $_{\text {ax }}$ de $33.2 \%$ pour D10 à $36.7 \%$ pour D42. Ce résultat est très important car il montre que les OLEDs TADF peuvent dépasser les PhOLEDs en terme d'efficacité.

Dans D43, le SCO TADF3 qui présente un $\triangle \mathrm{E}_{S T}$ de $0.02 \mathrm{eV}$ est utilisé comme dopant de la matrice hôte $\mathbf{H 7}\left(\mathrm{E}_{\mathrm{T}}: 2.8 \mathrm{eV}\right)$.[94] Cette matrice hôte possède un gap HOMO/LUMO (-6.0/-2.4 eV) plus large que celui de $\mathrm{H} 25$ utilisé dans D42 entrainant une augmentation du $\mathrm{V}_{\text {on }}$ de $2.8 \mathrm{~V}$ pour D42 à $3.7 \mathrm{~V}$ 
pour D43. L'EQE $E_{\max }$ de D43 est un peu plus faible (31.9 vs $36.7 \%$ ) que celui de D42 tout en restant très élevé. Le spectre d'EL de D43 montre l'émission unique de TADF3 indiquant une bonne cascade d'énergie entre la matrice hôte et le dopant. A ce titre, le spectre d'EL de D43 est centré à $464 \mathrm{~nm}$ avec des coordonnées chromatiques $(0.14,0.18)$ indiquant un bleu plus profond que celui émis par les deux diodes précédentes. Les performances de D43 comparées à celles de la PhOLED D15 émettrice d'un bleu profond $(0.13,0.13)$ sont supérieures avec une augmentation d'un tiers de l'EQE $_{\max }$ (31.9 vs $24.7 \%$ ).

Dans D44, la matrice hôte est l'oxyde de bis[2-(diphénylphosphino)phényl]éther (H26).[95] Cette matrice hôte à large gap HOMO/LUMO (4.1 eV) et $\mathrm{E}_{\mathrm{T}}$ élevé $(3.0 \mathrm{eV})$ est souvent utilisée dans les OLED TADF.[97] TADF4 a une architecture D-A-D avec une unité accepteur d'électrons phénylpyrimidine liée à deux unités donneurs d'électron phénylacridine via deux ponts phényles. L'EML de D44 est composée de H26 dopé par TADF4. D44 atteint un EQE $E_{\max }$ de $31.5 \%$ avec une émission bleu-clair issue de TADF4 (CIE : 0.18, 0.32). Ces performances montrent que la cascade de transfert énergétique se fait efficacement du fait du faible $\Delta \mathrm{E}_{S T}$ de TADF4 $(0.16 \mathrm{eV})$ entrainant un processus RISC efficace. D'autre part, l'adéquation des niveaux HOMO/LUMO de la matrice hôte et du dopant est toujours un point clé dans l'efficacité de l'OLED.

\subsection{Emission verte des OLEDs TADF}

La diode TADF non dopée D45, multicouche, utilise comme EML le SCO TADF5 pur (Figure 12, Tableau 8).[92] TADF5 possède, comme TADF1, une architecture D-A-D avec une unité accepteur d'électrons plus forte, la benzophénone et les unités donneuses d'électrons 9,9-diméthyl-9,10-dihydroacridine. L'émission en film mince de TADF5 est centrée à $506 \mathrm{~nm}$ (couleur verte) décalée par rapport à celle d'un film mince de TADF1 ( $470 \mathrm{~nm}$, couleur bleue). Le $\triangle \mathrm{E}_{\text {ST }}$ de TADF5 est de $0.07 \mathrm{eV}$, similaire à celui de TADF1, laissant présager de bonnes propriétés TADF. D44 atteint un $\mathrm{EQE}_{\max }$ de $18.9 \%$ dépassant très largement les performances des OLEDs à émetteur fluorescent vert classique (D6, EQE $E_{\max }: 4.4 \%$ ).

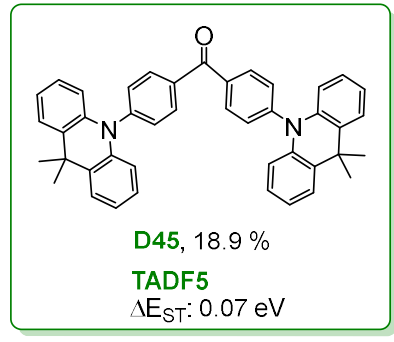

OLED TADF non dopée
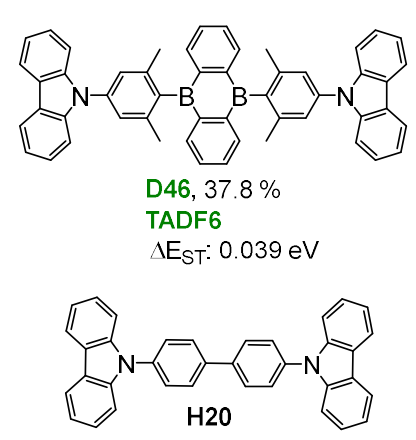

$\mathrm{E}_{\mathrm{T}}: 2.6 \mathrm{eV}$

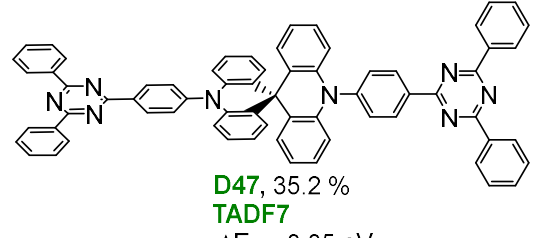

$\Delta \mathrm{E}_{\mathrm{ST}}: 0.05 \mathrm{eV}$

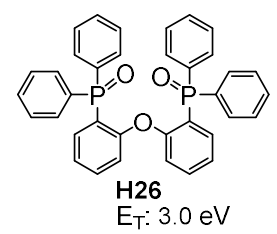

Couples matrice hôte/dopant TADF vert utilisé dans les OLEDs TADF dopées D46 et D47

Figure 12. Exemples de SCOs à propriété TADF pour une émission verte

\begin{tabular}{|c|c|c|c|c|c|c|c|c|}
\hline & Architecture du dispositif & $\begin{array}{l}\mathrm{V}_{\text {on }} \\
(\mathrm{V})\end{array}$ & $\begin{array}{c}\mathrm{EQE}_{\max } \\
(\%)\end{array}$ & $\begin{array}{l}\mathrm{CE}_{\max } \\
(\mathrm{cd} / \mathrm{A}) \\
\end{array}$ & $\begin{array}{l}\mathrm{PE}_{\max } \\
(\mathrm{Im} / \mathrm{W})\end{array}$ & $\begin{array}{c}\lambda_{\max } \\
(\mathrm{nm})\end{array}$ & $\begin{array}{c}\text { CIE } \\
(x, y)\end{array}$ & Réf. \\
\hline \multicolumn{9}{|c|}{ Dispositif TADF-OLED multicouche verte } \\
\hline D45 & $\begin{array}{l}\text { ITO/MoO }(1 \mathrm{~nm}) / \mathrm{mCP}(40 \mathrm{~nm}) / \mathrm{TADF} 5(30 \mathrm{~nm}) / \mathrm{TPBI} \\
(50 \mathrm{~nm}) / \mathrm{LiF}(1 \mathrm{~nm}) / \mathrm{Al}\end{array}$ & 2.6 & 18.9 & & & 510 & $0.26,0.55$ & [92] \\
\hline D46 & $\begin{array}{l}\text { ITO/NPB(40nm)/TCTA(10nm)/H20:TADF6 10\% } \\
(30 \mathrm{~nm}) / \mathrm{TmPyPB}(60 \mathrm{~nm}) / \mathrm{LiF}(1 \mathrm{~nm}) / \mathrm{Al}(100 \mathrm{~nm})\end{array}$ & 2.7 & 37.8 & 139.6 & 121.6 & 528 & $0.31,0.61$ & [98] \\
\hline D47 & $\begin{array}{l}\text { ITO/polymère à base de triphénylamine dopé au } \\
\mathrm{PPBI}(20 \mathrm{~nm}) / \mathrm{TAPC}(20 \mathrm{~nm}) / \mathrm{mCP}(10 \mathrm{~nm}) / \mathrm{H} 26: \mathrm{TADF} 7 \\
20 \%(20 \mathrm{~nm}) / \mathrm{H} 26(10 \mathrm{~nm}) / \mathrm{BmPyPB}(40 \mathrm{~nm}) / \mathrm{LiF} \\
(0.5 \mathrm{~nm}) / \mathrm{Al}\end{array}$ & 3.1 & 35.2 & 98 & 90.5 & & $0.24,0.48$ & [99] \\
\hline
\end{tabular}

Tableau 8. Exemples de performances de dispositifs OLED TADF à émission verte

Les diodes TADF dopées D46-47, sont des diodes multicouches à émetteur TADF6[98] ou TADF7[99] dopant respectivement les matrices hôtes $\mathbf{H 2 0}$ ou $\mathbf{H 2 6}$ présentées précédemment ( $\mathbf{H 2 0}$ : figure 8 et H26 : figure 11). TADF6 possède une structure de type D-A-D avec deux unités carbazole donneur 
d'électron liées chacune par un pont ortho-diméthylphényle à une unité centrale diboroanthracène (DBA) accepteur d'électrons. Ces deux ponts, non seulement protège l'unité centrale DBA, mais aussi, réduisent la conjugaison dans TADF6 et séparent la HOMO localisée sur les unités carbazole et la LUMO localisée sur l'unité DBA. Dans TADF6, seules les unités carbazoles peuvent tourner autour de la liaison $\mathrm{N}-\mathrm{C}$ alors que la partie centrale de la molécule est totalement figée. Les propriétés TADF de TADF6 ont été mises en évidence dans des films de $\mathbf{H 2 0}$ à différentes températures (de 77 à 300 K) montrant une émission de fluorescence rapide avec une durée de vie de 22 ns et une émission retardée de durée de vie $3.2 \mu \mathrm{s}$. La diode D46 dans laquelle l'EML est TADF6 dopant une matrice de H2O présente un spectre d'EL centré à $528 \mathrm{~nm}$ (vert-jaunâtre, CIE : 0.31, 0.61) provenant de l'émission de TADF6 sans émission de la matrice hôte ni des couches intermédiaires. L'EQE $E_{\max }$ est extrêmement élevé et atteint $37.8 \%$.

TADF7 est un émetteur TADF vert de type A-D-spiro-D-A. Les groupements accepteurs d'électrons externes de type diphényl-triazine (TZ) sont reliés aux groupes donneurs d'électrons de type phénylacridine eux-mêmes reliés entre eux par un atome de carbone spiro. Les deux unités acridines sont donc orientées perpendiculairement l'une à l'autre dans une géométrie rigide induite par le pont spiro. D'autre part, des interactions azote-hydrogène entre les unités triazines et le phényle adjacent entrainent un blocage de la rotation entre les unités TZ et le phényle ajoutant une rigidité supplémentaire à la molécule. Le faible écart $\Delta \mathrm{E}_{S \mathrm{ST}}(0.05 \mathrm{eV})$ et les mesures de photoluminescence de TADF7 dans des films de $\mathbf{H 2 6}$ à différentes températures (de 5 à $300 \mathrm{~K}$ ) ont permis de mettre en évidence l'émission de fluorescence rapide et l'émission retardée de durée de vie $9.6 \mu$ s. La diode D47 dans laquelle l'EML est une matrice de H26 dopée par $20 \%$ de TADF7 atteint un EQE de $35.2 \%$ et émet une lumière verte $(\mathrm{CIE}: 0.24,0.48)$ provenant uniquement de TADF7. On notera dans D47, I'utilisation d'une couche de polymère à base de triphénylamine dopé par du 4-isopropyl-4'méthyldiphényliodonium tetrakis(pentafluorophényl)borate (PPBI) comme couche d'HIL.

Ces deux exemples montrent qu'une architecture étendue (mais présentant malgré tout une rupture de conjugaison centrale point clé pour maintenir de hauts $E_{S} / E_{T}$ ) et rigide du dopant TADF favorise une orientation du SCO dans la matrice hôte permettant une bonne mobilité des charges et conduisant à des dispositifs très efficaces. Dans les deux cas, cette efficacité dépasse largement celle des OLED fluorescentes vertes classiques (D4-D6) mais aussi celle des PhOLEDs (D20-D23).

\subsection{Emission jaune/orange des OLEDs TADF}

Dans la diode non dopée D48, [100] TADF6 est le seul composant de l'EML (Figure 13, Tableau 9). $\mathrm{D} 48$ émet, dès $2.1 \mathrm{~V}$, une lumière jaune-orangée centrée à $560 \mathrm{~nm}$ avec un $E Q E_{\max }$ de $19 \%$. D48 est décrite par les auteurs comme une diode stable et à longue durée de vie. On notera que TADF6 a été utilisé précédemment (tableau 8) comme dopant de $\mathbf{H 2 0}$ dans une OLED d'architecture différente D46. D46 émet, dès $2.7 \mathrm{~V}$, une lumière verte centrée à $528 \mathrm{~nm}$ et atteint un EQE de $37.8 \%$. Le décalage de la longueur d'onde d'émission entre D46 et D48 est en accord avec l'émission d'agrégats dans D48 et l'émission de la molécule isolée dans D46.

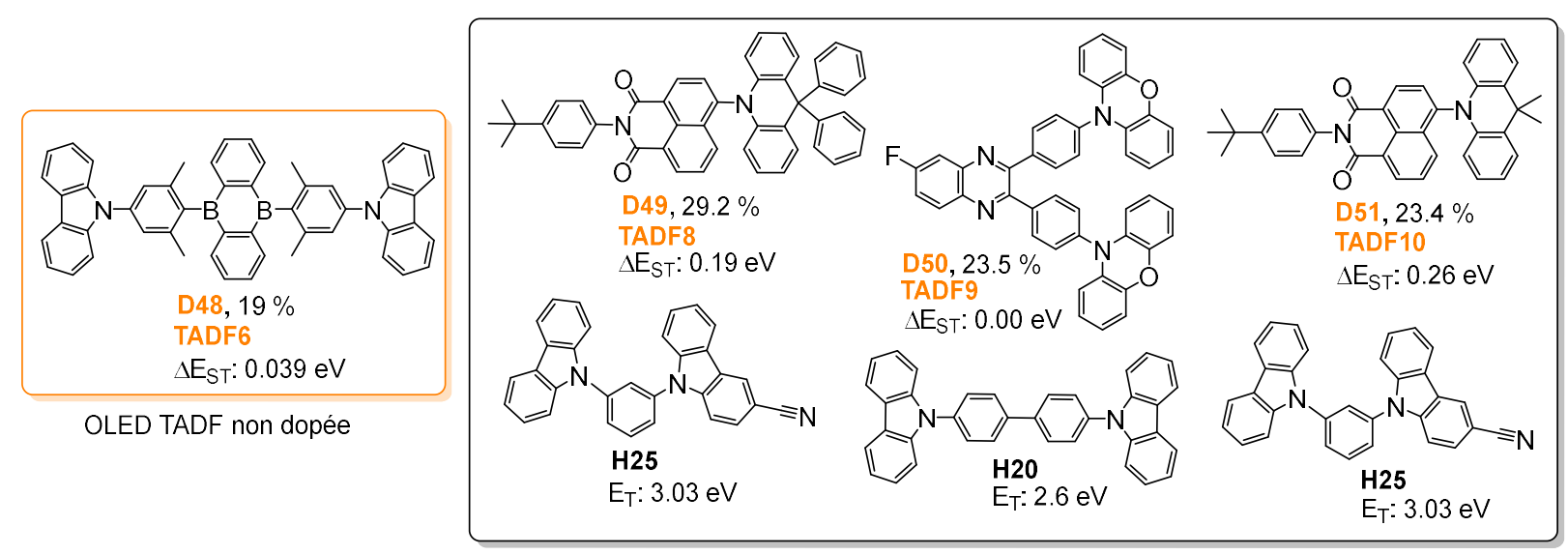

Couples matrice hôte/dopant jaune-orange utilisés dans les OLEDs TADF dopées D49-D51

Figure 13. Exemples de SCOs à propriété TADF pour une émission jaune/orange 


\begin{tabular}{|c|c|c|c|c|c|c|c|}
\hline & Architecture du dispositif & $\begin{array}{l}\text { Von } \\
\text { (V) }\end{array}$ & $\begin{array}{c}\text { EQEmax }^{(\%)} \\
\text { (\%) }\end{array}$ & $\begin{array}{c}C E_{\max } \\
(\mathrm{cd} / \mathrm{A})\end{array}$ & $\begin{array}{c}P E_{\max } \\
(\mathrm{Im} / \mathrm{W})\end{array}$ & $\begin{array}{l}\lambda_{\max } \\
(\mathrm{nm})\end{array}$ & Réf. \\
\hline \multicolumn{8}{|c|}{ Dispositif TADF-OLED multicouche jaune-orange } \\
\hline D48 & 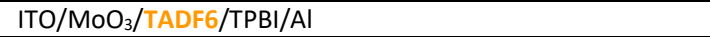 & 2.1 & 19 & - & 87 & 560 & {$[100]$} \\
\hline D49 & $\begin{array}{l}\text { ITO/MoO } 3(2 \mathrm{~nm}) / \mathrm{TAPC}(70 \mathrm{~nm}) / \mathrm{mCP}(10 \mathrm{~nm}) / \mathrm{H} 25: \text { TADF8 6\% } \\
(20 \mathrm{~nm}) / \mathrm{TPYMB}(70 \mathrm{~nm}) / \mathrm{LiF}(0.5 \mathrm{~nm}) / \mathrm{Al}\end{array}$ & 3.0 & 29.2 & 76.2 & 79.7 & 584 & {$[101]$} \\
\hline D50 & $\begin{array}{l}\text { ITO/MoO }{ }_{3}(10 \mathrm{~nm}) / \mathrm{TAPC}(50 \mathrm{~nm}) / \mathrm{mCP}(10 \mathrm{~nm}) / \mathrm{H2O}: \text { TADF9 10\% } \\
(20 \mathrm{~nm}) / \mathrm{TPYMB}(70 \mathrm{~nm}) / \mathrm{LiF}(0.5 \mathrm{~nm}) / \mathrm{Al}\end{array}$ & 2.7 & 23.5 & 78.3 & 91.1 & 548 & [102] \\
\hline D51 & $\begin{array}{l}\text { ITO/MoO }(2 \mathrm{~nm}) / \mathrm{TAPC}(70 \mathrm{~nm}) / \mathrm{mCP}(10 \mathrm{~nm}) / \mathrm{H} 25: \mathrm{TADF} 106 \% \\
(20 \mathrm{~nm}) / \mathrm{TPYMB}(70 \mathrm{~nm}) / \mathrm{LiF}(0.5 \mathrm{~nm}) / \mathrm{Al}\end{array}$ & 3.0 & 23.4 & 50.7 & 53.1 & 597 & {$[101]$} \\
\hline
\end{tabular}

Tableau 9. Exemples de performances de dispositifs OLED TADF à émission jaune/orange (D48-D51)

TADF8 et TADF10 [101] ont tous les deux une architecture D-A présentant une unité N-(4-tertbutylphényl)-1,8-naphtalimide (NAI) comme unité accepteur d'électron et une unité donneur d'électrons dérivée de l'acridine. NAI est choisi comme accepteur d'électrons du fait de sa structure plane et rigide et de son fort pouvoir accepteur. Utilisé comme dopant de $\mathbf{H 2 5}$, les deux composés conduisent à des OLED-TADF d'EQE élevé (29.2\% pour D49 et $23.4 \%$ pour D51) et émettent une lumière orange centrée à $584 \mathrm{~nm}$ pour D49 et $597 \mathrm{~nm}$ pour D51.

Les performances de D50 [102], utilisant TADF9 comme dopant de H20 se situent entre celles de D49 et de D51. TADF9 est basé sur une quinoxaline accepteur d'électrons et deux unités phénothiazine donneurs d'électrons. Un atome de fluor substitue l'unité quinoxaline dans le but de favoriser le couplage électronique intermoléculaire et de favoriser l'agrégation du SCO. Avec un $\triangle E_{S T}$ nul, TADF9 possède non seulement des propriétés TADF mais aussi des propriétés d'émission induite par l'agrégation et les performances de $D 50\left(\mathrm{~V}_{\text {on }}: 2.7 \mathrm{~V}\right.$ et EQE : $\left.23.5 \%\right)$ sont tout à fait remarquables pour une émission jaune.

\subsection{Emission rouge des OLEDs TADF}

D52-D54 sont des OLED-TADF à émetteur TADF pur (TADF11-TADF13) (Figure 14, Tableau 10). TADF11-TADF12 [103] ont toutes les deux une architecture D-A avec une triphénylamine comme unité donneur d'électrons et une unité acénaphto[1,2- $b$ ]quinoxaline-8,9-dicarbonitrile (TADF11) ou acénaphto[1,2-b] pyrazine-8,9-dicarbonitrile (TADF12) comme unité accepteur d'électrons, ces deux unités planes sont des accepteurs d'électron forts. A l'état solide, TADF11 et TADF12 forment des agrégats de type J. En effet pour les deux SCO, les groupements nitriles et les atomes d'azote des unités quinoxaline (TADF11) ou pyrazine (TADF12) sont chargés négativement alors que le reste du SCO est neutre ou chargé positivement. Cette répartition des charges entraîne à l'état solide un empilement moléculaire dans lequel les parties électronégatives d'une molécule se retrouvent proches des parties électropositives d'une seconde molécule. Ces interactions entrainent une modulation des propriétés optiques de ces SCOs avec des émissions jaunes ( $\lambda_{\text {fluo }}: 560 \mathrm{~nm}$ pour TADF11) ou rouge-orangé ( $\lambda_{\text {fluo }}: 609 \mathrm{~nm}$ pour TADF12) en solution diluée qui se décalent fortement vers le rouge à l'état solide ( $\lambda_{\text {fluo }}: 716 \mathrm{~nm}$ pour TADF11 \& $\lambda_{\text {fluo }}: 777 \mathrm{~nm}$ pour TADF12). Ainsi, D52 et D53 émettent dans le proche infrarouge avec des EQE de $5.1 \%$ (TADF12, $765 \mathrm{~nm}$ ) et $3.5 \%$ (TADF11, $711 \mathrm{~nm})$. 

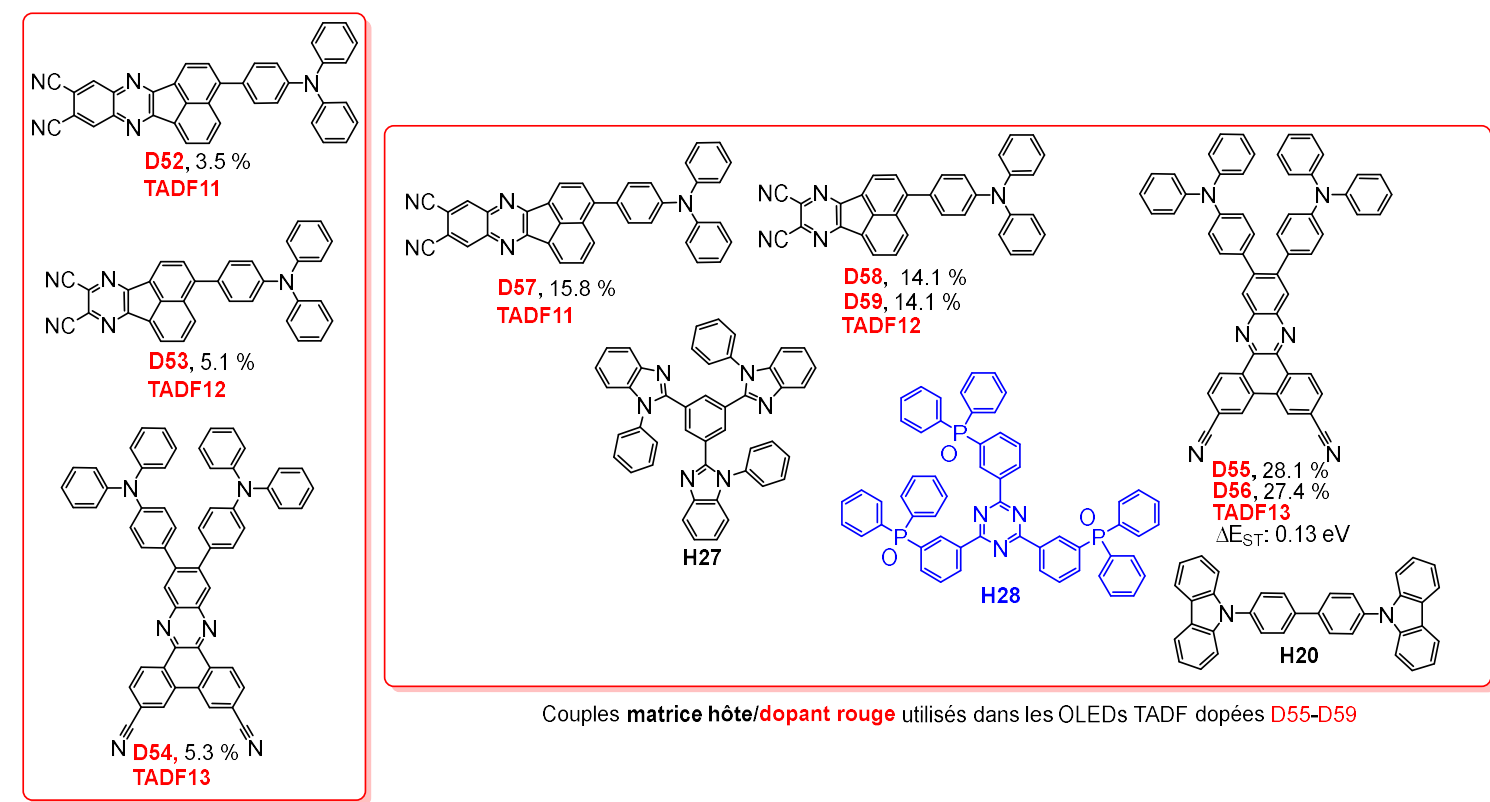

Couples matrice hôte/dopant rouge utilisés dans les OLEDs TADF dopées D55-D59

OLEDs TADF non dopées D52-D54

Figure 14. Exemples de SCOs à propriété TADF pour une émission rouge

\begin{tabular}{|c|c|c|c|c|c|c|c|c|}
\hline & Architecture du dispositif & $\begin{array}{l}\mathrm{V}_{\text {on }} \\
\text { (V) }\end{array}$ & $\begin{array}{l}\mathrm{EQE}_{\max } \\
(\%)\end{array}$ & $\begin{array}{l}\mathrm{CE}_{\max } \\
(\mathrm{cd} / \mathrm{A})\end{array}$ & $\begin{array}{c}P E_{\max } \\
(\mathrm{Im} / \mathrm{W})\end{array}$ & $\begin{array}{c}\lambda_{\max } \\
(\mathrm{nm})\end{array}$ & $\begin{array}{c}\text { CIE } \\
(x, y)\end{array}$ & Réf. \\
\hline \multicolumn{9}{|c|}{ Dispositif TADF-OLED multicouche Rouge } \\
\hline D52 & $\begin{array}{l}\text { ITO/HAT-CN(5nm)/NPB(70nm)/mCP(5nm)/TADF11 } \\
(30 \mathrm{~nm}) / \mathrm{H} 27(40 \mathrm{~nm}) / \mathrm{LiF}(1 \mathrm{~nm}) / \mathrm{Al}\end{array}$ & 2.5 & 3.5 & - & - & 711 & - & [103] \\
\hline D53 & $\begin{array}{l}\text { ITO/HAT-CN }(5 \mathrm{~nm}) / \mathrm{NPB}(70 \mathrm{~nm}) / \mathrm{mCP}(5 \mathrm{~nm}) / \mathrm{TADF} 12 \\
10 \%(30 \mathrm{~nm}) / \mathrm{H} 27(40 \mathrm{~nm}) / \mathrm{LiF}(1 \mathrm{~nm}) / \mathrm{Al}(150 \mathrm{~nm})\end{array}$ & 2.5 & 5.1 & - & - & 765 & Proche IR & [103] \\
\hline D54 & $\begin{array}{l}\text { ITO/HAT-CN(10nm)/TAPC(40nm)/TCTA(10nm) /TADF13 } \\
(20 \mathrm{~nm}) / \mathrm{TmPyPB}(55 \mathrm{~nm}) / \mathrm{Liq}(2 \mathrm{~nm}) / \mathrm{Al}(120 \mathrm{~nm})\end{array}$ & 3.1 & 5.3 & 1.4 & 1.3 & 680 & $\begin{array}{l}\text { Proche IR } \\
0.69,0.30 \\
\end{array}$ & [104] \\
\hline D55 & $\begin{array}{l}\text { ITO/HAT-CN(10nm)/TAPC(40nm)/TCTA(10nm) /H20: } \\
\text { H28(1:1):TADF13 15\%(20nm)/TmPyPB(55nm)/Liq } \\
(2 \mathrm{~nm}) / \mathrm{Al}(120 \mathrm{~nm})\end{array}$ & 2.4 & 28.1 & 20.0 & 26.3 & 648 & $\begin{array}{c}\text { Rouge } \\
\text { profond } \\
0.66,0.34 \\
\end{array}$ & [104] \\
\hline D56 & $\begin{array}{l}\text { ITO/HAT-CN(10nm)/TAPC(40nm)/TCTA(10nm)/H20: } \\
\text { TADF13 15\% (20nm)/TmPyPB (55nm)/Liq(2nm)/AI } \\
(120 \mathrm{~nm})\end{array}$ & 3.0 & 27.4 & 30.0 & 31.6 & 628 & $0.65,0.35$ & [104] \\
\hline D57 & $\begin{array}{l}\text { ITO/HAT-CN(5nm)/NPB(70nm)/mCP(5nm)/H27: } \\
\text { TADF11 5\% (30nm)/H27(40nm)/LiF(1nm)/Al(150nm) }\end{array}$ & 3.3 & 15.8 & - & - & 630 & $\begin{array}{l}\text { Rouge } \\
\text { profond }\end{array}$ & [103] \\
\hline D58 & $\begin{array}{l}\text { ITO/HAT-CN(5nm)/NPB(70nm)/mCP(5nm)/H27 : } \\
\text { TADF12 10\% (30nm)/H27(40nm)/LiF(1nm)/Al(150nm) }\end{array}$ & 4.3 & 14.1 & - & - & 657 & $\begin{array}{l}\text { Rouge } \\
\text { profond }\end{array}$ & [103] \\
\hline D59 & $\begin{array}{l}\text { ITO/HAT-CN(5nm)/NPB(70nm)/TCTA(5nm)/H27: } \\
\text { TADF12 15\% (30nm)/H27(40nm)/LiF(1nm)/Al(150nm) }\end{array}$ & 2.5 & 14.1 & - & - & 700 & Proche IR & [103] \\
\hline
\end{tabular}

Tableau 10. Exemples de performances de dispositifs OLED TADF à émission rouge

Les propriétés TADF de TADF11 et TADF12 ne sont pas observées en solution diluées ni à l'état solide lorsque le SCO est en faible concentration, 0.1 \%, dans une matrice hôte. Elles n'apparaissent qu'en concentration plus élevée, 1-10\%, du SCO dans la matrice de H27. Ces observations sont en accord avec l'existence d'interactions moléculaires à partir d'une certaine concentration et la formation d'agrégat de type J qui entrainent la diminution de l'écart énergétique entre les états excités singulet et triplet $\left(\Delta \mathrm{E}_{\mathrm{ST}}: 0.3 \mathrm{eV}\right)$ et induisent une émission de fluorescence activée thermiquement. D57-D59 utilisant comme EML TADF11 ou TADF12 dopés dans H27 sont donc plus efficace que D52 et D53 et atteignent des EQE de $15.8 \%$ dans le rouge (D57) et de $14.1 \%$ dans le proche infra-rouge (D59).

TADF13 est un SCO TADF à émission rouge constitué de deux unités donneur d'électrons triphénylamine et d'un accepteur d'électrons plan dibenzo[a,c]phénazine-3,6-dicarbonitrile. [104] Son $\Delta \mathrm{E}_{\text {ST }}$ est de $0.13 \mathrm{eV}$. Utilisé dans une OLED-TADF non dopée, D54, la diode émet dans le proche infrarouge $(680 \mathrm{~nm},(0.69,0.30))$ avec un rendement dépassant $5 \%$ le classant dans les meilleures OLEDTADF non dopées émettant dans cette gamme de longueur d'ondes.

Les performances de cet émetteur TADF sont améliorées lorsque TADF13 est utilisé comme dopant de H20 avec un EQE atteignant $27.4 \%$ et une émission décalée vers le rouge (D56, $628 \mathrm{~nm}$ ).[104] Enfin, utilisé comme dopant d'une " matrice mixte " $\mathbf{H 2 0 : H 2 8}$, le rendement de la diode D55 dépasse $\mathbf{2 8} \%$ $(648 \mathrm{~nm})$ s'inscrivant comme la diode TADF dopée rouge la plus efficace de la littérature. Qu'est-ce 
qu'une matrice mixte ? C'est l'appellation donnée à l'utilisation de deux matrices dans l'EML d'une OLED. Quel est l'intérêt d'utiliser une matrice mixte ? Individuellement, $\mathbf{H 2 0}$ donneur d'électron et $\mathbf{H 2 8}$ (1,3,5-triazine-2,4,6-triyl)tris(benzène-3,1-diyl)tris(diphenylphosphine oxyde) accepteur d'électron émettent à la même longueur d'onde (396 et $395 \mathrm{~nm}$ respectivement) alors qu'un mélange de $\mathbf{H 2 0}$ et de $\mathbf{H 2 8}$ émet à $480 \mathrm{~nm}$ indiquant un décalage d'émission vers le rouge. Ce décalage indique la formation d'un exciplex (complexe intermoléculaire à l'état excité) du fait d'interactions DonneurAccepteur intermoléculaire entre les deux matrices. La matrice mixte a ainsi plusieurs mérites : elle diminue la barrière d'injection des charges en utilisant des molécules pauvre et riche en électrons, elle supprime la désactivation des excitons en diluant leur concentration, elle favorise l'efficacité du transfert énergétique de la matrice hôte vers le dopant et elle induit un environnement polaire pour le dopant.

\subsection{Emission blanche des OLEDs TADF}

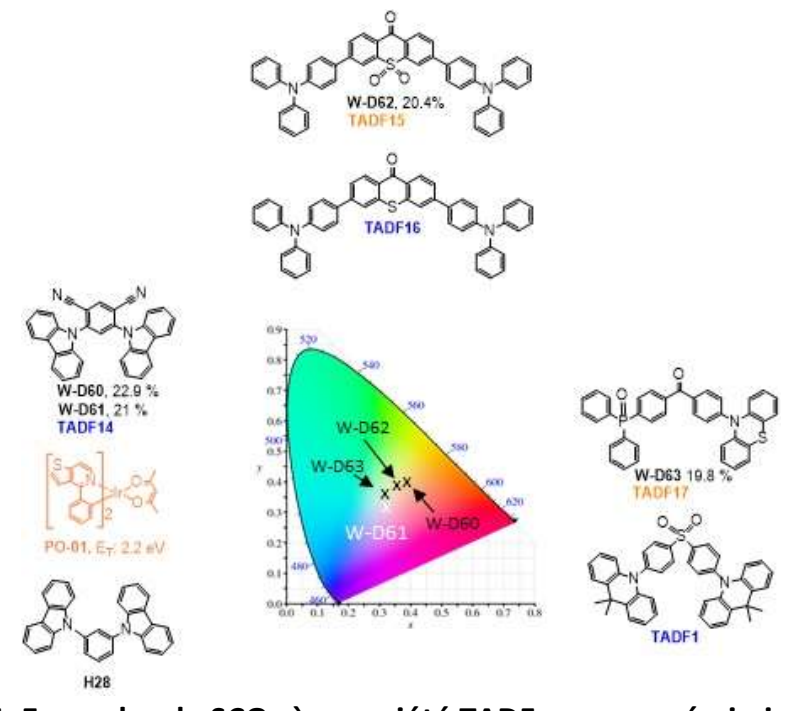

Figure 15. Exemples de SCOs à propriété TADF pour une émission blanche

Il existe plusieurs types de diodes à émission blanches basées sur des SCOs à propriétés TADF (Figure 15, Tableau 11). W-D60 et W-D61 [105] sont des « OLEDs blanches hybrides » dont une couche de SCO à propriétés TADF, TADF14, dopée par le dopant phosphorescent jaune-orange PO-01 est entourée de deux couches émissives bleues (H28 dopé par TADF14). En effet, TADF14 possède à la fois des propriétés d'émission bleue TADF[105] mais aussi un $E_{T}(2.72 \mathrm{eV})$ adapté au dopant phosphorescent jaune $\mathrm{PO}-01$ utilisé. Les auteurs de ces travaux décrivent une OLED ayant comme EML le couple TADF14 dopée au PO-01 qui émet une lumière jaune avec un EQE $E_{\max }$ de $24.9 \%$.[105] Dans W-D60 et W-D61, sont combinées les émissions bleues et jaunes de ces diodes conduisant à une émission de lumière blanche de coordonnées chromatiques variables selon l'épaisseur de la couche émissive jaune TADF14:PO-01. W-D61 atteint un EQE $E_{\max }$ de $21 \%$, émet un blanc froid (0.31, 0.33 ) alors que $W-D 60$ dont la couche émissive jaune est plus épaisse atteint $22.9 \%$ d’EQE et émet un blanc plus chaud $(0.39,0.43)$.

\begin{tabular}{|c|c|c|c|c|c|c|c|c|}
\hline & Architecture du dispositif & $\begin{array}{l}V_{\text {on }} \\
\text { (V) }\end{array}$ & $\begin{array}{l}E_{\text {EQEmax }} \\
(\%)\end{array}$ & $\begin{array}{l}\mathrm{CE}_{\max } \\
(\mathrm{cd} / \mathrm{A})\end{array}$ & $\begin{array}{l}P E_{\max } \\
(\mathrm{Im} / \mathrm{W})\end{array}$ & $\begin{array}{l}\lambda_{\max } \\
(\mathrm{nm})\end{array}$ & $\mathrm{ClE}$ & Réf. \\
\hline W-D60 & $\begin{array}{l}\text { ITO(150nm)/PEDOT:PSS }(60 \mathrm{~nm}) / \mathrm{TAPC}(20 \mathrm{~nm}) / \\
\mathrm{mCP}(10 \mathrm{~nm}) / \mathrm{H} 28: \mathrm{TADF} 14 \text { 15\%(13.5nm)/ } \\
\text { TADF14:PO-01 5\% (3nm)/H28:TADF14(13.5nm) } \\
\text { /TSPO1 }(35 \mathrm{~nm}) / \mathrm{LiF}(1 \mathrm{~nm}) / \mathrm{Al}(200 \mathrm{~nm})\end{array}$ & 6 & 22.9 & - & - & $\begin{array}{c}462,558, \\
585(\text { sh) }\end{array}$ & $\begin{array}{l}0.39 \\
0.43\end{array}$ & [105] \\
\hline W-D61 & $\begin{array}{l}\text { ITO(150nm)/PEDOT:PSS }(60 \mathrm{~nm}) / \mathrm{TAPC}(20 \mathrm{~nm}) / \\
\mathrm{mCP}(10 \mathrm{~nm}) / \mathrm{H} 28: T A D F 14 \text { 15\%(4.25nm)/ } \\
\text { TADF14:PO-01 5\% (1.5nm)/H28:TADF14 } \\
(4.25 \mathrm{~nm}) / \mathrm{TSPO} 1(35 \mathrm{~nm}) / \mathrm{LiF}(1 \mathrm{~nm}) / \mathrm{Al}(200 \mathrm{~nm})\end{array}$ & 6 & 21 & - & - & $\begin{array}{c}462,558, \\
585(\text { sh) }\end{array}$ & $\begin{array}{l}0.31 \\
0.33\end{array}$ & [105] \\
\hline W-D62 & $\begin{array}{l}\mathrm{ITO}(95 \mathrm{~nm}) / \mathrm{TAPC}(25 \mathrm{~nm}) / \mathrm{H} 20: T A D F 15 \text { 6\%(30nm) } \\
/ \text { H20(3nm)/H20:TADF16 3\%(2nm)/TmPyPB } \\
(55 \mathrm{~nm}) / \mathrm{LiF}(1 \mathrm{~nm}) / \mathrm{Al}(100 \mathrm{~nm})\end{array}$ & 3.4 & 20.4 & 49.5 & 48.6 & 478,577 & $\begin{array}{l}0.34 \\
0.41\end{array}$ & [106] \\
\hline W-D63 & $\begin{array}{l}\text { ITO/PEDOT:PSS(40nm)/H20(20nm)/TADF17 } \\
(4 \mathrm{~nm}) / \text { TADF1 }(16 \mathrm{~nm}) / \mathrm{H} 27(40 \mathrm{~nm}) / \mathrm{Mg}: \mathrm{Ag}\end{array}$ & 5 & 19.8 & 41.4 & 21.7 & 486,566 & $\begin{array}{l}0.30 \\
0.38\end{array}$ & [107] \\
\hline
\end{tabular}

Tableau 11. Exemples de performances de dispositifs OLED TADF à émission blanche 
W-D62 [106] comporte deux couches émissives à base de deux émetteurs à propriétés TADF de couleurs complémentaires bleues (TADF15) et jaunes (TADF16) indépendantes. La matrice hôte $\mathbf{H 2 0}$ des émetteurs fluorescents est utilisée pour séparer les deux zones d'émission. W-D62 émet dès $3.4 \mathrm{~V}$ une lumière blanche de coordonnées chromatiques $(0.34,0.41)$ et montre un $\mathrm{EQE}_{\max }$ dépassant $20 \%$.

W-D63 [107] comporte une couche émissive double composée de deux SCOs à propriétés TADF émetteur de lumière bleue (TADF1) ou de lumière jaune (TADF17) utilisés comme émetteurs TADF pur. Dans W-D63, les deux couches émissives à base de TADF1 et TADF17 sont positionnées l'une à côté de l'autre dans le centre du dispositif. Le spectre d'EL de W-D63 montre l'émission bleue centrée à $480 \mathrm{~nm}$ et l'émission jaune centrée à $580 \mathrm{~nm}$ produisant une émission globalement blanche. Un travail de design architectural de la diode en fonction de l'épaisseur relative des deux couches émissives TADF a permis d'atteindre un $\mathrm{EQE}_{\max }$ de $19.8 \%$ et une lumière blanche de coordonnées chromatiques $(0.30,0.38)$.

Comparées aux performances des PhOLEDs à émission blanche (W-D36-W-D40), les performances de W-D60-D63 sont un peu moins élevées tant au niveau de la tension de fonctionnement des diodes ( $V_{\text {on }}$ plus élevé en moyenne) que de la valeur des EQE $E_{\max }$ atteints (22.9-19.8 \% pour W-D60-D63 et 29$26.4 \%$ pour W-D36-W-D40). En revanche concernant la « qualité » de la couleur blanche émise, WD61 correspond à un blanc quasiment pur (CIE : $0.31,0.33$ vs $\mathrm{CIE}: 0.33,0.33$ pour le blanc pur) alors que les coordonnées chromatiques des autres blancs se déplacent vers le jaune (W-D60 et W-D62) voire vers le vert (W-D63).

En conclusion, les OLEDs-TADF ont connu un développement fantastique depuis 2009. Aujourd'hui, des OLED-TADF non dopées ont conduit à l'émission de lumière bleue ou verte (D41 \& D45) dans des dispositifs dont les EQE atteignent $20 \%$. Intégrés dans des SCOs hôtes, les EQE des OLEDs TADF dopées émettant de la lumière bleue ou verte dépassent aujourd'hui $35 \%[89,108]$ et atteignent presque $30 \%$ pour l'émission de lumière jaune-orange et rouge [109]. De nombreux SCOs TADF sont aujourd'hui reportés dans la littérature mais il est important de noter que le choix de la matrice hôte reste aussi important que celui du SCO TADF. Ce choix est basé sur les critères requis pour les matrices hôtes des PhOLEDs présentés page 9 , en adaptant les niveaux d'énergie de la matrice hôte à celui du SCO TADF afin d'assurer un bon transfert énergétique de la matrice hôte vers le dopant TADF ( $T_{1}$ dopant $>S_{1}$ SCO TADF). La troisième génération d'OLED-TADF est aujourd'hui en plein essor et les sociétés Cynora[110] en Allemagne, Idemitsu Kosan Co.,Ltd.[111] en relation avec la société Toray Industries, Inc.[112] ou Kyulux[113] au Japon développent régulièrement de nouvelles matrices, de nouveaux SCO TADF et les incorporent dans de nouveaux dispositifs de plus en plus performants. Cette force de frappe industrielle s'ajoute à celle des laboratoires académiques et constitue une communauté scientifique très active.

\begin{tabular}{|l|}
\hline \multicolumn{1}{|c|}{ À retenir } \\
\hline -
\end{tabular}

\section{Les OLEDs hyperfluorescentes}

Plus récemment en 2015, une quatrième génération d'OLEDs nommées « hyperfluorescent OLEDs » ou « TADF-assisted fluorescent OLED » a vu le jour.[114] L'hyperfluorescence ${ }^{T M}$ est un concept qui combine la haute pureté de couleur d'une molécule fluorescente (spectre de fluorescence fin) et la 
haute efficacité d'une molécule TADF. La couche émissive de l'OLED hyperfluorescente consiste en une matrice SCO hôte, un SCO TADF et un SCO fluorescent. Ces nouveaux dispositifs sont annoncés comme hautement efficaces, de faible coût et d'émission de couleur de qualité supérieure.

\subsection{Principe de fonctionnement d'une OLED hyperfluorescente}

Dans une OLED hyperfluorescente, la couche active est un système hôte/dopant TADF, le SCO TADF étant lui-même dopé en très faible quantité par un SCO fluorescent qui sera le seul émetteur.

Comme indiqué plus haut pour les OLEDs TADF dopées, lors de la mise sous tension du dispositif, des électrons et des trous provenant des électrodes sont injectés dans le SCO TADF via la matrice hôte, ils se combinent pour former des excitons dans un rapport de 25/75 singulets/triplets (Figure 16). Les excitons triplets se concentrent en excitons singulets dans le SCO TADF par RISC de $T_{1}$ vers $S_{1}$. II y a alors un transfert rapide des excitons singulets du SCO TADF vers le SCO fluorescent ( $\mathrm{S}_{1}$ vers $\mathrm{S}_{1}$ ) et émission de lumière à partir de ce dernier.

Au bilan, tous les excitons formés dans la matrice hôte se retrouvent à l'état d'excitons singulets dans le SCO TADF, sont alors rapidement transférés dans le SCO fluorescent et reviennent à l'état fondamental en émettant de la lumière par fluorescence (retour de $S_{1}$ vers $S_{0}$ ). Ce transfert rapide conduit à un IQE pouvant atteindre $100 \%$, à une lumière émise de haute pureté (un seul émetteur fluorescent et un spectre d'EL fin) et à une durée de vie de la diode plus élevée que celle de l'OLEDTADF (stabilité de la molécule fluorescente).[115]

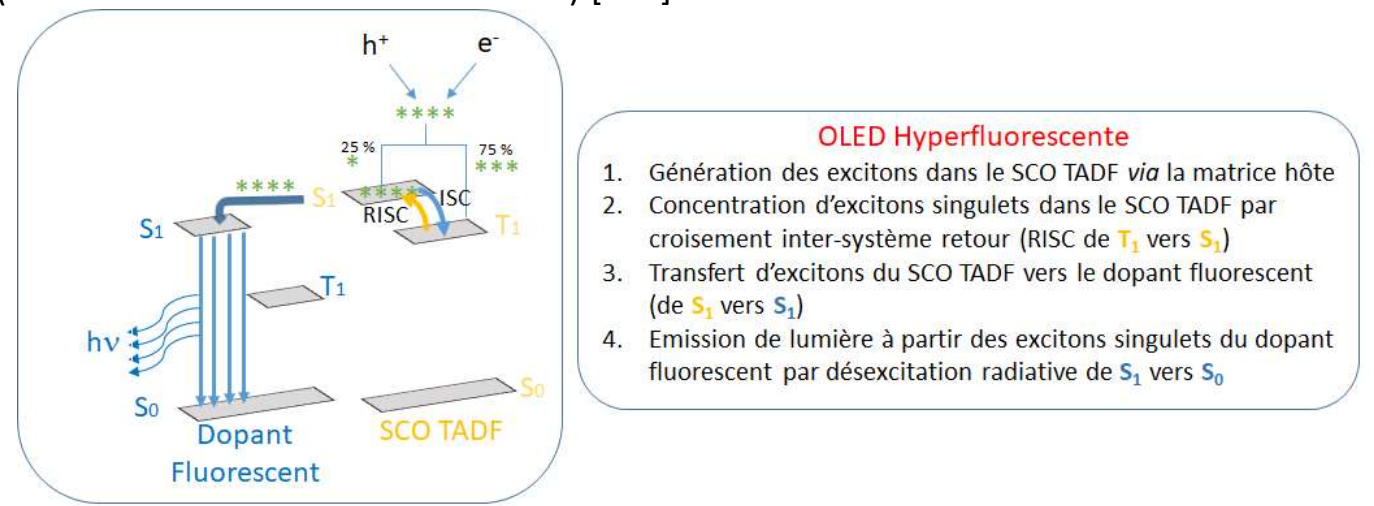

Figure 16. Principe de fonctionnement d'une OLED hyperfluorescente

Les propriétés requises pour qu'un SCO puisse être utilisé dans une couche active d'une OLED hyperfluorescente sont similaires à celles décrites pour le SCO d'une OLED fluorescente pour les items (i)-(iv) (décrits pages 4/5) avec également la contrainte concernant l'écart entre les niveaux d'énergie $S_{1}$ et $T_{1}\left(\triangle E_{S T}\right)$ du SCO TADF (décrite page 18) et une contrainte supplémentaire concernant le niveau $\mathrm{S}_{1}$ du SCO fluorescent qui doit être inférieur au niveau $\mathrm{S}_{1}$ du SCO TADF.

\subsection{Exemples d'OLEDs hyperfluorescentes}

Les exemples présentés Figure 17 sont issus principalement de la première communication en 2014 du groupe d'Adachi[25] sur les diodes hyperfluorescentes et permettent de visualiser comment, en choisissant différentes combinaisons matrice hôte/dopant TADF/dopant fluorescent, on peut obtenir une émission dans diverses gammes de longueurs d'onde par fluorescence dans des dispositifs présentant des performances intéressantes.

Dans ces exemples, la matrice hôte joue le même rôle que dans les OLEDs TADF dopées, elle permet de diluer le dopant TADF dans la matrice et d'augmenter l'efficacité des propriétés TADF du SCO en diminuant les phénomènes de désactivation des excitons triplets du SCO TADF (désactivation par effet de concentration, l'annihilation triplet-triplet, la migration des excitons,...[91] voir page 18). 


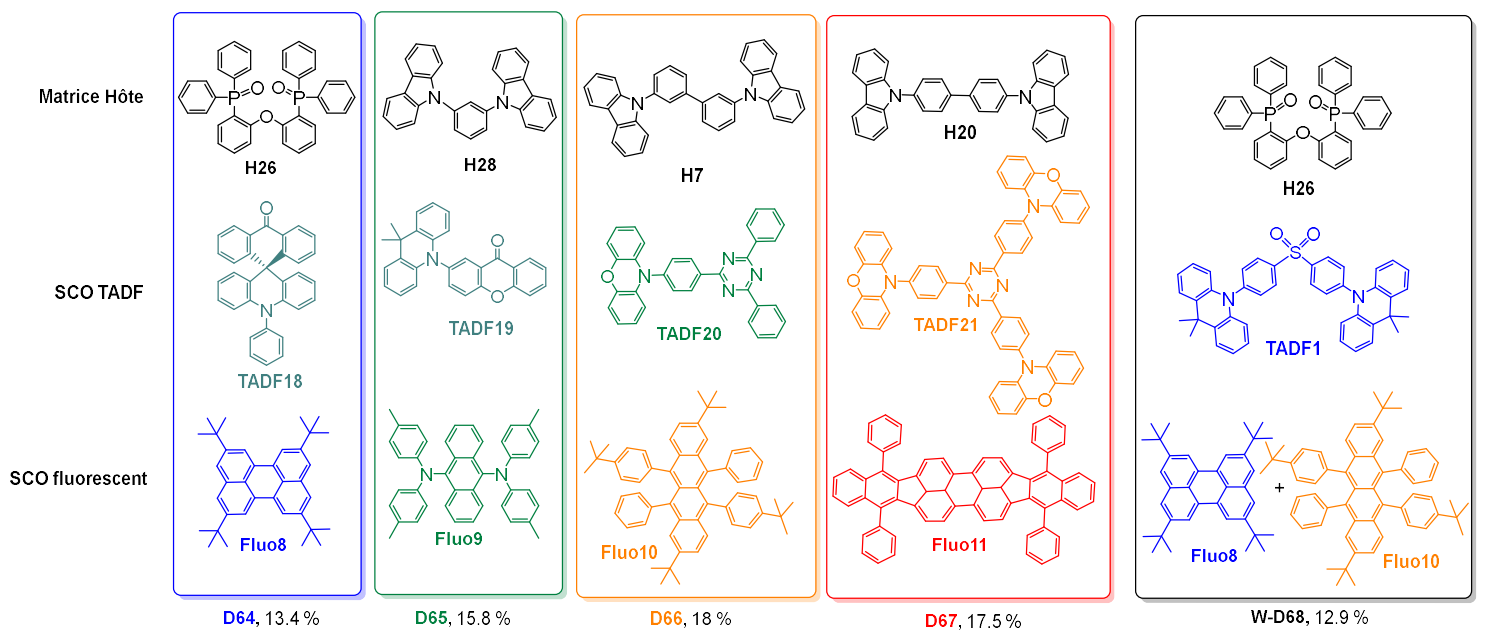

Figure 17. Exemples de SCOs fluorescent utilisés en OLEDs hyperfluorescentes bleu, verte, jaune-orange, rouge ou blanches

\begin{tabular}{|c|c|c|c|c|c|c|c|c|}
\hline & Architecture du dispositif & $\begin{array}{l}V_{\text {on }} \\
\text { (V) }\end{array}$ & $\begin{array}{l}E Q E_{\max } \\
(\%)\end{array}$ & $\begin{array}{l}C E_{\max } \\
(\mathrm{cd} / \mathrm{A})\end{array}$ & $\begin{array}{l}\mathrm{PE} \mathrm{Emax}_{\mathrm{max}} \\
(\mathrm{Im} / \mathrm{W})\end{array}$ & $\begin{array}{c}\lambda_{\max } \\
(\mathrm{nm})\end{array}$ & CIE & Réf. \\
\hline D64 & 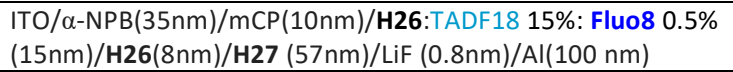 & 4.7 & 13.4 & 27 & 18 & 462 & $\begin{array}{l}0.17 \\
0.30\end{array}$ & [25] \\
\hline D65 & $\begin{array}{l}\text { ITO/TAPC(35nm)/H28:TADF19 50\%:Fluo9 1\%(15nm)/H27 } \\
(65 \mathrm{~nm}) / \mathrm{Li}(0.8 \mathrm{n}) / \mathrm{Al}(100 \mathrm{~nm})\end{array}$ & 3.0 & 15.8 & 45 & 47 & 525 & $\begin{array}{l}0.29 \\
0.59\end{array}$ & [25] \\
\hline D66 & $\begin{array}{l}\text { ITO/TAPC(35nm)/H7:TADF20 25\%:Fluo10 1\%(30nm)/T2T } \\
(10 \mathrm{~nm}) / \mathrm{Alq}_{3}(55 \mathrm{~nm}) / \mathrm{LiF}(0.8 \mathrm{~nm}) / \mathrm{Al}(100 \mathrm{~nm})\end{array}$ & 3.2 & 18.0 & 60 & 58 & 558 & $\begin{array}{l}0.45 \\
0.53\end{array}$ & [25] \\
\hline D67 & $\begin{array}{l}\text { ITO/TAPC(35nm)/H20:TADF21 15\%:Fluo11 1\%(30nm)/H27 } \\
(65 \mathrm{~nm}) / \mathrm{LiF}(0.8 \mathrm{~nm}) / \mathrm{Al}(100 \mathrm{~nm})\end{array}$ & 3.0 & 17.5 & 25 & 28 & 610 & $\begin{array}{l}0.61 \\
0.39\end{array}$ & [25] \\
\hline W-D68 & $\begin{array}{l}\text { ITO/PEDOT:PSS(60nm)/TAPC(20nm)/mCP(10nm)/H26:TADF1 } \\
\text { 50\%:Fluo8 1\%:Fluo10: 0.5\%(25nm)/TSPO1(5nm)/H27(30nm) } \\
\text { /LiF(1nm)/Al(200nm) }\end{array}$ & 2.5 & 12.9 & 29.8 & 31.1 & & $\begin{array}{l}0.26, \\
0.32\end{array}$ & [45] \\
\hline
\end{tabular}

Tableau 12. Exemples de performances de dispositifs OLED hyperfluorescente

Dans D64, la couche émissive est constituée d'une matrice hôte H26, d'un dopant TADF18 (phénylspiro[acridine-anthracénone]) émetteur de couleur cyan à l'état solide (500 nm) et d'un dopant fluorescent Fluo8 présent en faible quantité (0.5\%).[25] D63 émet une lumière bleue centrée à 462 $\mathrm{nm}$ et atteint un EQE $E_{\max }$ supérieur à $13 \%$ (Tableau 12). La couleur émise par la diode provient uniquement du dopant fluorescent Fluo8 sans aucune participation de l'émission de TADF18 pourtant présent en quantité importante (15\%). Cela met en évidence l'efficacité des transferts d'énergie au sein de ce système. Il est intéressant de noter qu'une diode de même architecture ne contenant pas TADF18 dans l'EML émet la même lumière bleue provenant de Fluo8 mais avec un EQE n'atteignant pas $1.5 \%$.[25] Le facteur 10 entre les deux EQE provient d'un transfert de la totalité des excitons (singulets et triplets via le processus RISC dans TADF18) du niveau $S_{1}$ du TADF18 vers le niveau $S_{1}$ de Fluo8. C'est une preuve de l'efficacité du concept d'hyperfluorescence.

Dans D65, la couche émissive est une matrice de $\mathbf{H 2 8}$ dopée à $50 \%$ par TADF19 et à $1 \%$ par le fluorescent vert Flu09. [25] Cette diode émet dès $3 \mathrm{~V}$ une lumière verte provenant uniquement de Fluo9 et atteint un EQE de $15.8 \%$.

D66 et D67 utilisant aussi la fluorescence des dopants jaune-orange Fluo10[25] ou rouge Fluo11[25] sont particulièrement efficaces. On notera dans D66, la présence entre l'EML et l'EIL $\left(\mathrm{Alq}_{3}\right) \mathrm{d}^{\prime}$ une couche de 2,4,6-tris(biphenyl-3-yl)-1,3,5-triazine (T2T) utilisé par les auteurs comme transporteur d'électrons du fait de sa mobilité d'électron élevée $\left(\mu_{\mathrm{e}}=c a .1 .2 \times 10^{-4} \mathrm{~cm}^{2} \mathrm{~V}^{-1} \mathrm{~s}^{-1}\right)$ et comme bloqueur d'excitons du fait de son $E_{T}$ élevée (2.8 eV).[116]

Le dernier exemple de diode hyperfuorescente a son EML constituée d'une matrice $\mathbf{H} \mathbf{2 6}$ dopée à $50 \%$ par TADF1 (SCO émetteur de bleu, déjà utilisé dans D41 et W-D62) mais aussi par deux dopants fluorescents respectivement bleu (Fluo8) et orange (Fluo10).[45] En jouant sur le taux de dopage de ces deux dopants fluorescents, les auteurs ont pu obtenir avec la diode W-D68, une émission de lumière blanche dès $2.5 \mathrm{~V}$ et atteindre un $\mathrm{EQE}_{\max }$ de $12.9 \%$. 


\section{Conclusion}

Les exemples présentés ci-dessus ont permis de montrer l'intérêt de la nouvelle génération de diodes hyperfluorescentes. Ces nouveaux dispositifs seront sans doute au cœur des OLEDs dans les années à venir car de nombreux groupes travaillent actuellement au développement de ces nouvelles OLEDs. Les sociétés Cynora[110] en Allemagne et Kyulux[113] au Japon présentent régulièrement de nouveaux résultats en ce sens et insistent sur le grand avantage de la qualité de la couleur émise par le SCO fluorescent et sur l'augmentation du rendement d'émission et de la durée de vie de ces nouvelles diodes. Un projet de 4 millions d'euros financé par le programme de recherche et d'innovation Horizon 2020 de l'UE est coordonné par Merck, en étroite collaboration avec quatre autres partenaires européens: Microoled (France), Fraunhofer-IOF (Allemagne), Durham University (Royaume-Uni) et Intelligentsia Consultants (Luxembourg). Durant trois ans, ce projet HyperOLED[117] se concentrera sur le développement d'OLED à haute performance en combinant des hôtes moléculaires à fluorescence retardée activée thermiquement (TADF) avec de nouveaux émetteurs de fluorescence spécifiquement adaptés. Dans le cadre de ce projet, les partenaires s'attendent à contribuer également au développement d'appareils électroniques minces, organiques et de grande surface, une technologie émergente à fort potentiel de croissance.

À retenir

- Dans une OLED Hyperfluorescente:

- La couche active est un trio matrice hôte/dopant TADF/SCO fluorescent

- I'IQE peut atteindre $100 \%$ si le $\triangle E_{s T}$ du dopant TADF est faible et le phénomène RISC total dans le dopant TADF et enfin si tous les excitons singulet du dopant TADF sont transférés dans le SCO fluorescent.

- la couleur de la lumière émise est celle du SCO fluorescent

\section{Conclusion générale}

Les différentes générations de diodes électroluminescentes organiques présentées ci-dessus montrent l'évolution de ces nouvelles techniques pour l'affichage et l'éclairage et ont permis de mettre en exergue les différents modes de fonctionnement et les transferts d'énergie dans ces dispositifs. L'ensemble des exemples présentés dans cette revue, même s'ils ne sont pas tous au niveau de l'état de l'art, permettent de montrer l'intérêt que l'on a à trouver, pour chaque type de diode (OLED, PhOLED, TADF-OLED ou OLED-Hyperfluorescente), le ou les SCO composant le dispositif dont les propriétés sont les mieux adaptées afin d'atteindre un dispositif hautement performant.

L'avenir des diodes électroluminescentes organiques reposera sur leurs faibles coûts de fabrication, leurs faibles consommations électriques, leurs durées de vie en fonctionnement et leurs faibles coûts de recyclage (diminution de l'empreinte écologique) tout autant que sur leurs efficacités. Les chimistes, les physiciens et les électroniciens travaillent ensemble à fabriquer des dispositifs simples à mettre en œuvre, adaptables à des supports souples, efficaces, à longue durée de vie et facilement recyclables après leur utilisation. Il reste encore du travail, mais les résultats de plus en plus encourageants et l'utilisation des premiers dispositifs dans différents appareils électroniques commerciaux est la preuve de l'intérêt croissant porté à ces nouveaux dispositifs électroniques.

\section{Remerciements:}

Cyril Poriel et Joëlle Rault-Berthelot souhaitent remercier l'Université de Rennes 1, le CNRS, l'Agence Nationale de la Recherche (Projets de Recherche HOME-OLED ANR-11-BS07-0020, MenInBlue ANR14-CE05-0024 \& SpiroQuest ANR-19-CE05-0024), l'ADEME et la Région Bretagne pour leurs soutiens financiers. Les auteurs souhaitent également remercier l'ensemble de leurs collaborateurs et tout particulièrement les doctorants pour la synthèse et l'analyse physico-chimique des molécules issues de leur groupe présentées dans cet article. Nos remerciements vont aussi à B. Geffroy, au Dr. Denis Tondelier et leurs collaborateurs qui ont fabriqué et testé les PhOLEDs utilisant ces molécules comme matériaux hôtes. Les résultats des diodes D10, D23 et D31 sont le fruit d'une collaboration de notre 
groupe avec le groupe du Professeur Zuo-Quan Jiang (FUNSOM, Soochow University à Suzhou) en Chine qui est vivement remercié. 
OLED (Hyper)fluorescente ; (Hyper)fluorescent OLED

Diode électroluminescente organique dont la couche active est un (trio matrice hôte/dopant TADF/SCO fluorescent) SCO fluorescent

\title{
OLED Phosphorescente ; Phosphorescent OLED
}

Diode électroluminescente organique dont la couche active est un couple matrice hôte/dopant phosphorescent et dont l'émission provient du dopant phosphorescent.

\section{TADF-OLED ; OLED TADF}

Diode électroluminescente organique dont la couche active est un SCO émettant par fluorescence rapide et retardée activée thermiquement ou un couple matrice hôte/SCO fluorescent TADF.

\section{Exciton singulet-Exciton triplet ; singlet exciton-triplet exciton}

Un exciton est, en physique, une quasi-particule que l'on peut voir comme une paire électron-trou liée par des forces de Coulomb. Selon la statistique de spin il se forme $25 \%$ d'exciton singulet et $75 \%$ d'exciton triplet.

\section{OLED monocouche ; Single-Layer OLED}

Diode électroluminescente organique dont la couche active est un SCO fluorescent ou un couple matrice hôte/dopant prise en sandwich entre une anode et une cathode sans autre couche additionnelle.

\section{OLED multicouche ; Multi-Layer OLED}

Diode électroluminescente organique dont la couche active est un SCO fluorescent ou un couple matrice hôte/dopant entourée de plusieurs couches de SCO aidant à l'injection, au transport et/ou au confinement des charges afin de favoriser la recombinaison électron/trous dans le centre de la couche émissive.

\section{Sigles}

\author{
BmPyPB: 1,3-bis[3,5-di(pyridin-3-yl)-phényl]benzène, ETL, HBL \\ BPhen : 4,7-diphényl-1,10-phénanthroline, EIL, ETL et HBL \\ CE pour current efficiency: rendement de courant $(\mathrm{cd} / \mathrm{A})$ \\ CIE $(x, y)$ : Coordonnée chromatique de la Commission Internationale de l'Eclairage \\ DF pour delayed fluorescence : fluorescence retardée \\ $D-\pi-A$ : Donneur-système $\pi-A c c e p t e u r$ \\ D-Spiro-A : Donneur-Spiro-Accepteur \\ EBL pour Electron Blocking Layer : couche de blocage des électrons \\ EIL pour Electron Injecting Layer : couche d'injection d'électrons \\ EL : électroluminescene \\ EML pour emitting layer: couche émissive de la diode \\ EQE pour External Quantum Efficiency: rendement quantique externe de la diode (\%) \\ ET: état triplet \\ ETL pour Electron Transporting Layer : couche de blocage des électrons \\ fac-Ir(iprpmi) ${ }_{3}$ : fac-Tris[(2,6-diisopropylphenyl)-2-phenyl-1H -imidazo[e ]]iridium(III), émetteur phosphorescent bleu \\ (fbi) $2 \operatorname{lr}(\mathrm{acac})$ : bis(2-(9,9-diéthyl-fluorèn-2-yl)-1-phényl-1H-benzo[d]imidazolato)(acétylacétonate)iridium(III), émetteur phosphorescent \\ orange \\ Flrpic: Bis[2-(4,6-difluorophényl)pyridinato- $\left.C^{2}, N\right]$ (picolinato)iridium(III) , émetteur phosphorescent bleu \\ HAT-CN: Dipyrazino[2,3-f:2', 3'-h]quinoxaline 2,3,6,7,10,11-hexacarbonitrile, HIL \\ HBL pour Hole Blocking Layer : couche de blocage des trous \\ HF-OLED pour Hyperfluorescent-OLED : OLED hyperfluorescente \\ HIL pour Hole Injecting Layer : couche d'injection de trous \\ HOMO pour Highly Occupied Molecular Orbital : Orbitale Moléculaire Haute Occupée \\ HTL pour Hole Transporting Layer : couche de transport de trous \\ IQE pour Internal Quantum Efficiency: rendement quantique interne de la diode (\%) \\ $\operatorname{Ir}(\mathrm{cb})_{3}$ : [[5-(1,1-diméthyléthyl)-3-phényl-1H-imidazo[4,5-b]pyrazin-1-yl-2(3H)-ylidène]-1,2-phénylène]bis[[6-(1,1-diméthyléthyl)-3-phényl- \\ $1 \mathrm{H}$-imidazo[4,5-b]pyrazin-1-yl-2(3H)-ylidène]-1,2-phénylène]iridium, émetteur phosphorescent bleu \\ Ir(dbi) $)_{3}$ : fac-tris[1-(2,4-diisopropyldibenzo[b,d]furan-3-yl)-2-phényl-1H-imidazole] iridium(III), émetteur phosphorescent bleu
}


$\operatorname{Ir}(\mathrm{dmdppr} \text {-dmp })_{2}$ (divm) : émetteur phosphorescent rouge

$\operatorname{Ir}(\mathrm{MDQ})_{2}$ (acac) : Bis(2-méthyldibenzo[f,h]quinoxaline)(acétylacétonate) iridium(III), émetteur phosphorescent rouge

$\operatorname{Ir}(\text { piq })_{3}$ : Tris(1-phénylisoquinoline)iridium(III), émetteur phosphorescent rouge

$\operatorname{Ir}(\text { ppy) })_{2}$ (acac) : Bis[2-(2-pyridinyl-N)phényl-C](acétylacétonato)iridium(III), émetteur phosphorescent vert

$\operatorname{Ir}(\text { ppy })_{3}$ : Tris[2-phénylpyridinato- $\left.C^{2}, N\right]$ iridium(III) , émetteur phosphorescent vert

Ir(tptpy) ${ }_{2}(\mathrm{acac})$ : Acétylacétonato-bis(4-(4-tert-butylphényl)-thiéno[3,2-c]pyridinato-C2,N)iridium, émetteur phosphorescent orange

ITO pour Indium Tin Oxide: Oxyde d'indium et étain

Liq: Lithium 8-quinolinolate

LUMO Lowest Unoccupied Molecular Orbital : Orbitale Moléculaire Basse Vacante

mCP : 1,3-Bis(N-carbazolyl)benzene, $\mathbf{H 2 8}$ ou HTL

NPB: N'-di(1-naphtyl)-N,N'-diphénylbenzidine, HTL

OLED pour Organic Light-Emitting Diode: Diode électroluminescente organique

PE pour Power Efficiency: efficacité énergétique $(\mathrm{Im} / \mathrm{W})$

PEDOT:PSS : poly(3,4-éthylènedioxythiophène):poly(styrène sulfonate) de sodium, HIL

PF pour prompt fluorescence : fluorescence rapide

$\mathrm{PHC}$ pour Pure HydroCarbon : hydrocarbure

PhOLED pour Phosphorescent Organic Light-Emitting Diode: Diode électroluminescente organique phosphorescente

PO-01 : iridium(III) bis(4-phénylthiéno[3,2-c]pyridinato- $N, C 2$ ')acétylacétonate, émetteur phosphorescent orange

PPBI : 4-isopropyl-4'-méthyldiphényliodonium tetrakis(pentafluorophényl)borate, HIL

RISC pour Reverse InterSystem Crossing, croisement intersystème inverse entre T1 et S1 dans un SCO à fluorescence retardée activée thermiquement

SCO: semi-conducteur organique

TADF-OLED pour Thermally Activated Delayed Fluorescent OLED: OLED à fluorescence retardée activée thermiquement

TAPC: Di-[4-(N,N-di-p-tolyl-amino)-phényl]cyclohexane, HTL

TCTA: Tris(4-carbazoyl-9-ylphényl)amine, HIL, HTL, EBL

2-TNATA: 4,4',4"-Tris[2-naphthyl(phenyl)amino] triphenylamine, HIL

TmPyPB: 1,3,5-Tri[(3-pyridyl)-phen-3-yl]benzene, ETL et HBL

TPYMB : Tris(2,4,6-trimethyl-3-(pyridin-3-yl)phenyl)borane, EIL, ETL, HBL

TPBI: 2,2',2"-(1,3,5-Benzinetriyl)-tris(1-phényl-1-H-benzimidazole) : H27, ETL, HBL

TSPO1 : Diphenyl[4-(triphenylsilyl)phenyl]phosphine oxide, EIL, HBL

T2T : 2,4,6-tris(biphenyl-3-yl)-1,3,5-triazine, ETL, exciton blocking layer

$\mathrm{V}_{\mathrm{on}}$ : tension de seuil (Volt) 


\title{
OLED Fluorescente, OLED Phosphorescen- te, OLED à Fluorescence retardée activée thermiquement, OLED HyperFluorescente
}

\author{
par Joëlle RAULT-BERTHELOT
}

\author{
Directeur de recherche au CNRS \\ Université de Rennes, CNRS, ISCR-UMR CNRS 6226, F-35000 Rennes, France \\ Fonction :
}

et Cyril PORIEL

\author{
Directeur de recherche au CNRS \\ Université de Rennes, CNRS, ISCR-UMR CNRS 6226, F-35000 Rennes, France
}

[1] A. Bernanose, and P. Vouaux, "Electroluminescence organique: étude du mode d'émission,” J. Chim. Phys., vol. 50, pp. 261-263, 1953.

[2] M. Pope, H. P. Kallmann, and P. Magnante, "Electroluminescence in Organic Crystals," J. Chem. Phys., vol. 38, no. 3, pp. 2042-2043, 1963

[3] C. W. Tang, and S. A. VanSlyke, "Organic electroluminescent diodes," Appl. Phys. Lett., vol. 51, no. 12, pp. 913915, 1987.

[4] Z.-Q. Jiang, C. Poriel, and N. Leclerc, "Emerging organic electronics," Materials Chemistry Frontiers, vol. 4, no. 9, pp. 2497-2498, 2020.

[5] K. Ait KaciI Ali, "Les meilleurs TV OLED de novembre 2020," https://www.cnetfrance.fr/produits/meilleurestelevisions-4k-ultra-hd-39877273.htm, 2020.

[6] "https://www.ledauphine.com/magazine-lifestyle/2020/08/21/xiaomi-devoile-un-televiseur-oled-transparent."

[7] "Luminaire Osram," http://www.osram.fr/osram fr/actualites-et-savoir-faire/page-daccueil-oled/luminairesdesign-oled/index.jsp.

[8] "Eclairage OLED lumibled philips," http://www.lighting.philips.fr/lightcommunity/trends/oled/eclairage oled.wpd.

[9] "UIV Chem OLED Light - Share Natural Light, Leads a new generation of OLED lighting " http://www.ioledlight.com/.

[10] R. Mertens, "OLED lighting introduction and market status," https://www.oled-info.com/oled-lighting, 2020.

[11] "https://www.samsung.com/fr/smartphones/galaxy-fold/."

[12] "https://www.tomsguide.fr/samsung-devoile-un-ecran-pliant-oled-avec-la-plus-petite-courbure-au-monde/.",

[13] H. Sasabe, and J. Kido, "Development of high performance OLEDs for general lighting," J. Mater. Chem. C, vol. 1, no. 9, pp. 1699-1707, 2013.

[14] Organic Light-Emitting Diodes (OLEDs) Materials, Devices and Applications, University of Sheffield, UK: Woodhead Publishing, 2013.

[15] Y.-S. Tyan, "Organic light-emitting-diode lighting overview " J. Photon. Energy, vol. 1, no. 1, pp. 011009, 2011.

[16] L. S. Hung, and C. H. Chen, "Recent progress of molecular organic electroluminescent materials and devices," Mater. Sci. Eng., R, vol. 39, pp. 143-222, 2002.

[17] H. Sasabe, and J. Kido, "Recent progress in phosphorescent organic light-emitting devices," Eur. J. Org. Chem., pp. 7653-7663, 2013.

[18] L. Xiao, Z. Chen, B. Qu et al., "Recent Progresses on Materials for Electrophosphorescent Organic Light-Emitting Devices," Adv. Mater., vol. 23, pp. 926-952, 2011.

[19] Y. Tao, C. Yang, and J. Qin, "Organic host materials for phosphorescent organic light-emitting diodes," Chem. Soc. Rev., vol. 40, pp. 2943-2970, 2011.

[20] M. A. Baldo, D. F. O'Brien, Y. You et al., "Highly efficient phosphorescent emission from organic electroluminescent devices," Nature, vol. 395, no. 2, pp. 151-154, 1998.

[21] A. Endo, M. Ogasawara, A. Takahashi et al., "Thermally Activated Delayed Fluorescence from Sn4+-Porphyrin Complexes and Their Application to Organic Light Emitting Diodes - A Novel Mechanism for Electroluminescence," Adv. Mater., vol. 21, no. 47, pp. 4802-4806, 2009.

[22] T. Huang, W. Jiang, and L. Duan, "Recent progress in solution processable TADF materials for organic light-emitting diodes," J. Mater. Chem. C, vol. 6, no. 21, pp. 5577-5596, 2018.

[23] Z. Yang, Z. Mao, Z. Xie et al., "Recent advances in organic thermally activated delayed fluorescence materials," Chem. Soc. Rev., vol. 46, no. 3, pp. 915-1016, 2017.

[24] D. Volz, "Review of organic light-emitting diodes with thermally activated delayed fluorescence emitters for energyefficient sustainable light sources and displays," J. Photon. Energy, vol. 6, no. 2, pp. 020901-020901, 2016.

[25] H. Nakanotani, T. Higuchi, T. Furukawa et al., "High-efficiency organic light-emitting diodes with fluorescent emitters," Nat. Commun., vol. 5, pp. 4016, 2014.

[26] C. Poriel, and J. Rault-Berthelot, "Blue Single-Layer Organic Light-Emitting Diodes Using Fluorescent Materials: A Molecular Design View Point," Advanced Functional Materials, vol. 30, no. 17, pp. 1910040, 2020.

[27] T. Smith, and J. Guild, "The C.I.E. colorimetric standards and their use," Transactions of the Optical Society, vol. 33, no. 3, pp. 73-134, 1931/01/01, 1931.

[28] P. DESTRUEL, and P. JOLINAT, Technique de l'Ingénieur-Diodes électroluminescentes organiques (OLED), vol. 
[IN 25], 2004.

[29] C. FERY, and P. LE ROY, Technique de l'Ingénieur-Affichage-Visualisation-Ecrans OLED, vol. [R 620], 2005.

[30] P. LE BARNY, Technique de l'Ingénieur-Electroluminescences des matériaux organiques. Principes de base, vol. [E 3 106], 2006.

[31] P. LE BARNY, Technique de l'Ingénieur-Electroluminescences des matériaux organiques. Technologies, vol. [N 407], 2006.

[32] P. JOLINAT, Technique de l'Ingénieur- Diodes électroluminescentes organiques (OLED) - Technologies, vol. [E 6 505], 2011.

[33] P. JOLINAT, C. RENAUD, and M. TERNISIEN, "Diodes électroluminescentes organiques Technologie," Techniques de l'Ingénieur-Diodes électroluminescentes organiques - Technologie, vol. base documentaire : TIB451DUO, no. ref. article : e6505, 2016.

[34] N. SEBAIHI, J. CORNIL, and P. VIVILLE, "Diodes électroluminescentes organiques (OLED) émettrices de lumière blanche-Caractéristiques et applications pour l'éclairage.," Technique de l'Ingénieur-Diodes électroluminescentes organiques (OLED) émettrices de lumière blanche-Caractéristiques et applications pour l'éclairage., vol. [IN 145], 2011.

[35] S. FORGET, and S. CHESNAIS, Technique de l'Ingénieur-Matériaux organiques pour diodes électroluminescentes et lasers vol. E 6 435, pp. 1-17, 2014.

[36] C. PORIEL, and J. RAULT-BERTHELOT, "Un design moléculaire unique pour des OLED à excimères à base d'oligophénylènes," Technique de l'Ingénieur-Un design moléculaire unique pour des OLED à excimères à base d'oligophénylènes, vol. [IN 163], 2013.

[37] C. PORIEL, and J. RAULT-BERTHELOT, "Instabilité de la couleur d'émission des OLEDs bleues à base d'oligophénylènes pontés" Technique de l'Ingénieur-Instabilité de la couleur d'émission des OLEDs bleues à base d'oligophénylènes pontés vol. IN 169, pp. 1-17, 2014.

[38] C. PORIEL, and J. RAULT-BERTHELOT, "Molécules organiques fluorescentes comme matériau actif d'OLED bleues monocouches," Technique de l'Ingénieur-Molécules organiques fluorescentes comme matériau actif d'OLED bleues monocouches, vol. IN 168, pp. 1-21, 2015.

[39] OLED Fundamentals: Materials, Devices, and Processing of Organic Light-Emitting Diodes: CRC press, 2015.

[40] Organic and Printed Electronics: Fundamentals and Applications: Pan Stanford 2016.

[41] H. Yersin, Highly Efficient OLEDs with Phosphorescent Materials, Universitaet Regensburg, Germany: Wiley-VCH, Berlin, 2007.

[42] J. Brown, R. Kwong, Y.-J. Tung et al., "Recent progress in high-efficiency phosphorescent OLED technology," $J$. Soc. Inf. Disp., vol. 12, no. 3, pp. 329-332, 2004.

[43] H. Uoyama, K. Goushi, K. Shizu et al., "Highly efficient organic light-emitting diodes from delayed fluorescence," Nature, vol. 492, pp. 2342012.

[44] J. Miller, "Fluorescent molecules offer another route to efficient organic LEDs," Physicstoday, vol. 66, no. 2, pp. 10, 2013.

[45] W. Song, and K. S. Yook, "Hyperfluorescence-based full fluorescent white organic light-emitting diodes," J. Ind. Eng. Chem. , vol. 61, pp. 445-448, 2018/05/25/, 2018.

[46] C. Adachi, M. A. Baldo, M. E. Thompson et al., "Nearly 100\% internal phosphorescence efficiency in an organic emitting device," J. Appl. Phys., vol. 90, no. 10, pp. 5048-5051, 2001.

[47] S.-Y. Kim, and J.-J. Kim, "Outcoupling efficiency of organic light emitting diodes and the effect of ITO thickness," Org. Electron., vol. 11, no. 6, pp. 1010-1015, 2010.

[48] X.-L. Li, M. Liu, Y. Li et al., "Structure-simplified and highly efficient deep blue organic light-emitting diodes with reduced efficiency roll-off at extremely high luminance," Chem. Commun., vol. 52, no. 100, pp. 14454-14457, 2016.

[49] D. Yokoyama, Y. Park, B. Kim et al., "Dual efficiency enhancement by delayed fluorescence and dipole orientation in high-efficiency fluorescent organic light-emitting diodes," Appl. Phys. Lett., vol. 99, no. 12, pp. 123303, 2011.

[50] S.-K. Kim, B. Yang, Y. Ma et al., "Exceedingly efficient deep-blue electroluminescence from new anthracenes obtained using rational molecular design," J. Mater. Chem., vol. 18, no. 28, pp. $3376-3384,2008$.

[51] W.-C. Chen, Y. Yuan, S.-F. Ni et al., "Highly Efficient Deep-Blue Electroluminescence from a Charge-Transfer Emitter with Stable Donor Skeleton," ACS Appl. Mater. Interfaces vol. 9, pp. 7331-7338, 2017/02/10, 2017.

[52] L. Duan, J. Qiao, Y. Sun et al., "Ambipolar Transporting 1,2-Benzanthracene Derivative with Efficient Green Excimer Emission for Single-Layer Organic Light-Emitting Diodes,” Adv. Optical Mater., vol. 1, no. 2, pp. 167-172, 2013.

[53] T.-H. Huang, J. T. Lin, L.-Y. Chen et al., "Dipolar dibenzothiophene S,S-dioxide derivatives containing diarylamine: Materials for single-layer organic ligth-emitting devices," Adv. Mater., vol. 18, pp. 602-606, 2006.

[54] M. Cai, D. Zhang, T. Huang et al., "Multifunctional Materials for High-Performance Double-Layer Organic LightEmitting Diodes: Comparison of Isomers with and without Thermally Activated Delayed Fluorescence," ACS Applied Materials \& Interfaces, vol. 9, no. 20, pp. 17279-17289, 2017/05/24, 2017.

[55] Z. Zhao, J. Geng, Z. Chang et al., "A tetraphenylethene-based red luminophor for an efficient non-doped electroluminescence device and cellular imaging," J. Mater. Chem., vol. 22, no. 22, pp. 11018, 2012.

[56] Q. Wang, F. Lucas, C. Quinton et al., "Evolution of pure hydrocarbon hosts: simpler structure, higher performance and universal application in RGB phosphorescent organic light-emitting diodes," Chemical Science, vol. 11, no. 19, pp. 4887-4894, 2020

[57] L. J. Sicard, H.-C. Li, Q. Wang et al., "C1-Linked Spirobifluorene Dimers: Pure Hydrocarbon Hosts for HighPerformance Blue Phosphorescent OLEDs," Angew. Chem., Int. Ed., vol. 58, no. 12, pp. 3848-3853, 2019.

[58] C. Poriel, J. Rault-Berthelot, and L. J. Sicard, "New Generations of Spirobifluorene Regioisomers for Organic Electronics: Tuning Electronic Properties with the Substitution Pattern," Chemical Communications, vol. 55, pp. 14238-14254, 2019.

[59] C. Poriel, and J. Rault-Berthelot, "Structure-property relationship of 4-substituted-spirobifluorenes as hosts for phosphorescent organic light emitting diodes: an overview," Journal of Materials Chemistry C, vol. 5, no. 16, pp. $3869-3897,2017$ 

51, no. 8, pp. 1818-1830, 2018/08/21, 2018.

[61] J.-H. Lee, C.-H. Chen, P.-H. Lee et al., "Blue organic light-emitting diodes: current status, challenges, and future outlook," J. Mater. Chem. C, vol. 7, no. 20, pp. 5874-5888, 2019.

[62] Y. Im, S. Y. Byun, J. H. Kim et al., "Recent Progress in High-Efficiency Blue-Light-Emitting Materials for Organic Light-Emitting Diodes," Adv. Funct. Mater., vol. 27, no. 13, pp. 1603007, 2017.

[63] X. Yang, X. Xu, and G. Zhou, "Recent advances of the emitters for high performance deep-blue organic lightemitting diodes," J. Mater. Chem. C, vol. 3, pp. 913-944, 2015.

[64] F.-M. Hsu, L.-J. Chien, K.-T. Chen et al., "High morphology stability and ambipolar transporting host for use in blue phosphorescent single-layer organic light-emitting diodes," Org. Electron., vol. 15, pp. 3327-3332, 2014.

[65] F. Lucas, O. A. Ibraikulov, C. Quinton et al., "Spirophenylacridine-2,7-(diphenylphosphineoxide)-fluorene: A Bipolar Host for High-Efficiency Single-Layer Blue Phosphorescent Organic Light-Emitting Diodes," Advanced Optical Materials, vol. 8, no. 2, pp. 1901225, 2020.

[66] W.-C. Chen, Y. Yuan, Z.-L. Zhu et al., "De novo design of D- $\sigma-A$ molecules as universal hosts for monochrome and white phosphorescent organic light-emitting diodes," Chemical Science, vol. 9, no. 17, pp. 4062-4070, 2018.

[67] J. H. Kim, S.-H. Hwang, W. Song et al., "Acridine modified dibenzothiophene derivatives as high triplet energy host materials for blue phosphorescent organic light-emitting diodes," Dyes Pigm., vol. 122, pp. 103-108, 2015/11/01/, 2015 .

[68] C. W. Lee, and J. Y. Lee, “Above 30\% External Quantum Efficiency in Blue Phosphorescent Organic Light-Emitting Diodes Using Pyrido[2,3-b]indole Derivatives as Host Materials," Adv. Mater., vol. 25, no. 38, pp. 5450-5454, 2013.

[69] A. Maheshwaran, V. G. Sree, H.-Y. Park et al., "High Efficiency Deep-Blue Phosphorescent Organic Light-Emitting Diodes with CIE x, y $(\leq 0.15)$ and Low Efficiency Roll-Off by Employing a High Triplet Energy Bipolar Host Material," Adv. Funct. Mater., vol. 28, no. 36, pp. 1802945, 2018.

[70] C. Zang, X. Peng, H. Wang et al., "Efficient multilayer and single layer phosphorescent organic light-emitting devices using a host with balanced bipolar transporting properties and appropriate energy level," Org. Electron., vol. 50, pp. 106-114, 2017/11/01/, 2017.

[71] S. Thiery, D. Tondelier, B. Geffroy et al., "Spirobifluorene-2,7-dicarbazole-4'-phosphine Oxide as Host for HighPerformance Single-Layer Green Phosphorescent OLED Devices," Org. Lett., vol. 17, no. 19, pp. 4682-4685, 2015/10/02, 2015 .

[72] X. Tang, X.-Y. Liu, Y. Yuan et al., "High-Efficiency White Organic Light-Emitting Diodes Integrating Gradient Exciplex Allocation System and Novel D-Spiro-A Materials," ACS Appl. Mater. Interfaces, vol. 10, no. 35, pp. 29840-29847, 2018/09/05, 2018.

[73] M. Kim, and J. Y. Lee, "Engineering of Interconnect Position of Bicarbazole for High External Quantum Efficiency in Green and Blue Phosphorescent Organic Light-Emitting Diodes," ACS Applied Materials \& Interfaces, vol. 6, no. 17, pp. 14874-14880, 2014/09/10, 2014.

[74] S. Thiery, C. Declairieux, D. Tondelier et al., "2-substituted vs 4-substituted-9,9'-spirobifluorene host materials for green and blue phosphorescent OLEDs: a structure property relationship study," Tetrahedron, vol. 70, pp. 63376351, 2014.

[75] S. Thiery, B. Heinrich, B. Donnio et al., "Modulation of the Electronic and Mesomorphic Properties of AlkynylSpirobifluorene Compounds as a Function of the Substitution Pattern," J. Phys. Chem. C, vol. 119, no. 19, pp. 1056410575, //, 2015.

[76] C. Quinton, S. Thiery, O. Jeannin et al., "Electron-Rich 4-Substituted Spirobifluorenes: Toward a New Family of High Triplet Energy Host Materials for High-Efficiency Green and Sky Blue Phosphorescent OLEDs," ACS Appl. Mater. Interfaces, vol. 9, no. 7, pp. 6194-6206, 2017/02/22, 2017.

[77] M. Romain, D. Tondelier, O. Jeannin et al., "Properties modulation of organic semi-conductors based on a DonorSpiro-Acceptor (D-spiro-A) molecular design: New host materials for efficient sky-blue PhOLEDs," J. Mater. Chem. C, vol. 3, pp. 9701-9714, 2015.

[78] M. Romain, D. Tondelier, B. Geffroy et al., "Spiro-configured phenyl acridine thioxanthene dioxide as host for efficient PhOLEDs," Chem. Commun., vol. 51, no. 7, pp. 1313-1315, 2015.

[79] S. Thiery, D. Tondelier, B. Geffroy et al., "Modulation of the physicochemical properties of Donor-Spiro-Acceptor derivatives through the planarization of their Donor unit: phenylacridine vs indoloacridine. New Indoloacridinespiro-acceptor hosts for efficient green and sky-blue PhOLEDs," Chem. Eur. J., vol. 22, pp. 10136-10149, 2016.

[80] C. Poriel, J. Rault-Berthelot, S. Thiery et al., "9H-Quinolino[3,2,1-k]phenothiazine: A New Electron-Rich Fragment for Organic Electronics," Chem. Eur. J., vol. 22, no. 50, pp. 17930-17935, 2016.

[81] M.-Y. Lai, C.-H. Chen, W.-S. Huang et al., "Benzimidazole/Amine-Based Compounds Capable of Ambipolar Transport for Application in Single-Layer Blue-Emitting OLEDs and as Hosts for Phosphorescent Emitters," Angew. Chem. Int. Ed., vol. 47, no. 3, pp. 581-585, 2008.

[82] Z. Xu, J. Gu, X. Qiao et al., "Highly Efficient Deep Blue Aggregation-Induced Emission Organic Molecule: A Promising Multifunctional Electroluminescence Material for Blue/Green/Orange/Red/White OLEDs with Superior Efficiency and Low Roll-Off," ACS Photonics, vol. 6, no. 3, pp. 767-778, 2019/03/20, 2019.

[83] K. Gao, K. Liu, X.-L. Li et al., "An ideal universal host for highly efficient full-color, white phosphorescent and TADF OLEDs with a simple and unified structure," J. Mater. Chem. C, vol. 5, no. 39, pp. 10406-10416, 2017.

[84] C. Cao, W.-C. Chen, J.-X. Chen et al., "Bipolar Blue Host Emitter with Unity Quantum Yield Allows Full Exciton Radiation in Single-Emissive-Layer Hybrid White Organic Light-Emitting Diodes," ACS Applied Materials \& Interfaces, vol. 11, no. 12, pp. 11691-11698, 2019/03/27, 2019.

[85] X.-Y. Liu, X. Tang, Y. Zhao et al., "Dispiro and Propellane: Novel Molecular Platforms for Highly Efficient Organic Light-Emitting Diodes," ACS Appl. Mater. Interfaces, vol. 10, no. 2, pp. 1925-1932, 2018/01/17, 2018.

[86] Q. Ran, Y.-L. Zhang, X. Hua et al., "Modulation of p-type units in tripodal bipolar hosts towards highly efficient red phosphorescent OLEDs,” Dyes Pigm., vol. 162, pp. 632-639, 2019/03/01/, 2019.

[87] L. Ding, S.-C. Dong, Z.-Q. Jiang et al., "Orthogonal Molecular Structure for Better Host Material in Blue Phosphorescence and Larger OLED White Lighting Panel," Adv. Funct. Mater., vol. 25, no. 4, pp. 645-650, 2015. 
[88] M. Godumala, S. Choi, M. J. Cho et al., "Recent breakthroughs in thermally activated delayed fluorescence organic light emitting diodes containing non-doped emitting layers," Journal of Materials Chemistry C, vol. 7, no. 8, pp. 2172-2198, 2019.

[89] M. Y. Wong, and E. Zysman-Colman, "Purely Organic Thermally Activated Delayed Fluorescence Materials for Organic Light-Emitting Diodes," Adv. Mater., vol. 29, no. 22, pp. 1605444, 2017/06/01, 2017.

[90] Y. Im, M. Kim, Y. J. Cho et al., "Molecular Design Strategy of Organic Thermally Activated Delayed Fluorescence Emitters," Chem. Mater., vol. 29, no. 5, pp. 1946-1963, 2017/03/14, 2017.

[91] T. Chatterjee, and K.-T. Wong, "Perspective on Host Materials for Thermally Activated Delayed Fluorescence Organic Light Emitting Diodes," Adv. Optical Mater., vol. 7, no. 1, pp. 1800565, 2019.

[92] Q. Zhang, D. Tsang, H. Kuwabara et al., "Nearly 100\% Internal Quantum Efficiency in Undoped Electroluminescent Devices Employing Pure Organic Emitters," Adv. Mater., vol. 27, no. 12, pp. 2096-2100, 2015.

[93] T.-A. Lin, T. Chatterjee, W.-L. Tsai et al., "Sky-Blue Organic Light Emitting Diode with 37\% External Quantum Efficiency Using Thermally Activated Delayed Fluorescence from Spiroacridine-Triazine Hybrid," Adv. Mater., vol. 28, no. 32, pp. 6976-6983, 2016.

[94] P. Rajamalli, N. Senthilkumar, P. Y. Huang et al., "New Molecular Design Concurrently Providing Superior Pure Blue, Thermally Activated Delayed Fluorescence and Optical Out-Coupling Efficiencies," J. Am. Chem. Soc., vol. 139, no. 32, pp. 10948-10951, 2017/08/16, 2017.

[95] B. Li, Z. Li, T. Hu et al., "Highly efficient blue organic light-emitting diodes from pyrimidine-based thermally activated delayed fluorescence emitters," J. Mater. Chem. C, vol. 6, no. 9, pp. 2351-2359, 2018.

[96] M.-S. Lin, S.-J. Yang, H.-W. Chang et al., "Incorporation of a CN group into mCP: a new bipolar host material for highly efficient blue and white electrophosphorescent devices," J. Mater. Chem. C, vol. 22, no. 31, pp. 16114-16120, 2012.

[97] C. Han, Y. Zhao, H. Xu et al., "A Simple Phosphine-Oxide Host with a Multi-insulating Structure: High Triplet Energy Level for Efficient Blue Electrophosphorescence," Chemistry - A European Journal, vol. 17, no. 21, pp. 5800-5803, 2011.

[98] T.-L. Wu, M.-J. Huang, C.-C. Lin et al., "Diboron compound-based organic light-emitting diodes with high efficiency and reduced efficiency roll-off," Nature Photonics, vol. 12, no. 4, pp. 235-240, 2018/04/01, 2018.

[99] M. Liu, R. Komatsu, X. Cai et al., "Horizontally Orientated Sticklike Emitters: Enhancement of Intrinsic OutCoupling Factor and Electroluminescence Performance," Chem. Mater., vol. 29, no. 20, pp. 8630-8636, 2017/10/24, 2017.

[100] N. B. Kotadiya, P. W. M. Blom, and G.-J. A. H. Wetzelaer, "Efficient and stable single-layer organic light-emitting diodes based on thermally activated delayed fluorescence," Nature Photonics, 2019/07/08, 2019.

[101] W. Zeng, H.-Y. Lai, W.-K. Lee et al., "Achieving Nearly 30\% External Quantum Efficiency for Orange-Red Organic Light Emitting Diodes by Employing Thermally Activated Delayed Fluorescence Emitters Composed of 1,8Naphthalimide-Acridine Hybrids," Adv. Mater., vol. 30, no. 5, pp. 1704961, 2018.

[102] L. Yu, Z. Wu, G. Xie et al., "An efficient exciton harvest route for high-performance OLEDs based on aggregationinduced delayed fluorescence," Chem. Commun., vol. 54, no. 11, pp. 1379-1382, 2018.

[103] J. Xue, Q. Liang, R. Wang et al., "Highly Efficient Thermally Activated Delayed Fluorescence via J-Aggregates with Strong Intermolecular Charge Transfer," Adv. Mater., vol. 31, no. 28, pp. 1808242, 2019.

[104] Y.-L. Zhang, Q. Ran, Q. Wang et al., "High-Efficiency Red Organic Light-Emitting Diodes with External Quantum Efficiency Close to $30 \%$ Based on a Novel Thermally Activated Delayed Fluorescence Emitter," Advanced Materials, vol. 31, no. 42, pp. 1902368, 2019.

[105] Y. J. Cho, K. S. Yook, and J. Y. Lee, "Cool and warm hybrid white organic light-emitting diode with blue delayed fluorescent emitter both as blue emitter and triplet host," Scientific Reports, vol. 5, pp. 7859, 01/19/online, 2015.

[106] Y. Li, X.-L. Li, D. Chen et al., "Design Strategy of Blue and Yellow Thermally Activated Delayed Fluorescence Emitters and Their All-Fluorescence White OLEDs with External Quantum Efficiency beyond 20\%," Advanced Functional Materials, vol. 26, no. 38, pp. 6904-6912, 2016.

[107] J. Zhao, X. Chen, Z. Yang et al., "Highly-efficient fully non-doped white organic light-emitting diodes consisting entirely of thermally activated delayed fluorescence emitters," Journal of Materials Chemistry C, vol. 6, no. 13, pp. 3226-3232, 2018.

[108] Y. Liu, C. Li, Z. Ren et al., "All-organic thermally activated delayed fluorescence materials for organic light-emitting diodes," Nature Reviews Materials, vol. 3, pp. 18020, 04/10/online, 2018.

[109] J. H. Kim, Yun, J. H., \& Lee, J. Y. ().. , ., "Recent Progress of Highly Efficient Red and Near-Infrared Thermally Activated Delayed Fluorescent Emitters," Advanced Optical Materials, vol. 6, pp. 1800255, 2018.

[110] "Cynora," https://www.cynora.com/technology/oled/.

[111] Decomposition temperature Td is defined as the temperature at which 5\% loss occurs during heating

[112] "Toray et idemitsu Kosan," https://cs2.toray.co.jp/news/toray/en/newsrrs02.nsf/0/565D35374C2D7986492581A7000FD59A.

[113] "Kyulux," https://www.kyulux.com/our-technology/.

[114] C. Adachi, "Third generation OLED by hyperfluorescence," Digest of Technical Papers - SID International Symposium, vol. 44, no. 1, pp. 513-514, 2013.

[115] H. Yersin, L. Mataranga-Popa, S.-W. Li et al., "Design strategies for materials showing thermally activated delayed fluorescence and beyond: Towards the fourth-generation OLED mechanism," J SOC INF DISPLAY, vol. 26, no. 4, pp. 194-199, 2018/04/01, 2018.

[116] H.-F. Chen, S.-J. Yang, Z.-H. Tsai et al., "1,3,5-Triazine derivatives as new electron transport-type host materials for highly efficient green phosphorescent OLEDs," Journal of Materials Chemistry, vol. 19, no. 43, pp. 8112-8118, 2009.

[117] "HyperOLED Projet Européen," https://www.electronique-eci.com/news/financement-europeen-de-4-millionsdeuros-pour-les-oled-hyperfluorescents. 


\section{Normes et standards}

ISO Acoustique : méthode de calcul du niveau d'isotonie ISO 5321975 ISO, 1975.

AFNOR Tuiles en terre cuite pour posé en discontinu. Détermination des caractéristiques physiques. Partie 1 : essai d'imperméabilité NF EN 539-1 AFNOR, 1994.

\section{Réglementation}

Loi n 83-634 du 13 juillet 1983 portant droits et obligations... (version consolidée du 3 mars 2002).

Décret $\mathrm{n}^{\circ}$ 2000-44 du 13 janvier 2000 portant... (version consolidée au 5 octobre 2007) JO $\mathrm{n}^{\circ} 11 \mathrm{du} 14$ janvier 2000 page 369 NOR : FPPA9910013D.

\section{Brevets}

Procédé d'oxydation électrolytique du cérium et ensemble d'électrolyse pour sa mise en œuvre CA2582058

\section{Annuaire}

\section{Constructeurs - Fournisseurs - Distributeurs (liste non exhaustive)}

Avec adresse Internet

Presses à injecter horizontales électriques

Arburg SAS Hawaï distribué par Arburg France http://www.arburg.fr

Organismes - Fédérations - Associations (liste non exhaustive)

Avec adresse Internet

Organisme professionnel de prévention du bâtiment et des travaux publics OPPBT http://www.oppbt.fr

\section{Documentation - Formation - Séminaires (liste non exhaustive)}

Avec adresse Internet

Séminaire MCBT. Films supraconducteurs à HTc et THTc: rôle des interfaces dans leur élaboration par voie chimique. Philippe Odier mardi 20 novembre 2007 Institut Néel http://neel.cnrs.fr/spip.php?article1324

Laboratoires - Bureaux d'études - Écoles - Centres de recherche (liste non exhaustive)

Avec adresse Internet

Centre technique et scientifique du bâtiment CSTB http//www.cstb.fr 